\title{
Mobility of Hydrogen-Vacancy Complexes in Nickel
}

\author{
by

\begin{abstract}
Bopanna Kokkengada Madappa
\end{abstract}
\author{
A thesis submitted to the \\ Faculty of Graduate and Postdoctoral Affairs \\ in partial fulfillment of the requirements for the degree of
}

Master of Applied Science in Mechanical Engineering

Ottawa-Carleton Institute for Mechanical and Aerospace Engineering

Department of Mechanical and Aerospace Engineering

\author{
Carleton University \\ Ottawa, Ontario
}

August, 2016

(c) Copyright

Bopanna Kokkengada Madappa, 2016 
The undersigned hereby recommends to the

Faculty of Graduate and Postdoctoral Affairs acceptance of the thesis

\title{
Mobility of Hydrogen-Vacancy Complexes in Nickel
}

\author{
submitted by Bopanna Kokkengada Madappa \\ in partial fulfillment of the requirements for the degree of
}

Master of Applied Science in Mechanical Engineering

Dr. Ronald E.Miller, Supervisor

Dr. Ronald E.Miller, Department Chair

Carleton University

August, 2016 


\section{Abstract}

Atomistic simulations of the mechanism of hydrogen pile-up around a single vacancy and its effect on the vacancy mobility are performed to investigate possible nanoscale mechanisms for hydrogen embrittlement. A detailed study of the interactions of hydrogen with the vacancy point defect in nickel is presented. In particular we discuss the trapping of multiple hydrogen atoms in a monovacancy. We show that a single vacancy is capable of trapping 12 or more hydrogen atoms. The trapping energy is found to be approximately equal to $-0.14 \mathrm{eV}$. We find that the interstitial hydrogen in the bulk lattice prefers to be located at octahedral interstitial positions which is in agreement with previous theoretical works in the literature. The trapping energy is obtained for the relaxed octahedral and tetrahedral positions inside the vacancy, with a site preference for octahedral positions. The influence of the vacancy defect is not limited to its volume but also extends to the octahedral sites of its first-nearestneighbour atoms. The interstitial hydrogen atoms prefer to cluster around the single vacancy than to remain at unrelated interstitial locations in the bulk lattice. The hydrogen-vacancy cluster is extremely stable in nature.

We also look at the effect of pressure on the trapping and migration energies of the hydrogen-vacancy complex. We find that the magnitude of the energy is influenced by stress. The hydrogen atom cloud around the vacancy defect also exerts some resistance towards the migration of the defect. 


\section{Acknowledgments}

I take this opportunity to express my sincere gratitude to my supervisor, Professor Ronald E. Miller whose vision, guidance and encouragement aided me to successfully undertake and complete this research assignment. He introduced me to the world of computational material science and was always there to listen to the hurdles I faced. His advice and ideas to tackle problems were indispensable without which this project would not have been possible. In addition, I would like to thank the thesis review committee for their time and effort.

I would like to share this moment of happiness with my parents and thank them for their constant love, encouragement and support. My parent's faith and sacrifices over the years have always kept me moving forward towards my goals.

I am thankful to Dr. Jean-François Joly from my research group for his time and valuable advice during my research tenure. In addition I would like to express my appreciation to my research group mate Ms. Roxana Barceló Singh for her thoughts, ideas and valuable discussions. I also gratefully acknowledge the cooperation that I received from other faculty members and administrative staffs of my Department. Lastly I would like to recognize the Department of Mechanical and Aerospace Engi-

neering and the Faculty of Graduate Studies and Research of Carleton University for giving me the opportunity and facility for pursuing my masters program. 
To my mother 


\section{Table of Contents}

Abstract $\quad$ iii

Acknowledgments $\quad$ iv

Table of Contents vi

List of Tables $\quad$ ix

List of Figures $\quad$ X

Nomenclature $\quad$ xiv

1 Introduction 1

1.1 Material Science and Crystals . . . . . . . . . . . . . . 1

1.2 Defects in Crystals ..................... 5

1.3 Nickel Metal . . . . . . . . . . . . . . . . . . 8

1.3.1 Interstitial Sites in Close Packed Lattices . . . . . . . . . . . . 10

1.4 Hydrogen in Metals . . . . . . . . . . . . . . . . . . . . . . 12

1.4.1 Introduction to Hydrogen Embrittlement . . . . . . . . . . . . 12

1.5 Atomistic Modeling and Scheme . . . . . . . . . . . . . . . 16

1.6 Motivation for this research . . . . . . . . . . . . . . 18

1.7 Thesis outline . . . . . . . . . . . . . . . . . . . . 21 
2.1 Features of the Potential Energy Landscape . . . . . . . . . 27

2.2 Relevance of Locating Minima . . . . . . . . . . . . . . . . . 28

2.3 Identifying the Local Energy Minima . . . . . . . . . . . . . . 29

2.3 .1 Boundaries . . . . . . . . . . . . . . . . . 30

2.3.2 Steepest Descent Method . . . . . . . . . . . . . . . . . . 32

2.3.3 Selection of Energy Minimization Method . . . . . . . . 33

2.4 Application to Crystals and Defects . . . . . . . . . . . . 34

2.4.1 Cohesive Energy of an Infinite Crystal _ . . . . . . . . 35

2.4.2 Crystal Defects - Vacancies . . . . . . . . . . . . 36

2.4 .3 Crystal Defects - Interstitials _ . . . . . . . . . . 37

3 Background Material and Literature Review 39

3.1 Diffusion of Hydrogen in Metals . . . . . . . . . . . . . . . 40

3.2 Hydrogen-Metal interactions . . . . . . . . . . . . . . . . . 42

3.2.1 Hydrogen-Point Defect Interactions _ . . . . . . . . . . 43

$3.2 .2 \quad$ Hydrogen Embrittlement . . . . . . . . . . . . . . 48

3.3 Theories for Hydrogen Trapping . . . . . . . . . . . . . . . . 51

3.4 Activation Energy for Vacancy Migration . . . . . . . . . . . 57

4 Methodology $\quad 60$

4.1 Simulation Setup and Design . . . . . . . . . . . . . . 61

4.1 .1 Interatomic Potential . . . . . . . . . . . . . . . 61

4.1.2 Simulation Technique and Methology . . . . . . . . . . . 63

4.1 .3 Preliminary Calculations . . . . . . . . . . . . . 65

4.1.4 Investigation of the Hydrogen-Vacancy Cluster . . . . . . . 70

4.1.5 Hydrogen-Vacancy Complex Migration . . . . . . . . . . 72 
$\begin{array}{lll}5 & \text { Results and Analysis } & 74\end{array}$

5.1 Verification of the Bulk Properties of Defect Free Nickel . . . . . . . . 74

5.2 Interactions between Hydrogen and a Monovacancy . . . . . . . . . . 76

5.3 Trapping Effect of Multiple Hydrogen Interstitials at a Monovacancy 78

5.3.1 Hydrogen-Vacancy Energetics . . . . . . . . . . . . . 78

5.3.2 Hydrogen-Vacancy Energetics involving First-NearestNeighbor Sites . . . . . . . . . . . . . . . . 79

5.3.3 Interstitial Hydrogen Complex Energetics . . . . . . . . . . . . 82

5.3.4 Maximum Trapping Capacity of a Monovacancy . . . . . . . . 83

5.3.5 Hydrogen-Vacancy Interaction based on Simulation Cell Size . 85

5.4 Effect of Pressure on the Hydrogen-Vacancy Complex . . . . . . . . . 87

5.4.1 Activation Energy for Vacancy Migration . . . . . . . . . . . . 87

5.4.2 Trapping Energy . . . . . . . . . . . . . . . . . . . . . 90

6 Conclusions and Future Recommendations $\quad 92$

$\begin{array}{ll}\text { List of References } & 95\end{array}$

Appendix A Hydrogen trapping energy as a function of system size 107 


\section{List of Tables}

4.1 Comparison of the calculated values of nickel from the EAM potential with the experimental values (Table reproduced from [1]) . . . . . 63

4.2 Hydrogen interstitial formation energy . . . . . . . . . . . . 68

5.1 The properties of bulk nickel: lattice parameter and cohesive energy

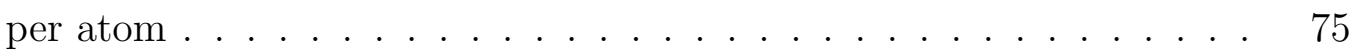

5.2 Hydrogen trapping energy as per respective configurations . . . . . 77 


\section{List of Figures}

1.1 Illustration of the FCC lattice arrangement in nickel . . . . . . . . . . 9

1.2 Illustration of tetrahedral interstitial sites in an FCC lattice . . . . . 11

1.3 Illustration of octahedral interstitial sites in an FCC lattice . . . . . . 11

1.4 Global portrayal of hydrogen embrittlement interaction . . . . . . . . 14

2.1 Schematic representation of a $3 N$-dimensional energy landscape (Figure reproduced by author from $[2])$. . . . . . . . . . . . . . . . 25

2.2 Movement of a atom in a $3 N$-dimensional potential energy landscape 26

2.3 Illustration of the periodic boundary condition in two dimensions. When the filled-in atoms exit one end of the simulation box, they enter in the opposite end and in this manner it mimics an infinite bulk structure. The center square is our simulation cell while the rest are images of this. . . . . . . . . . . . . . . . . 31

2.4 Illustration of the steepest descent method implemented on the PEL . 32

2.5 (a)Illustration of a defect free atomic arrangement; (b) Illustration of a atomic arrangement with one vacancy . . . . . . . . . . . .

2.6 (a)Illustration of the atomic arrangement with a self-interstitial; (b) Illustration of the atomic arrangement with impurity interstitial atoms

3.1 Illustration of the mechanism of activation energy for diffusion of an interstitial hydrogen atom . . . . . . . . . . . . . 
3.2 Trapping energy per hydrogen atom (in $\mathrm{Al}$ ) in $\mathrm{eV}$ as a function of the number of hydrogen atoms trapped in a monovacancy (Image reproduced from $[3]) \ldots \ldots \ldots \ldots \ldots \ldots \ldots$

3.3 (a)Projected atomistic view of dislocation cores surrounded by clouds of hydrogen atoms; (b) Close proximity atomistic view of a dislocation core surrounded by hydrogen atoms. Figure reproduced from [4] . . .

4.1 Illustration of the formation of a single vacancy in a perfect lattice

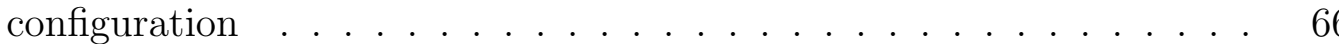

4.2 Figure (a) Schematic of a hydrogen interstitial in perfect 2D lattice; Figure (b) Hydrogen interstitial at the octahedral (O) and tetrahedral (T) sites in an FCC lattice . . . . . . . . . . . . . . . .

4.3 Figure (a) represents the cell with vacancy and interstitial defects unrelated each other; Figure (b) represents the trapped hydrogen interstitial at a vacancy . . . . . . . . . . . . . . . . .

4.4 Hydrogen-vacancy (V) configurations at the substitution (S), octahedral $(\mathrm{O})$ and tetrahedral $(\mathrm{T})$ sites in an FCC lattice . . . . . . . . . 70

4.5 Schematic representation of a hydrogen-vacancy cluster . . . . . . . 71

5.1 The total energy curve of nickel as a function of atomic volume . . . 76

5.2 Trapping energy per hydrogen atom in $\mathrm{eV}$ as a function of the number of hydrogen atoms being trapped at the octahedral and tetrahedral sites of a vacancy . . . . . . . . . . . . . . .

5.3 Trapping energy per hydrogen atom in $\mathrm{eV}$ as a function of the number of hydrogen atoms being trapped at the octahedral sites . . . . . .

5.4 Schematic representation of hydrogen interstitials around a vacancy (V). The figure illustrates 12 hydrogen atoms at octahedral (O) sites around the vacancy and first-nearest-neighbor $\mathrm{Ni}$ atom $\mathrm{A} \quad \ldots .$. . 
5.5 Schematic representation of hydrogen interstitials around a vacancy (V). The figure illustrates 12 hydrogen atoms at octahedral (O) sites around the vacancy and first-nearest-neighbour Ni atom B . . . . .

5.6 Trapping energy per hydrogen atom in $\mathrm{eV}$ as a function of the number of hydrogen atoms being trapped (in the vicinity of a $\mathrm{Ni}$ atom and a

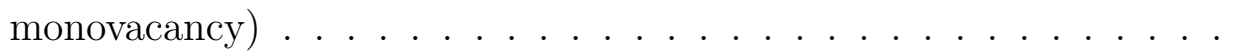

5.7 Trapping energy per hydrogen atom in $\mathrm{eV}$ as a function of the number of hydrogen atoms (exceeding 12) being trapped in and around a vacancy 84

5.8 Summary of trapping energy as a function of a trapped hydrogen atoms for a range of cell sizes . . . . . . . . . . . . . . . . . . 86

5.9 Illustration of activation energy for vacancy migration under the infuence of a hydrogen cloud . . . . . . . . . . . . . . .

5.10 Comparison of activation energy for vacancy migration as function of pressure for a hydrogen-vacancy complex and a single vacancy . . . .

5.11 Comparison of trapping energy of a vacancy as a function of pressure for a hydrogen-vacancy complex . . . . . . . . . . . . . .

A.1 The trapping energy of 1 hydrogen atom as a function of system size 108

A.2 The trapping energy of 2 hydrogen atoms as a function of system size 109

A.3 The trapping energy of 3 hydrogen atoms as a function of system size 110

A.4 The trapping energy of 4 hydrogen atoms as a function of system size 111

A.5 The trapping energy of 5 hydrogen atoms as a function of system size 112

A.6 The trapping energy of 6 hydrogen atoms as a function of system size 113

A.7 The trapping energy of 7 hydrogen atoms as a function of system size 114

A.8 The trapping energy of 8 hydrogen atoms as a function of system size 115

A.9 The trapping energy of 9 hydrogen atoms as a function of system size 116

A.10 The trapping energy of 10 hydrogen atoms as a function of system size 117 
A.11 The trapping energy of 11 hydrogen atoms as a function of system size 118 A.12 The trapping energy of 12 hydrogen atoms as a function of system size 119 


\section{Nomenclature}

\begin{tabular}{ll} 
FCC & Face-centered cubic \\
BCC & Body-centered cubic \\
HCP & Hexagonal close-packed \\
$k_{B}$ & Boltzmann constant \\
Ni & Nickel \\
a & Lattice constant \\
$\mathrm{H}$ & Hydrogen \\
HE & Hydrogen embrittlement \\
DFT & Density functional theory \\
$\phi$ & Pair potential \\
$r_{i, j}$ & Interatomic distance between atoms \\
$\rho$ & Periodic boundary condition \\
PEL & Potential energy landscape \\
\hline
\end{tabular}

xiv 


\begin{tabular}{|c|c|}
\hline SD & Steepest descent \\
\hline MS & Molecular stactics \\
\hline FIRE & Fast inertial relaxation engine \\
\hline$E_{c o h}$ & Cohesive energy \\
\hline$E_{\text {cell }}$ & Energy of the simulation cell \\
\hline$E_{\text {vac }}$ & Vacancy formation energy \\
\hline$E_{i}$ & Interstitial formation energy \\
\hline$n_{\text {cell }}$ & Number of atoms in a simulation cell \\
\hline $\mathrm{Pd}$ & Palladium \\
\hline MH & Metal hydride \\
\hline SAV & Super abundant vacancy \\
\hline $\mathrm{Al}$ & Aluminium \\
\hline$\AA$ & Angstrom \\
\hline HEDE & Hydrogen enhanced decohesion \\
\hline HELP & Hydrogen enhanced local plasticity \\
\hline HESIV & Hydrogen enhanced strain induced vacancy \\
\hline $\mathrm{D}$ & Deuterium \\
\hline W & Tungsten \\
\hline
\end{tabular}




\begin{tabular}{|c|c|}
\hline $\mathrm{Zr}$ & Zirconium \\
\hline $\mathrm{Fe}$ & Iron \\
\hline $\mathrm{Si}$ & Silicon \\
\hline ART & Activation relaxation technique \\
\hline $\mathrm{k}-\mathrm{ART}$ & Kinetic-activation relaxation technique \\
\hline LAMMPS & Large-scale atomic / molecular massively parallel simulator \\
\hline MPI & Message passing interface \\
\hline LJ & Lennard-Jones \\
\hline$C_{11}, C_{12}, C_{44}$ & Elastic constants \\
\hline$E_{m i g}$ & Migration energy \\
\hline$E_{n v}^{f}$ & Formation energy of $\mathrm{n}$ vacancy \\
\hline$E_{i}[H]$ & Hydrogen interstitial formation energy \\
\hline $\mathrm{O}$ & Octahedral site \\
\hline $\mathrm{T}$ & Tetrahedral site \\
\hline$E_{\text {trap }}(H)$ & Trapping energy of the vacancy \\
\hline$C_{V}$ & Vacancy concentration \\
\hline$C_{H}$ & Hydrogen concentration \\
\hline EAM & Embedded atom method \\
\hline
\end{tabular}


Ni-Al-H Nickel-Aluminium-Hydrogen 


\section{Chapter 1}

\section{Introduction}

\subsection{Material Science and Crystals}

The evolution of mankind and the evolution of material science are impossible to disentangle. The gravity of this statement is such that phases of human history have been named after materials; for example the stone, bronze and iron ages. One of the earliest recognizable events in material science is the firing of the clay and sand in an open fire to make pottery. In a modern sense, materials science and engineering is a multidisciplinary effort with an aim to understand material properties and work towards the development of new materials capable of greater performance.

Materials make modern life possible — from the polymers in the chair that we sit on, the metal ballpoint pen that we use daily, and the concrete that made the building we live in or work in to the materials that make up the streets and highways and the cars we drive in. All these items are the products of material science and technology. In short material science is the study of "stuff"; which may be solid matter, inorganic and organic. A link between the thermodynamic properties relating to the atomic structure and macroscopically evident properties of a material were first observed by Willard Gibbs [5] [6] in the late $19^{\text {th }}$ century. The atomic structure of a material is 
based on factors such as the constituent atoms, the methods involved in processing the material, whether it was subjected to external conditions like irradiation.

One physical classification of materials based on their morphological structure is into crystalline and amorphous (glass) structures. This study focuses on crystalline structures. Crystalline solids are a three dimensional collection of individual atoms, ions, or whole molecules organized in repeating patterns, whereas in amorphous solids the atoms are not organized in a definite lattice pattern. In this sense "lattice" means a three-dimensional array of points, coinciding with atom positions, and possessing translationally invariant symmetry. Understanding the properties of crystalline structures, such as their structure, melting point, ductility, conductivity and defects provides valuable insight into their applications.

The law of rational intercepts was created in 1784 by Haüy [7], which states that the crystal design could be uniquely identified by reference to the crystal axes, their relative lengths and angle of inclination. These axes, once defined, mark out a unit cell that is the smallest unit to possess the symmetry of the crystal as a whole. This in turn led to the identification of the seven systems of crystal symmetry; monoclinic, triclinic, cubic, tetragonal, rhombohedral, hexagonal and orthorhombic. Three relatively simple crystal structures [8] are found in most common metals: face-centered cubic (FCC), body-centered cubic (BCC) and hexagonal close-packed (HCP).

The fundamental properties of crystalline solids include a characteristic geometrical shape; bounded by planes or faces that intersect at a particular angle. In a crystal, the atoms form a repeating, periodic array. The unit cell of a crystal is a piece of the crystal that repeats itself throughout the whole material. We can assume the unit cell as a three-dimensional stamp that can be used to create the entire crystal. An ideal crystal is an infinite structure formed by regularly repeating the unit cell on a space-filling lattice. The infinite space filling arrangement of atoms in a regular 
pattern is a lattice. This definition is the standard of perfection to which real crystals are compared; essentially describing a perfect crystal of infinite dimensions containing no defects of any kind. Of course real crystals always have defects, and often it is the interplay between these defects that give rise to important material properties. In this thesis we study one particular type of defect, the hydrogen-vacancy complex in FCC nickel.

Now that we have understood the very fact that a perfect crystal is an idealization, there is no such thing in nature. In real materials the atom arrangements do not follow perfect crystalline patterns, i.e. they always contain certain defects or imperfections. The importance of defects depends upon the material, type of defect, its extent and properties. Material properties such as density and elastic constants are proportional to the concentration of the defects.

Perhaps the simplest and most common defects are vacancies; wherein an atom is simply missing from the crystal lattice site and interstitials; where an extra atom is inserted between the lattice sites. Less well known are the vacancy-interstitial complexes, where these point defects combine into a cluster. For example the importance of self-interstitials in silicon and the agglomeration of point defects leading to formation of more complex defects have been proven [9]. Unearthing the science that leads to the formation of these complex defects and studying the behavior of the materials by creating the defects at the microscopic level enables us to foresee the larger aspect of macroscopic failures, advanced high strength and lightweight materials.

With a brief understanding that crystals are made up of atoms arranged in a specific format, it is important to note that the atomic arrangement and bonding play an essential role in determining the material properties. Hence by implementing a combined approach of macroscopic testing (e.g. compressive or tensile test), and microscopic modeling (atomistic simulations), one can strive to comprehend the 
behavior of materials from the first principles of atomic bonding. The goal is to understand the behavior by modeling each atom in the material by atomistic simulations. The integration of the behavior of the atoms aids in perceiving how the material actually undergoes deformation or phase changes etc. thereby providing a relationship between the microscopic scale to the macroscopic scale. Since the interaction between the atoms is the foundation of all material science, atomistic modeling using virtual computational experiments can enormously reduce the cost and accelerate the time scales of developing new and advanced materials. Today this is achieved by quantum mechanics based techniques, and with the implementation of effective interactions between atoms called the interatomic potentials. The 1970s were the early days of atomistic modeling wherein even the fastest computers could manage full quantum mechanical calculations on a few atoms found in a simple crystal unit cell or a molecule. For example the prediction of the electronic band structure of phases of Si and Ge [10] was an atomistic modeling milestone in that era.

Over the last few decades, there has been a sudden realization that the understanding of nano-scale behavior in materials is mandatory to envisage material failure, which in turn opens up a wide range of opportunities to engineer new design methods for materials from the bottom up. The increasing trend towards miniaturization is a factor behind the significance of including atomistic simulations. Atomistic models typically may contain an extremely large number of particles, even though the actual physical dimensions involved will be quite small. Predicting the accurate behavior of such particle systems under explicit consideration of the trajectory of each particle is only possible by numerical simulation and this may involves large computational resources; the reason being since the basic inputs for an atomistic simulation are the atomic positions, velocities and forces; leading to complexity in the interpretation of these numbers. Thus it is necessary to explore 
all the underlying mechanisms that govern the behavior of the materials and tap into their properties that are highly desirable for engineering applications and this in turn can be exploited to manufacture "next-generation" materials.

\subsection{Defects in Crystals}

We have discussed how an idealized crystal does not really exist and how all crystals contain defects or imperfections. While the term "imperfection" carries with it the connotation of unfavourable qualities, defects are actually responsible for significant desirable properties in the field of materials science. For example, the usefulness of an ideal silicon crystal in the field of semiconductors would be minimal or close to none without doping it with tiny concentrations of phosphorus and arsenic to give it the desired properties.

There is a fundamental physical reason for imperfection in crystals. Though the perfect crystalline structure is preferred energetically, at the limit of low temperature the atoms are relatively immobile in crystals and hence it is practically impossible to eliminate whatever imperfections are born in the crystal structure during its growth, processing or use. If materials were perfect crystal structures then their properties would be governed by their crystal structure alone, resulting in a limitation in their values and variety.

Crystal defects are divided based on the dimension or geometry of the defect. There are point defects (zero dimensional), line defects (one dimensional) and planar defects (two dimensional). The size of a point defect is close to that of an interatomic space. With line defects, their length is in several orders of magnitude greater than the width, whereas surface defects have a minor depth but their width and length 
may be several orders larger. Volume defects such as pores and cracks may possess substantial dimensions in all measurements.

Processes such as recrystallization during annealing, sintering, oxidation or precipitation hardening may take seconds at elevated temperatures or hours at lower temperatures, whereas phenomenon like creep may take years. These processes are entirely influenced by the behavior of the smallest and fastest moving microstructural defect: the point defect. They are lattice errors at isolated lattice points that occur due to imperfect packing of atoms during crystallization or due to thermal vibration of the atoms at elevated temperatures. They include vacancies and interstitial atoms. Intrinsic point defects such as self-interstitials where in the defect contains only atoms which are the same as those already present in the lattice and vacancies, are present in the crystal lattice at finite temperature due to entropic and energetic reasons. They are formed during the solidification at the solid/liquid interface. It is assumed that they are incorporated at their thermal equilibrium concentration. Upon crystallization, additional mechanisms for the creation are known.

- The Schottky mechanism, where a bulk lattice atom jumps to an interstitial site and diffuses to the surface, where it is added.

- The Frenkel mechanism, where a atom leaves the lattice site by simultaneously creating a vacancy and a self-interstitial.

As the atoms vibrate randomly due to thermal fluctuations, point defects can occasionally migrate from one lattice site or interstice to another. This phenomenon is called solid-state migration. The most probable mechanism of diffusion is one wherein the magnitude of the energy barrier or activation energy [refer Section 3.1] to be overcome by the moving atoms is the lowest. The activation energy depends on the forces of the interatomic bonds and existing defects. For metal atoms, the 
mechanism of vacancy diffusion is the most probable whereas for elements with a small atomic radius such as hydrogen, the interstitial mechanism of diffusion is the most likely pathway.

For most of the diffusion processes, the rate of diffusion can be interpreted in the Arrhenius form whereby:

$$
\text { rate } \propto e^{\frac{-\Delta E}{k_{B} T}}
$$

This implies the rate of diffusion is proportional to the exponent of the activation energy $(\Delta E)$ over temperature ( $k_{B}$ is the Boltzmann's constant). While studying this phenomenon of diffusion, there are two kinds of activation energies that are of interest; the vacancy formation energy which is the energy required to create the vacancy and the vacancy migration energy which is the energy required to move a vacancy between sites. These quantities can be calculated accurately and have been elaborated in chapters 4 and 5 .

The free energy of a larger defect is generally lower than that of a larger number of smaller defects. Therefore the likelihood of vacancies and self-interstitials being consumed by pre-existing agglomerates is more. The study of point defects in metals is based primarily upon the investigation of the physically measurable changes brought out by migration of the defects. Whenever an unstable number of vacancies or interstitials are present in a crystal lattice at a condition wherein they are mobile, they will confront other such defects wandering as the system proceeds towards a state of equilibrium. The strength of this interaction is based upon the kinetics of their migration and the nature of the stable or metastable configuration to which the process eventually leads. Experiments generally require a sufficient density of defects in order to measure the order of physical changes occurring; and thus the analysis of the experiments must be based not only on the migration 
characteristics of the point defect themselves, but also in the way they interact. We have thus studied the interaction of hydrogen-vacancy clusters in nickel by empirical modeling in order to account the behavior of the cluster in a more detailed way.

\subsection{Nickel Metal}

Nickel $(\mathrm{Ni})$ is a transition metal and has found application since ancient times. Artifacts made from metallic meteorites have been discovered dating from as back as 5000 BC like beads in Egyptian Graves [11]. It is the second most abundant element in metallic meteorites after iron. Nickel as an element was not discovered until the 1750 s. In the $16^{\text {th }}$ century, a dark red ore often with a green coating had been a source of problem for copper miners in Saxony, Germany. Their belief was that this was an ore of copper but they were unable to extract any copper from it. In dismay, they named it "kupfernickel" which means "goblins copper" because from the miners superstition there were goblins holding back the extraction of copper. Swedish chemist Axel Cronstedt carried out a number of experiments between 1751 and 1754 to determine the true nature of kupfernickel. When noticing that the results on his work were not in accordance with copper compounds, he isolated a white, hard metal and concluded that he had discovered a new metallic element and named it Nickel after the parent ore. Nickel exhibits a mixture of ferrous and non-ferrous properties. It has good ductility and has a unique ability of reacting with oxygen at an extremely slow rate on the outer layer, leading to the formation of a protective oxide layer and making it corrosion resistant. Nickel is an extremely versatile element and will alloy with most metals. Nickel and copper have complete solid solubility; and a good range of solubility exists with iron, chromium and other common engineering metals. The 
powerful resistance to both heat and corrosion as well as low thermal expansion properties of nickel alloys and Ni-based super alloys have made them useful in domestic as well as industrial applications that require equipment to retain stability and resist corrosion over a wide range of temperatures. Some of the notable applications include aircraft gas turbines [12], steam turbine power plants, medical instruments [13], nuclear power generation equipment and petroleum refineries.

A typical FCC crystal lattice structure of nickel can be viewed in Figure 1.1. If we consider an atom (black color filled spheres) at the center of some face, it has four nearest neighbours that are at the corners of that face, four that are at the centres of adjacent faces in one unit cell and four that are at the centres of adjacent faces in another unit cell; totalling to 12 nearest neighbors.

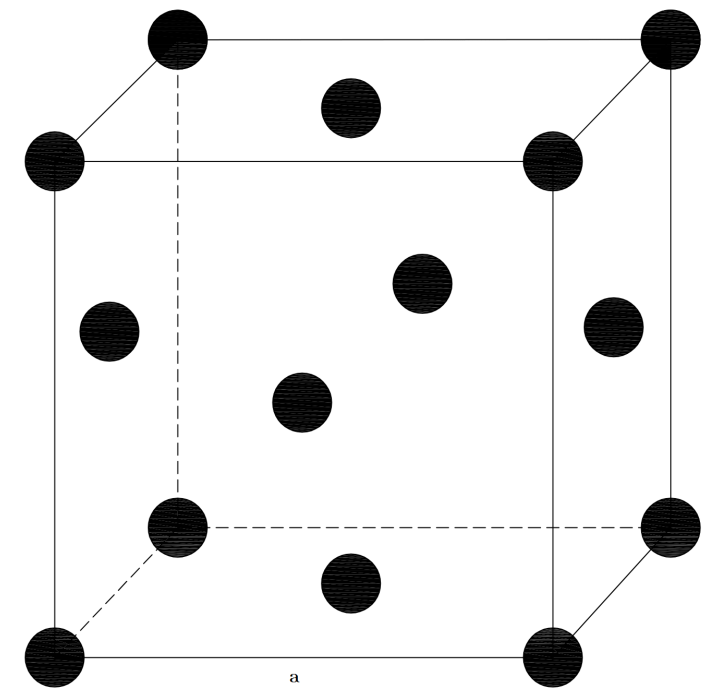

Figure 1.1: Illustration of the FCC lattice arrangement in nickel

The physical dimension of a cubic unit cell is given in terms of the lattice constant (a). It is a parameter that measures the length of the cube edge in the unit cell of a crystal lattice. Here, Ni being a cubic crystal structure has all of lattice constants equal. 


\subsubsection{Interstitial Sites in Close Packed Lattices}

A position that exists between the regular positions in an array of atoms or ions that can be occupied by other atoms or ions is an interstitial site. It is generated as a result of packing of the atoms. There are no atoms completely enclosed by an FCC unit cell; but six face atoms are shared with the adjacent unit cell i.e. $6 \times(1 / 2)=3$ face atoms and eight corner atoms shared like the simple cubic structure leading to one corner atom. Hence an FCC structure contains four total atoms. It is between these atoms the interstitial sites exist.

There are two different types of interstitial sites in an FCC unit cell that depend upon the number of atoms that surround the interstitial site. A group of four atoms form a tetrahedral site as shown in Figure 1.2. The tetrahedral interstitial atoms are shown as non-filled smaller atoms in the figure. During the close packing of atoms, the atom is positioned in such a way that it has contact with three atoms in the layer above it and three other atoms below it, generating a tetrahedral arrangement. The centers of these four atoms lie at the apexes of regular tetrahedron. This results in two tetrahedral interstitial sites per atom. As the size of the atoms increases, the size of the site also increases.

The octahedral site is created by joining six atoms whose centers lie at the apexes of a regular octahedron as shown in Figure 1.3. Looking at the figure, it can be seen that each octahedral site is generated by two sets of equilateral triangles whose apexes point in opposite directions.

Interstitial sites serve as trapping zones for foreign atoms and for lattice atoms that are termed as self-interstitials [14]. An interstitial impurity is one where an atom occupies the interstitial site. A considerable amount of lattice strain results due to this occupation as the atomic plane distorts to accommodate the misplaced atom. 


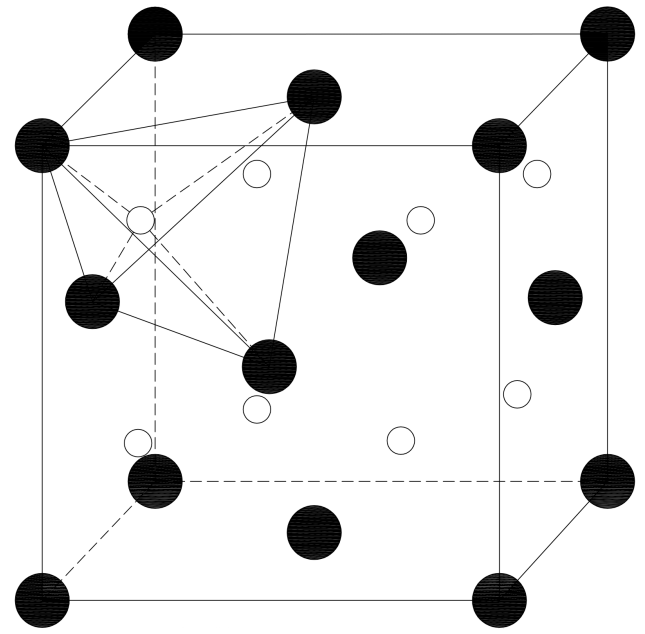

Figure 1.2: Illustration of tetrahedral interstitial sites in an FCC lattice

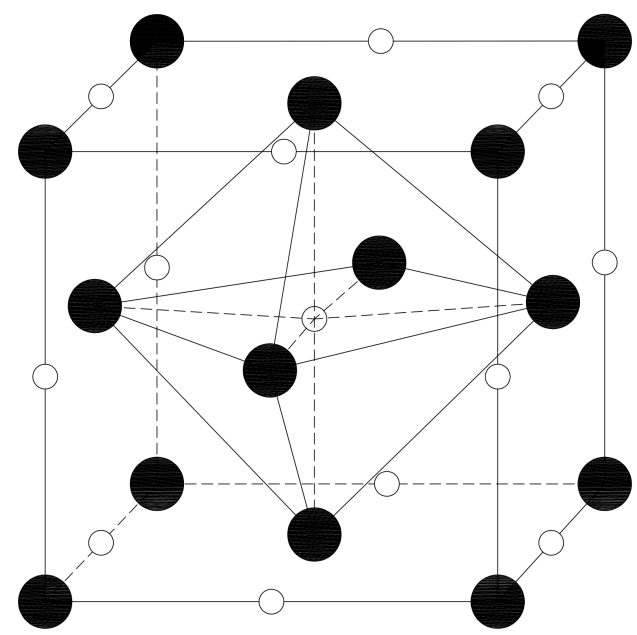

Figure 1.3: Illustration of octahedral interstitial sites in an FCC lattice

The magnitude of the strain depends on the size of the atom in comparison to the lattice atoms.

Concluding, we can say that octahedral sites are those sites that are surrounded at equal distances by six nearest neighbor atoms i.e. a site that lies at the center and the midpoints of the edges in the FCC unit cell. A cell has 12 edges, each of which is shared by 4 unit cells; so that they contribute 3 octahedral interstitial sites per unit cell. In addition, the site that is located at the centre of the unit cell is also an 
octahedral site. Similarly, the tetrahedral site lies at the centre of the space defined by 4 touching atomic spheres. It is formed in the volume that lies between the corner atom and 3 face-centered atoms nearest to it, containing 8 tetrahedral site voids.

\subsection{Hydrogen in Metals}

Hydrogen $(\mathrm{H})$ is by far the most abundant element found on Earth and in its natural state is a di-atomic molecule gas $\mathrm{H}_{2}$. Hydrogen is the lightest element with an atomic structure of one proton and a single electron. It is too large to diffuse in most metals in its molecular state, nor can it willingly cross the gas-metal interface in its molecular form. However not only can molecular hydrogen readily dissolve into molten metal, but it also disassociates once in solution and remains as a mono-atomic solute upon solidification. Due to its lightweight property, hydrogen possesses extreme transport properties compared to helium (both first row occupants of the periodic table) whose smaller molecular cross section compensates for its larger mass [15]. Helium being an inert gas renders hydrogen as the only active 1s element, forcing it to undertake a contradictory function of being both the metal and halogen of the period. This multi role behavior awards hydrogen the broadest range of reactivity of any element, leading to many characteristic types of hydrogen containing systems [16].

\subsubsection{Introduction to Hydrogen Embrittlement}

A known fact since 1866 is that the metal palladium can absorb large amounts of hydrogen gas [17]. The ability of absorbing hydrogen is common to all metals, however the metals differ greatly in how much hydrogen is actually absorbed in a given circumstance. Surface barriers can severely impede hydrogen absorption and desorption 
at room temperature and below which is of significance for many technological applications. Within a metal, the hydrogen molecules are dissociated and the hydrogen atoms occupy interstitial sites in the host-metal lattice. These atoms are capable of migrating from one interstitial site to a neighboring vacant one, thereby diffusing this way over large distances through the metal. A result of the interstitial site occupation is extremely high diffusivity, since the probability of locating a vacant neighboring site for a diffusive jump is more likely.

The introduction and subsequent diffusion of hydrogen in metals leads to a loss of ductility in a material, thereby rendering it susceptible to brittle failure when under high tensile stresses. This process is known as hydrogen embrittlement (HE) or hydrogen induced cracking. The ingress of hydrogen into the metal reduces the load bearing capacity; stress below the yield stress of the susceptible material then causes subsequent cracking leading eventually to brittle failures. The sources of hydrogen include heat treating atmospheres, the working environment, breakdown of organic lubricants etc. The most vulnerable are high-strength steels, titanium alloys and aluminium alloys. Figure 1.4 gives a comprehensive picture on the embrittlement interaction concept. For instance, in the the case of hydrogen embrittlement of stainless steel, hydrogen diffuses along the grain boundaries and combines with carbon, which is alloyed with the iron, to form methane gas. The methane gas is immobile and collects in small voids along the grain boundaries where it builds up enormous pressures that initiate cracks. Hydrogen embrittlement is a primary reason why the nuclear reactor coolant is maintained at a neutral $\mathrm{pH}$ without aluminium components.

At room temperatures, the hydrogen atoms absorbed into the metal lattice diffuse through the grains, tending to cluster at inclusions or other lattice defects which serve as traps. Stress induced cracking under these conditions is transgranular in 


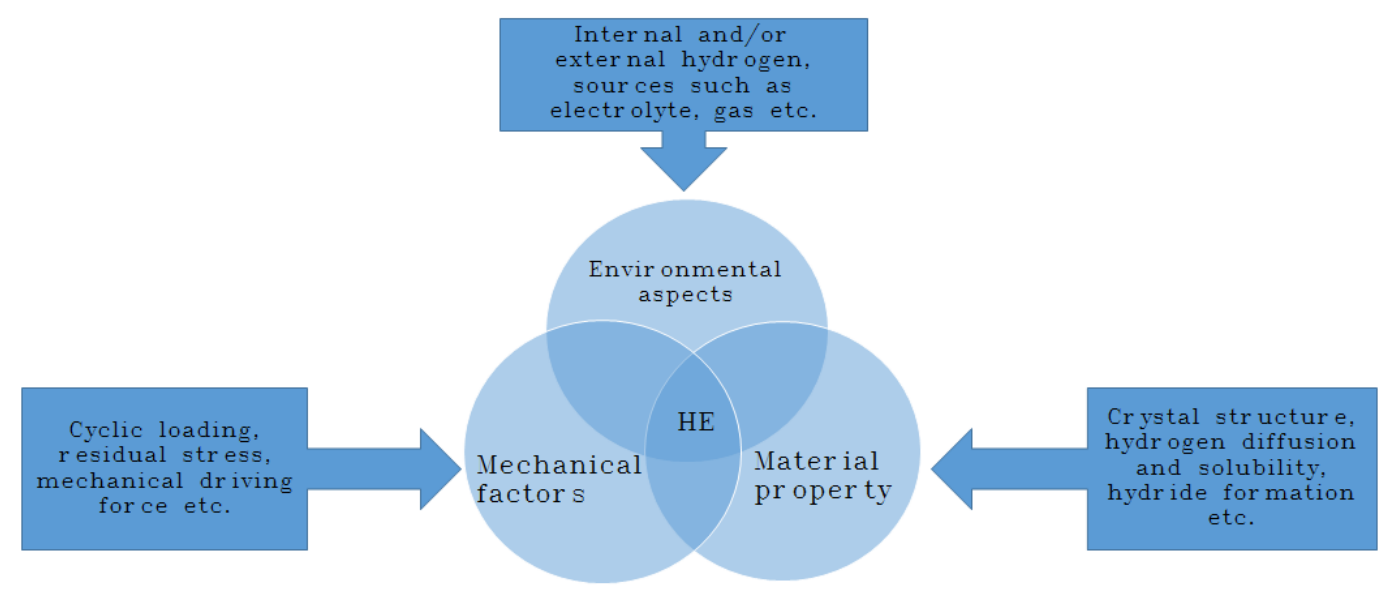

Figure 1.4: Global portrayal of hydrogen embrittlement interaction

path. But at elevated temperatures, the absorbed hydrogen tends to muster in the grain boundaries wherein stress induced cracking is intergranular. The cracking of martensitic and precipitation hardened steel alloys is believed to be a form of hydrogen stress corrosion cracking that results from the entry into the metal of a portion of the atomic hydrogen that is produced in the subsequent corrosion reaction.

Defects in metals serves as traps that handicap the mobility feature of the hydrogen atom. The bigger the size of the traps, the more number of hydrogen atoms that can be accommodated in it; for example a line defect. Further, if two adjacent hydrogen atoms combine to form a hydrogen molecule, the applied stress required to cause movement becomes higher, effectively pinning the dislocation defect to that point. The underlying process to plastic flow is the dislocation movement, implying that there is a reduction in ductility and an increase in the probability of brittle failure by the presence of hydrogen [18]. In addition to reducing the movement of dislocations, solute hydrogen can react with the parent metal to form a hydride.

Hydrogen assisted mechanical degradation of structural materials is a serious problem and has been receiving increasing attention over the last fifty to sixty years. The 
vast number of hydrogen sources (corrosion in aqueous solutions, absorption into pipelines carrying hydrocarbons, contaminants in the melting and welding processes, hydrogen uptake during electroplating or cathodic protection) contributes to the intesity of the problem. The degradation is manifested in diverse ways, such as the catastrophic fracture of ferritic stainless steels to the failure of zircalloy tubing in nuclear reactors [19] by hydride formation.

A number of mechanisms have been proposed to explain hydrogen embrittlement which may vary for a given system, depending on the source of hydrogen and the nature of the applied stress. The following are suggested by Birnbaum [20]: "Nonhydride forming systems such as iron and nickel alloys which do not form hydrides under the conditions in which they are embrittled fail because the hydrogen decreases the atomic bonding (decohesion). In many of these systems the fracture seems to be associated with hydrogen-induced plasticity in the vicinity of the crack tip. Metals such as niobium, zirconium, or titanium, which can form stable hydrides, appear to fracture by a stress-induced hydride formation and cleavage mechanism. Other mechanisms, such as adsorption-decreased surface energy and high-pressure hydrogen gas bubble formation, have also been suggested and may play a role in specific systems" [20].

Hydrogen embrittlement has many facets with wide implications as shown in Figure 1.4. The complicated interactions between the different aspects of hydrogen embrittlement have resulted in a large number of sometimes disputed findings. Most of the research undertaken was focused to solve immediate technical problems whose goal was the development or selection of appropriate materials with acceptable properties for the chosen application and environment. A more realistic approach to be invoked in this study is that hydrogen embrittlement may be governed by multiple 
dominant mechanisms [21]. The complexity and amplification of the problem is governed by structural changes or local damage introduced by the different hydrogenation methods. For example, some trapping sites such as grain boundaries and precipitate interfaces may compete in varying proportions based on the relative coherency of each boundary.

At this point we must admit that much of the hydrogen embrittlement research has been conducted on commercial microstructures which are nearly impossible to fully characterize in the sense of impurity, defect density, etc. As such, experiments have mostly addressed a myriad of competing phenomena without addressing the basic building block i.e. crystal structure and its defects. We propose that the time is right for the crystal structure to be examined in more fundamental ways. In this study we will implement computational models via atomistic modeling [2] that can simulate hydrogen cluster formation around point defects such as vacancies on an atomistic scale. The goal being to generate results that would lead to a better understanding of the hydrogen embrittlement process.

\subsection{Atomistic Modeling and Scheme}

With an aim to investigate the role of point defects such as interstitials and vacancies in serving as hydrogen clustering zones in nickel metal, atomistic modeling is implemented in this study. Conventional theoretical modeling and computer simulations based on the continuum approach such as finite element methods are unable to scrutinize atomic scale defects. Hence continuum models need to be supplemented with atomic models that characterize materials behaviour [22].

Theoretical descriptions of interatomic potential functions [2] are necessary to 
describe atomic interactions, and they play a crucial role in every atomistic simulation. The quality of the simulation results and the amount of time required is governed by the accuracy and complexity of the potential [12]. The density functional theory [DFT] [2] is one of the methods wherein the simulations were directed to find the solution of the complex quantum many-body Schrodinger equation of the atomic system using numerical algorithms. A highly precise description of the quantum mechanical behaviour of the material is supplied by the DFT, but the system sizes that can be studied are limited to only about a few hundred atoms [23]. Moreover the computational resources required to run multiple atomistic simulations is significant, making it impossible to compute the mechanical response of a system of realistic dimensions with this approach. To overcome this hurdle, physicists have developed a more approximate description of the interaction between particles. A simplified form of the atomistic simulation [56] is assumed by semi-empirical models. The ability to obtain quick qualitative results, scan many configurations to obtain trends, sustain longer time scales and to model a larger system in size [24] is permitted by a simple potential. The simplest of such semi-empirical models is the pair potential, where the energy, $E_{\text {tot }}$ of an arbitrary arrangement of $\mathrm{N}$ atoms is written as:

$$
E_{\text {tot }}=E_{0}+\frac{1}{2} \sum_{i}^{N} \sum_{j \neq i} \phi_{i j}\left(r_{i j}\right)
$$

where $E_{0}$ is a constant volumetric energy, $\phi_{i j}$ is an energy potential that depends on the atomic species of both the atoms $\mathrm{i}$ and $\mathrm{j}$ and $r_{i j}$ is the distance between $i^{\text {th }}$ and the $j^{\text {th }}$ atoms. The limitations of the pair potential in terms of its applicability is with regard to the materials it can represent, as well as the range of deformations the material can undergo [25]. A method based on the DFT was derived called the Embedded-Atom Method [EAM] by Daw et al, in order to calculate the ground-state 
properties of realistic metal systems [26]. In the EAM, the energy is calculated as a sum of individual atom energies and the total potential energy $E_{t o t}$, of a configuration of atoms is written as [26]:

$$
E_{t o t}=E_{0}+\frac{1}{2} \sum_{i}^{N} \sum_{j \neq i} \phi_{i j}\left(r_{i j}\right)+\sum_{i} F_{i}\left(\overline{\rho_{i}}\right)
$$

via the equation:

$$
\overline{\rho_{i}}=\sum_{j \neq i} \rho_{j}\left(r_{i j}\right)
$$

where $\rho_{i}$ is the spherically averaged electron density field of an isolated atom. The sums of $j$ are over all atoms within some cutoff radius, $r_{c u t}$ of atom $i$ in both the equations 1.3 and 1.4. This cutoff generally includes the first two or three neighbor distances in a crystal, beyond which it is assumed that the atoms do not interact [25]. The ground state properties such as lattice constant, elastic constant, sublimation energy and vacancy-formation energy $\left(E_{v a c}\right)$ can be calculated from Equation 1.3.

\subsection{Motivation for this research}

Defects can arise in as-grown crystals, during processing (e.g., with iron implantation), or during use (e.g., in the flux of particle radiation in space). Though most of materials are crystalline to a very good extent, atomic arrangements do not follow perfect crystalline patterns. Defects and disorder in condensed matters exert significant influences in the atomic transport properties and mechanical properties, as well as on the crystal's thermodynamic properties. Some basic defects and disorder structures in crystals have been well researched, nevertheless, many uncertainties and areas without bounds remain there concerning the properties of defects in materials. 
This continues to provide some of the most basic challenges in material science and to inspire theoretical and experimental studies.

Defects and disorder are correlated with the fundamental properties of materials, which creates windows for material developers to tailor material properties into the diverse combinations that are demanded by the growing needs of modern engineering. The minuscule scale and complexity of defects in solids often present formidable difficulties for experimental studies. Materials modeling emerges as a new scientific field to bridge analytical theory and experiment to describe and predict the properties of complex systems. The explosion in computer technology over the last few decades has empowered the detailed simulation of complex processes at the atomic level. It is capable of meeting the industry need for solutions that require minimum resources and it is possible to study to single factor via calculations, while experiments always present a mixture of effects.

The vacancy is the most common defect in metals and alloys. The evolution of extended defects and atomic transport phenomenon in materials involve vacancies. These open-volume defects tend to behave as easy traps for impurity interstitial atoms diffusing through the bulk atomic configuration. It is widely accepted that vacancy clusters can develop in materials super saturated with vacancies or even at low vacancy concentration when vacancy-vacancy binding occurs. Hydrogen in the meantime, available abundantly in the atmosphere is an easy target to be trapped either at surface defects or lattice imperfections in solids, thereby playing various roles in altering their properties. Hydrogen embrittlement of metals is a major concern for failure by stress corrosion cracking and fatigue. The conclusive technique of hydrogen embrittlement in metals remains an area of interest and probing for material science researchers since it is not acquiescent to comprehend. Fukai [21] proposes four general mechanisms for embrittlement: i) formation of the hydride phase ii) 
enhanced local plasticity iii) grain-boundary weakening iv) bubble and blister formation. A compelling aspect of hydrogen embrittlement phenomenon is its sensitivity to strain-rate and temperature. Slow strain-rates and moderately elevated temperatures enhance the loss of ductility, which implies that the phenomenon is under the control of and paced by the lattice diffusion of hydrogen. One of the major hurdles faced by hydrogen problem studies involves the inability to quantitatively analyze the critical concentration of damaging hydrogen and it is important to study zones where hydrogen trapping may occur such as voids, vacancies, interstitial sites etc.

Interstitial hydrogen being trapped at a vacancy may lead to the formation of vacancy-hydrogen cluster, which could alter the properties of the point defect from being a interstitial sink to a source; or have an effect in the formation energy of the defect itself. Hydrogen stabilized vacancies can slowly diffuse, cluster and form crack nuclei that can homogenize with main cracks [27], [28], [29]. This mechanism is further supported by the reports by Fukai [21] that multiple hydrogen occupancy of the vacancy can result in extremely high concentrations of the vacancy defect itself. It has been observed in $\mathrm{Ni}$ at high temperature and pressure states [21].

A fundamental evaluation of this mechanism requires a systematic quantification of the hydrogen-vacancy complex/cluster properties, stability as a function of hydrogen concentration, energetics of the interaction, trapping capacity, mobility and diffusion to other crystalline defects.

In this study we try to broaden our horizon of hydrogen-defect interaction, with a hope to understand the probable causes of hydrogen embrittlement. Nickel being a higly ductile FCC crystalline metal, has diverse applications and the presence of hydrogen in its lattice configuration even at low concentrations, can result in significant reduction in fracture ductility accompanied by changes during plastic deformation [30]. The clustering of hydrogen atoms might cause or enhance embrittlement 
which is a point to study. Vacancy migration is a dominant mechanism behind atomic transport, i.e., self-diffusion, in most elemental crystals, and is of fundamental importance in processes like solid phase transformations, nucleation and defect migration. We have specifically tried to explore defect- hydrogen cluster diffusion under loaded conditions in the bulk lattice.

\subsection{Thesis outline}

This thesis is organized as follows. Chapter 2 provides a detailed explanation on the potential energy landscape (PEL), and its relevance whist exploring material configurations, the complexity of the landscape and its features. Specific concepts such as energy minimization and relaxed states, critical to understanding the link between atomistic simulations and the PEL, are given. The role of the PEL while investigating crystal defects will also be elaborated in Chapter 2 .

Chapter 3 provides the background information regarding metal-hydrogen systems. The mechanics of metal-hydrogen and hydrogen-defect interactions along with various studies available in the literature have been brought out here. Theories with regard to hydrogen embrittlement and hydrogen trapping are important aspects to hydrogen-vacancy clusters in this thesis, so their descriptions are in detail. Finally activation energy for vacancy migration is introduced, beginning with the tool implemented to study this phenomenon, the process, and its importance.

In Chapter 4 the detailed scheme and methodology undertaken for this study is exposed. The tools used to perform simulations and the empirical potential implemented to simulate the Ni-H complex have been given in depth here. The preliminary calculations were performed with two goals: prepare configurations for studying hydrogen trapping at the vacancy, and study hydrogen in solution in the perfect bulk, 
since it is the reference state for trapping. The objective is also to reproduce some key quantities from the literature, to firmly establish the agreement between our calculations and those done in the past. The steps involved in investigating the energetics of the hydrogen-vacancy interaction, and the equations implemented in this study, are also covered in detail here.

The research results in this thesis are covered in Chapter 5, subdivided into four catagories: First we bring out the results on the verification of the empirical potential used for this study, second we have reported and analysed the energetics involving the hydrogen-vacancy cluster, third we present certain conclusions on the trapping capacity of a single vacancy based on our findings, and last we extend the energetics from our findings to reporting the influence of applied loads. Last, we discuss additional work and future research directions that will build upon this thesis. 


\section{Chapter 2}

\section{Potential Energy Landscape}

The potential energy landscape is a unifying concept in materials physics and chemistry, which is dependent on interatomic interactions and boundary conditions. Using quantum mechanics or DFT, we are able to calculate the energy of the electrons given the fixed positions of the atomic nuclei, and add to this the Coulomb interactions between the nuclei to get the total potential energy. A potential energy landscape or surface is the relationship - mathematical or graphical, between the energy of a collection of atoms and their arrangement relative to each other in space.

With the development of empirical methods, this electronic energy can be approximated as a potential energy function dependent on only the interatomic distances (instead of the electronic degrees of freedom). The potential energy, $\nu=\nu(\boldsymbol{r})$, of any arbitrary configuration of $N$ atoms, can be computed with the positions $\boldsymbol{r}=\left(\boldsymbol{r}^{1}, \ldots \boldsymbol{r}^{N}\right)$. For a given system (molecule, surface, bulk solid), using a given forcefield, we can calculate the total potential energy as a function of all the coordinates of all the atoms. The set of all possible coordinates $\left\{\boldsymbol{r}^{\alpha}\right\}$ is referred to as the configuration space of our system. Much of this configuration space may be non-physical or close to impractical; but we can simulate virtual atomic configurations on a computer even if they are unlikely to appear in nature. On the other hand, there are noteworthy 
sections of the phase space that tell us a great deal about how materials will behave. For every atomistic model, the potential energy can be written as a sum of terms that depend on the interactions of each atom in the system with its surrounding neighbor atoms.

It is not possible to plot the potential energy function for a system of more than one atom; but it is useful to imagine it as a $3 N$ - dimensional potential energy landscape with topology comparable to the terrestrial landscape around us consisting of mountains, valleys etc. Specifically, much can be learned about the predictions of a potential energy function from the extremal points (maxima, minima and saddle points) of this landscape (refer Figure 2.1).

Considering any point $\boldsymbol{r}_{0}$ in a configuration space, a unique minimum can be associated with it on the potential energy surface by performing a steepest descent minimization (as described in Section 2.3 ). This implies, starting from the initial guess $\boldsymbol{r}=\boldsymbol{r}_{0}$ the following equation is solved (for small values of $\alpha$ until $\nabla_{\mathbf{r}} \nu \simeq 0$ ).

$$
\Delta \boldsymbol{r}=-\alpha \nabla_{\mathbf{r}} \nu
$$

This corresponds to an infinitely rapid quench at zero temperature and the configuration space is divided in this way into disjoint regions, each of which corresponds to the set of all points that quench to the identical minimum. These regions are denoted as basins of attraction and are separated by dividing surfaces and linked by saddle points. This is illustrated in Figure 2.1.

The minima are the darkest regions in Figure 2.1, maxima the lightest regions and the saddle points are denoted as "s". The continuous curves represent constant potential energy and the dashed curves are the dividing surfaces that separate different minima. The dashed curves enclose a region around each minimum which are its basin 


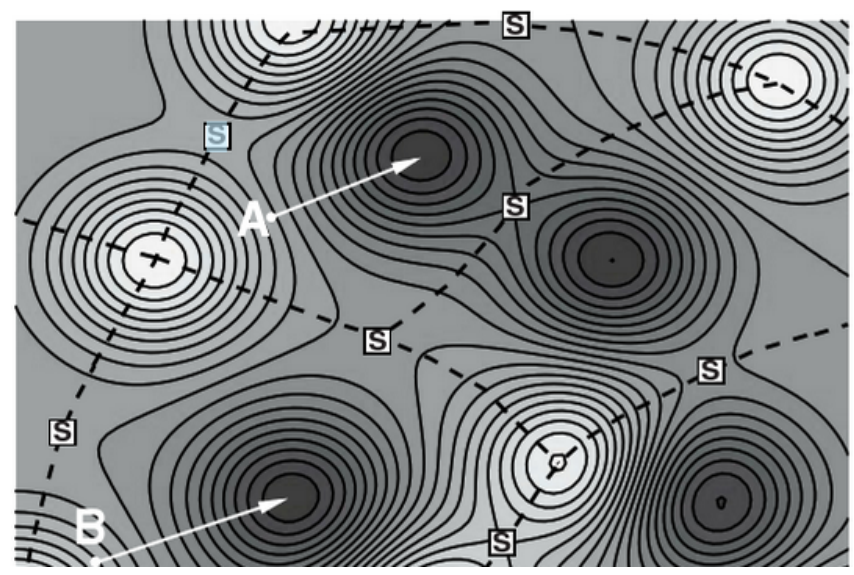

Figure 2.1: Schematic representation of a $3 N$-dimensional energy landscape (Figure reproduced by author from [2])

of attraction. The lowest energy trajectory connecting any two minima is called as the transition path. The transition state at the saddle point along the transition path is unstable (negative curvature).

Defect-free or stable crystalline structures are associated with the deepest minima on the energy landscape. Minima with relatively higher energies are linked with crystals containing internal defects such as interstitials, vacancies, grain boundaries, dislocations etc. Then come an array of amorphous structures and finite clusters of every possible size and arrangement leading to the question of how many minima are actually present on this uneven terrain. A estimate for a system containing only one type of atomic species gives the number of minima as $N ! \exp (\nu \mathrm{N})[31]$, where $\nu$ is some positive number. The first term accounts for particle permutations that yield minima of identical energy and the second term approximates the number of distinct ways of arranging the atomic configurations in stable packings. It is based on the assumption that nearby minima are related through localised rearrangements and hence the total number of such minima is exponential in $N$ [32]. Stillinger et al. [33] approximated that 1 gram of argon would contain $10^{10^{22}}$ distinct structural minima based on this equation. This makes exploring the landscape extremely difficult due to the existence 
of many possible minima and saddle points.

Figure 2.2 shows us the variation in energy when we move an atom in a given system. It can be visualized that the atom has to traverse through varying energy gradients in order to reach a minimum, and the energy landscape comprises of more than one minimum which brings out the significance of the number of iterations that need to be performed in order to arrive at the desired stable minimum.
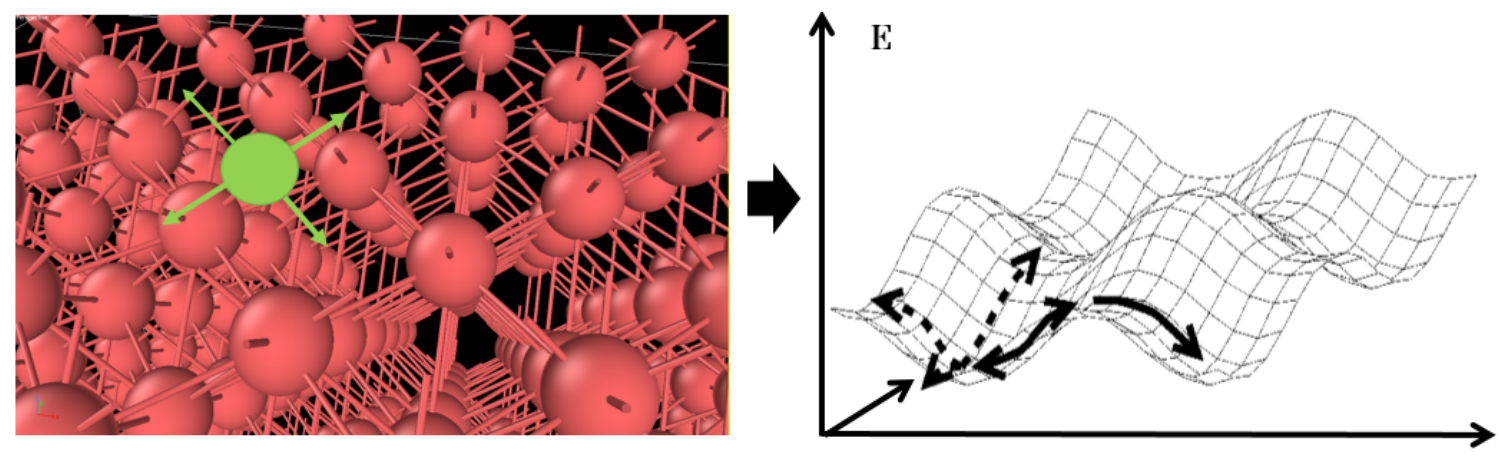

Figure 2.2: Movement of a atom in a $3 N$-dimensional potential energy landscape

The most physically relevant features of $\nu(\boldsymbol{r})$ are the minima and the basins around them. They in turn indicate the energetically stable configurations that the system is likely to adopt and how these atomic arrangements will respond to minor deviations. Given the role of energy minima in understanding materials, methods to search for these minima receive a high degree of importance. A key type of atomistic modeling is molecular statics (MS) (refer Section 2.3) simulation, which is implemented in this study to search for the local minimum energy configurations of an atomic structure. 


\subsection{Features of the Potential Energy Landscape}

For any atomic configuration, we can compute the force acting on each atom from the negative energy derivative with respect to the particle coordinates :

$$
\begin{gathered}
f_{x, i}=-\frac{\partial U\left(r^{\alpha}\right)}{d x_{i}} \\
f_{y, i}=-\frac{\partial U\left(r^{\alpha}\right)}{d y_{i}} \\
f_{z, i}=-\frac{\partial U\left(r^{\alpha}\right)}{d z_{i}}
\end{gathered}
$$

where $r=\left(r^{1}, \ldots r^{N}\right)$ and $i=1,2,3, \ldots . N$.

The above equations can be summarized in shorthand as,

$$
\boldsymbol{f}_{i}^{\alpha}=-\frac{\partial U(\boldsymbol{r})}{d \boldsymbol{r}^{\alpha}}
$$

This expression can be further simplified if the potential energy function is built on pairwise interactions into:

$$
U(\boldsymbol{r})=\sum_{i<j} u\left(r_{i j}\right)
$$

where,

$$
r_{i j}^{2}=\left(x_{i}-x_{j}\right)^{2}+\left(y_{i}-y_{j}\right)^{2}+\left(z_{i}-z_{j}\right)^{2}
$$

The potential energy function $U(\boldsymbol{r})$ defines all of the thermodynamic and kinetic properties of a classical atomic system.

For minima, the gradient surrounding the stationary configuration is positive, i.e. any movement away from this state leads to an increase in the potential energy. For saddles, an atom can move in one or more directions around the stationary configuration and experience a decrease in potential energy. As explained earlier, 
the energy landscape contains numerous stationary points and both the number of minima and saddle points grow exponentially with the number of atoms [32]. The implication of such scaling laws is that it is extraordinarily difficult to search exhaustively for all of the minima for even very small systems (say of the order of few hundred atoms), since their number is so great. By extension, it is extremely challenging to locate the global potential energy minimum, i.e. the set of atomic coordinates that gives the lowest potential energy achievable.

\subsection{Relevance of Locating Minima}

In solids, the global minimum is almost always a perfect crystal. But we are typically interested to examine a local minimum that is nearby some physically motivated starting point for the system. Energetically stable configurations can be identified with the potential energy landscape minima. When studying conformational changes within a atomic configuration, minima give the distinct conformational states possible and the basic structures associated with physical events in the system [34]; which in turn can be compared to experimental analysis.

Defect structures such as dislocations, vacancies and grain boundaries in the bulk crystal possess different minima which are vital in understanding the configurations adopted when a crystal possesses defects. The regions with the lowest free energy are basins around individual local minima, while structures containing defects are also associated with local minima but with higher energies. For example, in the case of vacancies and interstitials, there is a change in the coordination of atoms around the defect when compared to that of a defect-free lattice configuration. This means that the forces are not balanced in the same way as for other atoms in the solid, which 
results in lattice distortion around the defect.

In this study, with the implementation of lattice energy minimization techniques and an appropriate interatomic potential (refer Section 4.1.1), we use energy minimization to explore hydrogen-vacancy complexes, calculate the formation energy of point defects such as vacancy and interstitials; and further investigate the trapping energetics of hydrogen as an interstitial within a monovacancy in nickel (refer Chapter 4).

\subsection{Identifying the Local Energy Minima}

Describing a potential energy landscape in terms of local minima and the transition states that connect them provides a conceptual and computational framework for understanding and predicting observable properties. We have discussed earlier in this chapter that the molecular statics method is one way of arriving at minima on a potential energy landscape. Numerous studies have implemented molecular statics computer simulation methods with an interatomic potential to study defects in crystals. For example the behavior of cracks in $\alpha$-iron [35] has been scrutinised by Shastry et al.; and the energetics associated with self-interstitials and self-interstitial clusters in $\alpha$-iron [36] have been studied by Wirth et al. using molecular statics simulations. The investigation of possible dislocation core structures in nickel-aluminium were studied using molecular statics calculations [37] with an empirical many-body interatomic potential developed within the embedded-atom method (refer Section 4.1.1).

The process of locating the minimum starts with an initial guess close to the expected structure and a mathematical algorithm is run which will hopefully take 
us to a nearby local minimum which is of physical relevance, but we can rarely be absolutely sure. This initial guess is called the "unrelaxed" structure and the process is termed as relaxation where a relaxed atomic structure conforms to an local energy minimum. Rerunning a computational simulation from multiple starting configurations is an ad hoc, but often unavoidable, technique for testing the robustness of a particular simulation. For many problems, there is no clarity on what the initial guess should be and the dependence of the solution on this arbitrary choice is often unnerving. Essentially an atomic configuration is built using a computational software package and energy minimization is undertaken to locate the relaxed structure; also referred to as geometry optimization.

\subsubsection{Boundaries}

If we would like to simulate some properties of a nickel cube say with volume 1 $\mathrm{cm}^{3}$ the number of atoms to include would be approximately $5 \times 10^{22}$. Solving $6 \mathrm{~N}$ differential equations at every time step would be computationally expensive (and likely impossible). The trick to still be able to simulate bulk properties is to use Periodic Boundary Conditions (PBCs). Here we use a simulation box that contains a finite number of atoms. When one atom exits the box at one end it will enter again on the opposite end of the box, as shown in Figure 2.3. That means that the system feels the forces as in a bulk and we can mimic the large infinite bulk with a small system of just a few hundred atoms. Another reason to use PBCs is that the number of surface particles is much larger in a small system and might display unwanted effects. But if we work in three dimensions, having all directions periodic means that we simulate a bulk. If we instead let one of the dimensions being fixed, we simulate a surface. The shape of the simulation box can be of any type if it fills up the entire 


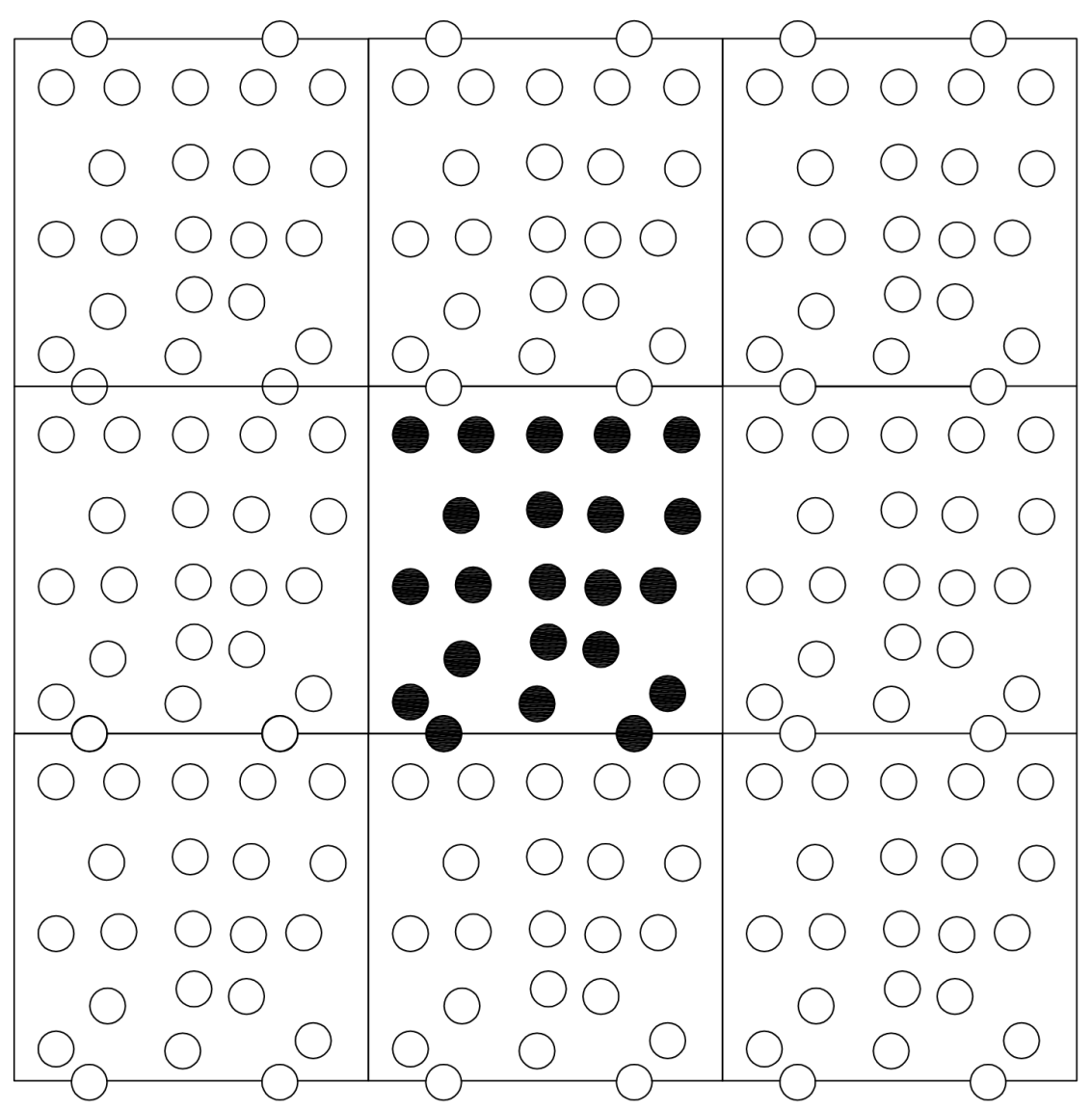

Figure 2.3: Illustration of the periodic boundary condition in two dimensions. When the filled-in atoms exit one end of the simulation box, they enter in the opposite end and in this manner it mimics an infinite bulk structure. The center square is our simulation cell while the rest are images of this.

space using translational operations of the center simulation cell.

In this research, the periodic boundary conditions were used in all simulations, therefore focusing on studying the periodic array of the vacancy defect. A range of simulation sizes were analysed to study the defect interaction effect between the boxes. 


\subsubsection{Steepest Descent Method}

Earlier, we have brought out a brief discussion on the theory and implementation of the most common optimization workhorse in the field of minimization: the steepest descent (SD) method. As the name suggests, the idea is to simply choose the search direction at each iteration to be along the direction of the forces i.e. if the forces are nonzero, we can lower the energy by iteratively moving the system towards a decrease in energy. This corresponds to the steepest "downhill" direction (refer Figure 2.4) at that particular point in the energy landscape.

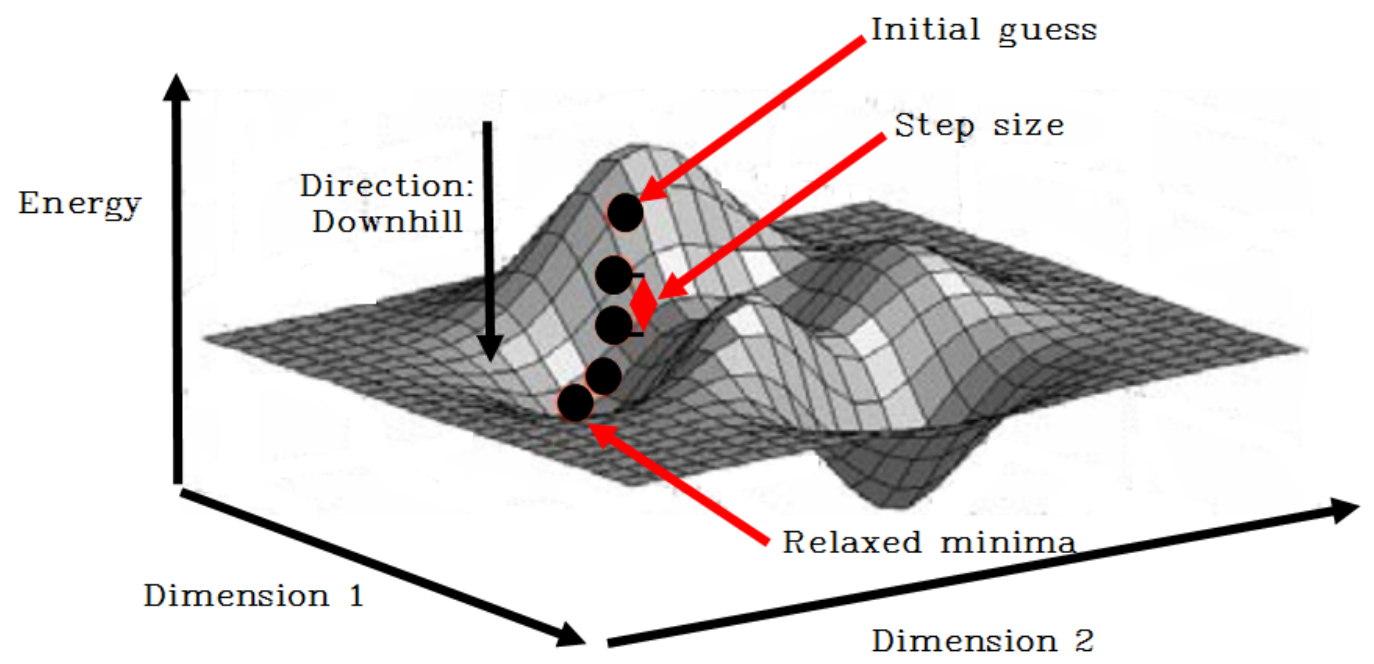

Figure 2.4: Illustration of the steepest descent method implemented on the PEL

The SD method is generally an inefficient and slow approach to finding a local minimum; but it is reliable. The SD trajectory followed in going from the initial state to the minimised state has a clear physical interpretation as an over damped dynamic system, which is significant when one is actually interested in entire pathways in configurational space, as opposed to just the minimum. In the absolutely simplest implementation of the SD method, the step-size can be prescribed to be some fixed, small value, although a check needs to be made to ensure that undertaking the full 
step does not lead to an increase in energy. In more sophisticated implementations, the system is moved some variable amount along the direction of the forces until the one-dimensional minimum along that direction is found. In other words, the multi-dimensional minimization problem is replaced by a series of constrained one dimensional minimizations.

The SD algorithm is an "intuitive" one; meaning that from where you are, move in the local direction of steepest descent i.e. compute a new force, repeat the process until the force norm is below the set tolerance. Thus we can conclude that the method is not especially fast, since for many landscapes the most direct route to the minimum is not in the direction of the steepest descent.

\subsubsection{Selection of Energy Minimization Method}

Locating mechanically stable equilibrium configurations of atomistic systems is one of the prominent tasks in computational material science. To resolve this task, a variety of well-recognised optimization methods exist alongside the steepest descent method; the conjugate gradient, Newton Raphson, quasi-Newton or the truncated-Newton methods [38], [39]. Choosing the right energy minimization style needs evaluation of the computational cost, memory requirements, robustness, ease in implementation (use and parameters) and convergence criterion.

The structural minimization method implemented in this study for arriving at energetically stable atomic configurations (local minima) is FIRE [40], which stands for fast inertial relaxation engine. This technique is very similar to the SD method but with greater control and adaptability in the pathway undertaken to reach the local minimum. It can be explained conceptually by considering a skier scanning for the fastest pathway to the bottom of a valley in an unknown mountain range described by the potential energy landscape. The skier is capable of holding back 
on the downhill progress and steer by introducing acceleration in a direction that is steeper than the current direction of motion if the power is positive, and in order to avoid any uphill climb he simply stops as soon as the power becomes negative. The algorithm arrives at the right direction by letting inertia decide it whilst combining an adaptive time step option to result in a multidimensional minimization scheme.

The FIRE technique has been successfully implemented for the task of atomistic structural relaxation while studying deformation mechanisms in palladium [41], screw dislocations in $\alpha$-iron [42], vacancy-dislocation interaction in tungsten [43] etc. Some of the positive traits of this technique include robust relaxation for all degrees of freedom, low memory usage and computational overhead, gradient-based small convergence criteria and stability [40] with respect to random errors in the potential energy. It scales well with the system size [40]; calculations with up to $38 \times 10^{6}$ atoms have been performed.

\subsection{Application to Crystals and Defects}

The energy landscape is home to higher order local minima associated with defects such as vacancies, free surfaces, grain boundaries and dislocations; which play a central role in the response of real materials, most notably in affecting their strength, ductility and toughness. Molecular statics has aided in understanding some of the fundamental questions about these properties, and these models will continue to help researchers to predict and explain material behaviour in the future. In the coming sections we will address the calculation of a few basic properties of materials using MS. These seed concepts aid in levying an idea of how we utilized atomistic simulation in understanding microscopic details which cannot be readily captured by macroscopic 
observation or experimental work.

\subsubsection{Cohesive Energy of an Infinite Crystal}

Solids often adopt well ordered crystalline structures with well defined lattice constants. It is natural to ask why a given element chooses a particular crystal structure and what are the properties associated with it. When a crystalline solid is formed from infinitely separated atoms, the energy that is released is known as the cohesive energy of the crystal. The interatomic forces that bind the atoms together in a solid are responsible for crystal formation. Hence the energy of the crystal is lower than that of the free atoms by an amount equal to the energy required to pull the atoms to an infinite distance. The typical magnitude of the cohesive energy for crystals varies from 1 to $10 \mathrm{eV}$ per atom.

Mathematically, the cohesive energy of a crystal $E_{c o h}$, is the difference between the energy of a collection of atoms bonded in a crystalline structure and the energy of those same atoms infinitely separated and isolated from each other, divided by the number of atoms in the crystal:

$$
E_{c o h}=-\lim _{N \rightarrow \infty} \frac{\nu_{(N)}^{i n t}-\sum_{\alpha=1}^{N} E_{\text {free }}\left(Z^{\alpha}\right)}{N}
$$

In the above equation, $\nu_{N}^{i n t}$ is the internal energy of the bonded crystal composed of $N$ atoms and $E_{\text {free }}\left(Z^{\alpha}\right)$ is the energy of an isolated atom with atomic number $Z^{\alpha}$. This definition applies equally to simple lattices and multilattices, where $E_{\text {free }}\left(Z^{\alpha}\right)$ allows each interpenetrating lattice to comprise a different species. The negative sign is introduced by convention, so that the final $E_{c o h}$ will be positive for a stable crystal. It is impossible, of course, to model an infinite crystal, but by using PBCs and considering only the energy of the atoms in the simulation cell $\left(E_{\text {cell }}\right)$, the cohesive 
energy becomes simply

$$
E_{\text {coh }}=\frac{-E_{\text {cell }}}{n_{\text {cell }}}
$$

where $n_{\text {cell }}$ is the number of atoms in the simulation cell.

\subsubsection{Crystal Defects - Vacancies}

The starting point for someone who is performing atomistic simulations of crystalline systems is to calculate the cohesive energy of Equation 2.9. The next task would be determining the vacancy formation energy $\left(E_{v a c}\right)$. This is the "cost" for removing one atom from the crystal. It is defined by

$$
E_{\text {vac }}=\lim _{n_{\text {cell }} \rightarrow \infty} E_{\text {cell }}\left(n_{\text {cell }}\right)-\left(-n_{\text {cell }} E_{\text {coh }}\right)
$$

where $E_{\text {cell }}$ is the relaxed energy of the simulation cell containing a single vacancy in an otherwise defect free atomic arrangement and $n_{\text {cell }}$ is the number of atoms present in the simulation cell with a vacancy. In both the simulations the atoms are arranged in perfect lattice positions at the equivalent lattice constants, except for the relaxation effects around the vacancy. The noticeable combination of negative signs is implemented to ensure that $E_{c o h}$ remains a positive quantity while $E_{c e l l}$ is negative.

It can be seen in Figure 2.5 (a), the atomic arrangement is defect free. Hence the $E_{c o h}$ for this simulation cell can be simply calculated by using Equation 2.9, where $E_{c e l l}$ is the relaxed energy of the defect free cell. Figure 2.5 (b) consists of a single point defect vacancy. The energy of formation for a single vacancy is equivalent to the energy required to break the interatomic bonds around one atom. It can be seen how the surrounding atoms reposition themselves around the vacancy during energy minimization. The relaxed energy of the simulation cell containing one vacancy is 


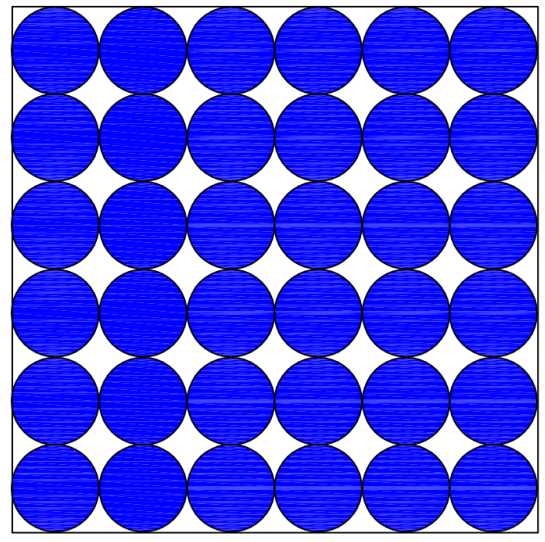

(a)

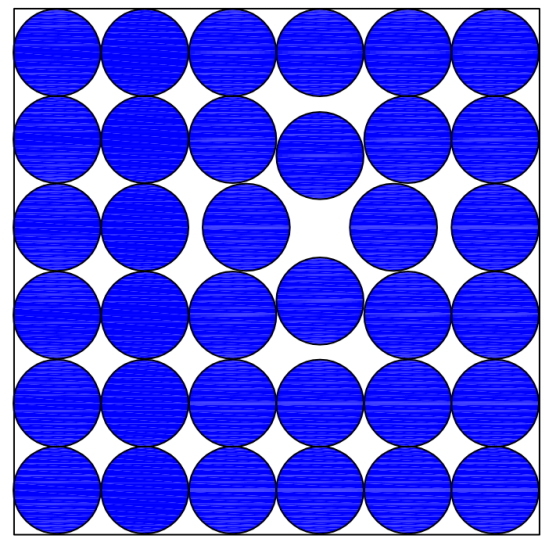

(b)

Figure 2.5: (a)Illustration of a defect free atomic arrangement; (b) Illustration of a atomic arrangement with one vacancy

obtained by energy minimization from which $E_{v a c}$ is calculated using Equation 2.10.

\subsubsection{Crystal Defects - Interstitials}

Interstitials are point defects where in additional atoms (solute or foreign) occupy positions in between regular atomic arrangements at which there is usually not an atom. They are generally higher energy configurations and the interstitial formation energy $E_{i}$ can be calculated in a similar way as $E_{v a c}$ as explained in the previous section.

The energy "cost" for adding one atom into a crystal can be defined as

$$
E_{i}=\lim _{n_{\text {cell }} \rightarrow \infty} E_{\text {cell }}\left(n_{\text {cell }}\right)-\left(-n_{\text {cell }} E_{\text {coh }}\right)
$$

where $E_{\text {cell }}\left(n_{\text {cell }}\right)$ is the energy of a periodic cell with $n_{\text {cell }}$ atoms which contains a interstitial atom in an otherwise defect free atomic arrangement.

The Figure 2.6 (a) is a representation of an interstitial defect where in the same 


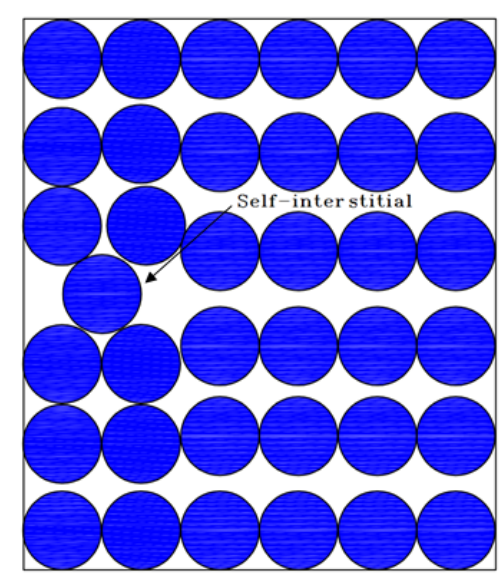

(a)

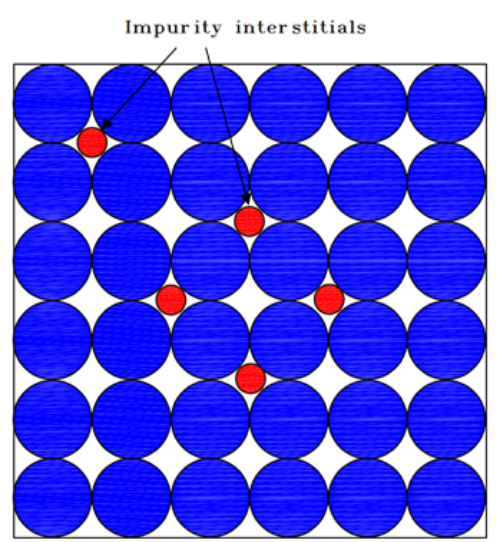

(b)

Figure 2.6: (a)Illustration of the atomic arrangement with a self-interstitial; (b) Illustration of the atomic arrangement with impurity interstitial atoms

type of atom as the others in the bulk crystal arrangement occupies a place outside the normal lattice position (self-interstitial) where as Figure 2.6 (b) is a representation of impurity interstitial atoms (in red color) located within the bulk crystal. The $E_{\text {coh }}$ is calculated as explained in Section 2.4.2 and the relaxed energy of the simulation cell containing the interstitial atom is obtained by energy minimization, from which the $E_{i}$ is calculated through Equation 2.11. 


\section{Chapter 3}

\section{Background Material and Literature Review}

The aim of this work is to analyse the affinity of hydrogen to point defects in bulk nickel and further evaluate the phenomenon of hydrogen-vacancy complexes. The theories of hydrogen embrittlement are introduced briefly, although the focus remains on the studies about hydrogen trapping.

In the year 1866 Thomas Graham, one of the founders of modern chemistry in Great Britain reported that metal palladium $(\mathrm{Pd})$ is capable of absorbing large amounts of hydrogen [17] forming what was known as a metal hydride (MH). A metal hydride is generally defined as a compound formed by hydrogen with a metal. He also observed that the permeation of hydrogen through palladium membranes occurs at an appreciable rate, and thus Pd-membranes can be employed to extract H from a gas stream or to purify hydrogen [44]. Many notable chemists, physicists, metallurgists, etc. have devoted much of their scientific time to metal-hydrogen systems since then. Research on hydrogen in metals has attracted attention for reasons motivated from a basic as well as an applied point of view.

Metal-hydrogen systems have proven to behave as prototypes for certain metal 
properties. Mott and Jones [45] discussed the atomistic processes occurring in the solution of hydrogen in Pd which was the first application of band theory of metals to a chemical process. They are the model for systems in which elastic interaction is responsible for phase transition. The deformation of the lattice due to the trapping of the hydrogen atom in an interstitial site, leading to strain fields that cause macroscopic volume and lattice-parameter changes, is the cause for the attractive interaction leading to the gas-liquid-like condensation [46]. The understanding of the strain field is vital not only for gaining the quantitative insight of the phase transitions of hydrogen in metals but also for issues like hydrogen embrittlement. The strength of interaction with lattice defects such as dislocations, the attraction of hydrogen to a crack tip etc. can be comprehended quantitatively only if the strain field is known [46].

\subsection{Diffusion of Hydrogen in Metals}

It is a well known fact that hydrogen in metals diffuses more rapidly than any other solute. Kehr et al. [47] pointed out that four different diffusion mechanisms should be considered for hydrogen in metals. At higher temperatures hydrogen is localized at specific interstitial positions, subsequently requiring thermal energy to move from that location. The other possibility of hopping involves an activation energy to go over the energy barrier between sites. This is the classical dominant method of diffusion at higher temperatures. At the highest temperatures the hydrogen would populate states above the energy barriers and its diffusion would resemble the diffusion in a dense gas or liquid.

In Figure 3.1, the process path showing how an hydrogen atom (filled in 
Figure 3.1: Illustration of the mechanism of activation energy for diffusion of an interstitial hydrogen atom

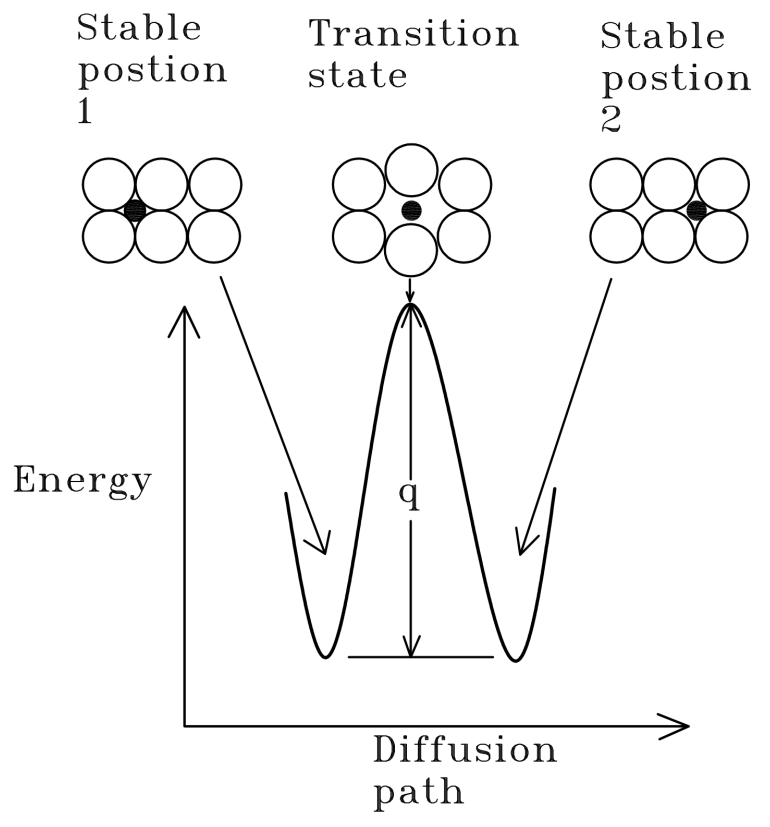

atom) must overcome an activation energy (q), to move from one stable relaxed position 1 to a similar adjacent position 2 is presented. The transition state is the state corresponding to the local energy maximum (most unstable) wherein the hydrogen atom is in an intermediate state of breaking the existing bonds at position 1 and forming new bonds at position 2. In this state, it possesses partial bonds and cannot be isolated as an individual atom. The lifetime of this state is extremely short and it rapidly relaxes to the stable state. The difference between the energy of the starting material and the transition state is called the activation energy. 


\subsection{Hydrogen-Metal interactions}

The enthalpy of solution of hydrogen from the molecular gas into the metal contains two parts: the energy of dissociation of the molecule (independent of the nature of the metal), and the energy of interaction between the dissolved hydrogen atoms, and between the hydrogen and the metal. The latter term varies much from metal to metal and this variation governs the phenomenological separation of metals into the two classes of endothermic and exothermic "occluders" of hydrogen. Measurements of physical properties such as the electrical resistance, the thermoelectric power, electronic specific heat etc. of metals tend to indicate a much larger variation with hydrogen concentration [48] than being simply attributed to the lattice expansion caused by hydrogen. This implies hydrogen upsets the electronic structure of the host metals.

Since hydrogen has an effect upon the electronic structure of the metal in which it resides, as well as increasing the mean separation between the atoms of the host metal, it is not uncommon to find that the cohesive energy between the metal atoms are influenced by the presence of hydrogen. Though no conclusive experiment has been developed in order to measure this effect, a number of theoretical approaches have been reported which demonstrate that hydrogen reduces the cohesive energy in transition metals. A molecular dynamics [2] study conducted on palladium-hydrogen system [49] has reported that the presence of hydrogen reduces the bond strength between Pd atoms by filling in the antibonding states in the $4 \mathrm{~d}$ band. Calculations performed on clusters [50] and the embedded atom method [26] have verified and proved that hydrogen reduces cohesion in many transition metals. 


\subsubsection{Hydrogen-Point Defect Interactions}

The interactions of hydrogen with lattice imperfections are vital and often can be the decisive factor in determining the mechanism of hydrogen embrittlement. Metal lattices exhibit structural defects of various dimensionalities. Vacancies and selfinterstitials can have concentrations consistent with thermodynamic equilibrium, increasing with increasing temperature. Both defects can be generated by plastic deformation and vacancies can be quenched in from high temperatures to produces populations in excess of the equilibrium value. Kirchheim [51] demonstrated that at concentrations of hydrogen in Pd less than $40 \mathrm{ppm}$, instead of the lattice expanding due to the entry of hydrogen, the partial molal volume of hydrogen is a negative quantity. It was also reported in the study that of all the structural defects vacancies provide the strongest attractive interaction with hydrogen in Pd.

The vacancy, being the simplest defect in metals, consists of an empty lattice site with modest peripheral relaxation. Hydrogen is strongly bound to this imperfection in most metals, and the phenomenon has been widely studied in depth using a combination of experimental and theoretical methods [28], [52]. The open-volume character of the defect can be the reason for the existence of an attractive interaction between the interstitial hydrogen and vacancy; in particular when the surface chemisorption state of hydrogen is energetically favored over interstitial solution. In this case the hydrogen is driven to enter the vacancy as the local open volume associated with the vacancy is relatively large, appearing as a free surface to the hydrogen atom. The conclusion being, the binding energy tends to be large and comparable to that for hydrogen in the chemisorbed state [53].

Experiments on metal-hydrogen systems offer clues on the role of vacancies in hydrogen embrittlement. One set of experiments [52] has established that hydrogen

can induce superabundant vacancy $[\mathrm{SAV}]$ formation in various metals. The vacancy 
concentration in these systems can reach a value as high as 23\% [54]. A conclusion drawn from these experiments is that hydrogen atoms, originally interstitial in the bulk, are trapped at vacancies in multiple numbers with rather high binding energies. It has been shown that three to six hydrogen atoms can be trapped by a monovacancy [3], with the highest number of six hydrogens trapped at octahedral sites around a vacancy in either the FCC or the BCC lattice. Evidence also exists showing vacancies interact attractively with hydrogen dissolved in copper [55] and in gold [56].

Lu et al. [3] studied the hydrogen-induced vacancy superabundant formation and vacancy clusterization in aluminium $(\mathrm{Al})$ which lead to the finding that up to 12 hydrogen atoms can be trapped in a monovacancy (refer Figure 3.2); thus leading to a reduction in the formation energy of the point defect itself. This low energy cost to form the defect could lead to an increase in the equilibrium vacancy concentrations thereby creating more trapping sites for hydrogen interstitials. For hydrogen, the tetrahedral site was found to be more favorable than the octahedral site by $0.07 \mathrm{eV}$ in bulk $\mathrm{Al}$ and the hydrogen atom presents affinity towards the vacancy site than the interstitial tetrahedral site by $0.4 \mathrm{eV}$ [3]. They also concluded that the lowest energy position for hydrogen atom inside the vacancy was not the geometric centre but an off-centre tetrahedral site of the vacancy. The zero energy in Figure 3.2 corresponds to the energy of the hydrogen atom at the tetrahedral interstitial site.

It was also summarized in the same study that hydrogen enriched microvoids could be created along the slip planes by the unification of vacancies with trapped hydrogen which could lead to an additional source of microcracks necessary for the hydrogen embrittlement and the lattice mobility of hydrogen atoms is supplemented by the formation of hydrogen-vacancy clusters. The increase in the rate of crack growth results in hydrogen embrittlement due to these vacancy based mechanisms. It has 
Figure 3.2: Trapping energy per hydrogen atom (in $\mathrm{Al}$ ) in $\mathrm{eV}$ as a function of the number of hydrogen atoms trapped in a monovacancy (Image reproduced from [3])

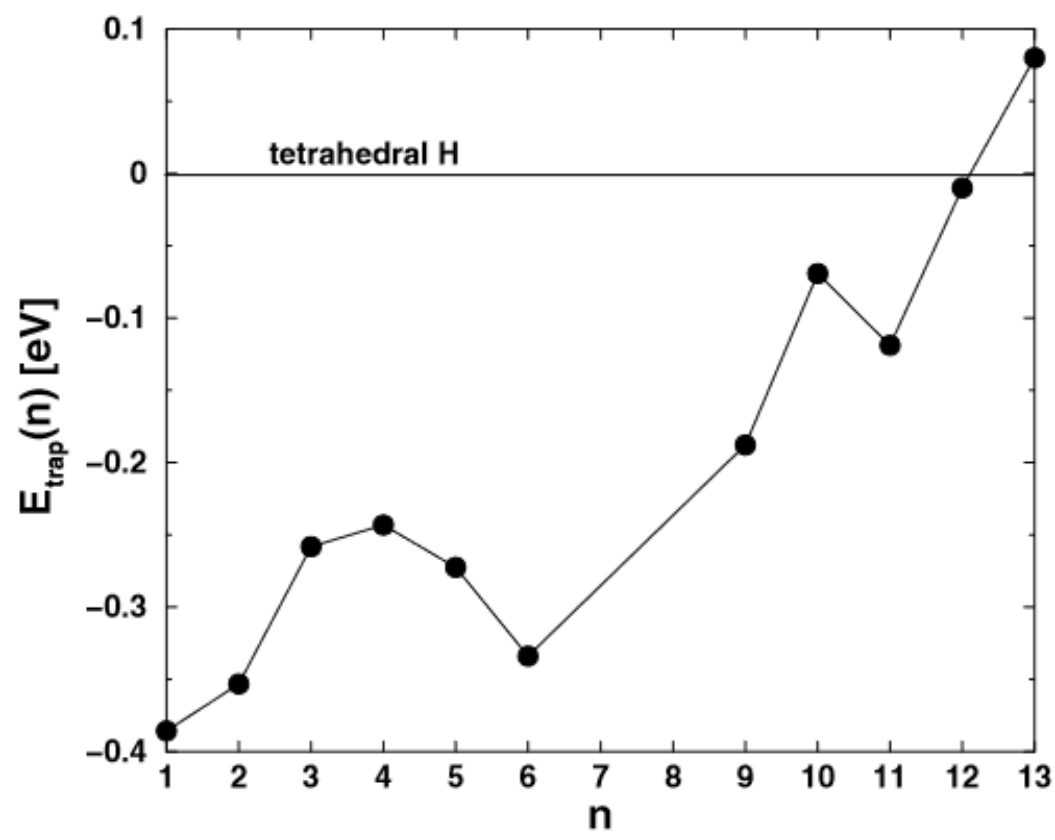

also been reported that hydrogen stabilizes around vacancies [28], [57] and changes the mobility of dislocation defects [58], [59].

In nickel, under high temperature and pressure [60], the formation of a SAV is a result of a reduction in the vacancy formation energy; which implies that defect structures that contain SAVs are in fact the most stable structures of metal-hydrogen alloys. The preferred hydrogen position in the vacancy is the octahedral site, which in turn enables multiple occupancy [61]. The issue of whether these SAVs can result in any kind of environmental degradation of Ni-based alloys is critical; like when at room temperature the non-equilibrium vacancies which are an end-product of plastic deformation and stabilized by hydrogen atoms can have a possible role on the grain boundary cohesion [29]. The hydrogen enhanced strain induced vacancy model is 
in agreement with the primary role of vacancies in hydrogen-related failures rather than hydrogen itself. The hydrogen aids in enhancing the creation of strain-induced vacancies as micro-structure damage during plastic deformation and the SAVs may bond to form further microvoids which can favor crack growth resulting in the fracture process [29]. In this study, nickel was chosen as the material of study because it is a well characterized metal for the purpose of detailed study of individual effects.

Wang et al. [62] performed studies on the trapping of multiple hydrogen atoms in monovacancies, divacancies and at the self-interstitial in Ni. Their main result was that a single vacancy could trap up to six hydrogen atoms in the octahedral positions. The binding energy for tetrahedral sites is always found to be positive, indicating the vacancy is saturated when octahedral sites are completely filled. The analysis of the elastic field induced by the vacancy was carried out which reported that the forces around the vacancy decrease and become negligible beyond $5 \AA$. This implied that the first three shells of the octahedral sites feel the vacancy and, since the force field is oriented away from the vacancy, the interstitial sites are in tension. Hence the resulting stress due to a vacancy attracts small interstitials leading to clustering of hydrogen atoms.

The presence of hydrogen in metal can have drastic effect upon the equilibrium vacancy concentration. When annealing Ni-hydrogen in a hydrogen gas pressure of 5 GPa and high temperatures of $1000 \mathrm{~K}$, Fukai and Okuma [63] discovered that the lattice parameter decreases as a result of high vacancy concentration within the alloys. These SAVs are stable even when the host metal is returned to ambient conditions. The high vacancy concentrations are correlated with a large number of hydrogen atoms trapped near one or more vacancies. Iwamoto and Fukai [64] reported that six hydrogen atoms per vacancy complex in Fe is possible. These additional traps raise the average hydrogen concentration in these metals making them hydrogen storage 
materials.

In our earlier discussion, we have brought out the fact that the defect formation energy is reduced as a result from vacancy-hydrogen bonding and the formation of SAVs. According to Tateyama and Ohno [28], for $\alpha$-Fe there is $30 \%$ reduction in the monovacancy formation energy if two hydrogen atoms are bound to one vacancy. Also the hydrogen-vacancy complex becomes less stable as the number of trapped atoms increases due to repulsive hydrogen-hydrogen interactions. They also investigated the hydrogen-vacancy complex under pressures ranging from $1 \mathrm{GPa}$ to $2 \mathrm{GPa}$ and found out that there was a negative vacancy formation energy, with hydrogen-vacancy complexes containing five and six hydrogen atoms being the most stable.

Recent developments have discovered that vacancies can be simply created by loading the bulk samples with hydrogen. Shirai et al. [65] observed a large increase in the point defect density upon loading a $\mathrm{LaNi}_{5}$ storage alloy with hydrogen. By loading the alloy into the hydride phase, generation of vacancies and dislocations were detected. The trapping of hydrogen, deuterium and tritium by point defect sites like vacancies has been of particular interest in recent years particularly to those studying the penetration of these atoms by ion bombardment. Fusion reactors involve plasma of deuterium and tritium whose energetic ions bombard the walls of the containment vessel and get embedded in the metal surface. Over the course of time, these ions can back out into the plasma, causing problems. The ion bombardment process creates vacancies which act as traps for deuterium and tritium [66].

Point defects, especially interstitials, play a vital role in determining the diverse properties of materials along with the applicability and performance of many technologies [67]. The understanding of site preference and adsorption mechanisms in hydrogen storage materials and defect physics in irradiated materials are crucial to undertake correct evaluation of binding energies. Thus identifying the interstitial sites 
in materials is an important and a long standing problem. Studies have shown that interstitial sites are usually high symmetry positions in a crystal lattice and are determined from symmetry considerations after an extremely thorough visual inspection of the crystal was performed [68], [69].

Geometrical methods have been developed in order to accurately predict the favouritism of the carbon atom to the octahedral site in BCC Fe rather than the tetrahedral site [70] and can serve as a reasonable interstitial starting postion for further relaxations using first-principle methods. Determining the preferential hydrogen-occupying sites in a metal is vital as reported by Tang et al. [71] where the occupancy of interstitial hydrogen in FCC Ni aids in identifying the hydrogendislocation interaction outcomes. Findings report that energetically favourable sites for hydrogen occupation in a perfect Ni lattice are octahedral sites, in the stacking fault are tetrahedral sites and in the Shockley partial core of dislocations [72] are both the octahedral and tetrahedral sites.

\subsubsection{Hydrogen Embrittlement}

The predominant and pervasive effect of hydrogen on mechanical properties is a embrittlement. This implies that with the presence of hydrogen in the metal, lower magnitude of work is needed to cause mechanical failure for a given load-time history. This decrease may be manifested due to the reduction in the tensile strain to result in failure, by the decrease of static load that can be supported by the metal, by a decrease of the number of load-no load cycles, or by an increase of the rate of crack propagation. The most important factor is the ability of hydrogen to reduce the binding forces between the atoms in the bulk which in turn can display itself by a reduction of the force for the normal separation of two portions of a body, leading 
to the easier propagation of a cleavage crack [73], or by decrease in force required for the continuous emission of edge dislocations from the tip of a crack [74] resulting in easy crack propagation by localised plasticity.

Hydrogen embrittlement (HE) is one of the grave issues faced by engineering industries because its susceptibility to hydrogen increases with the strength of steel [75]. The governing mechanism for HE lacks clarity but it is generally accepted that HE involves interaction of hydrogen with defects in the lattice configuration and hence requires the hydrogen to be in a diffusible form [74], [76] [77]. The proposed mechanisms are internal pressure [78], hydride formation [79], hydrogen enhanced decohesion (HEDE) [77], hydrogen enhanced local plasticity (HELP) [74], [80], and hydrogen-enhanced strain-induced vacancy (HESIV) [76] mechanisms.

The presence of hydrogen in crystalline metals has an adverse affect on their mechanical properties. For example, these metals often exhibit a catastrophic failure by limited or no macroscopic ductility before the metal fractures. In high-temperature applications, a hydrogen atmosphere is nearly always present and factors like this contribute to the desire to understand how hydrogen causes failure. The decrease in fracture strength and toughness in many metals due to HE is a well known fact. Thus, understanding the microscopic mechanisms that ultimately lead to macroscopic failures is an important step in attenuating HE. Recent studies have reported that the two micro scale mechanisms regularly linked with hydrogen degradation of Ni are decohesion, where hydrogen at interfaces lowers the cohesive strength, and hydrogenenhanced local plasticity, where it impacts the local instabilities linked with plastic flow [81]. Hydrogen embrittlement of high strength steels and nickel-based alloys is a direct result of decohesion on grain boundaries or interfaces, causing low-toughness intergranular fracture of materials that would normally fail in a ductile manner; embrittlement occurs without notable macroscopic plastic deformation [82], [83]. 
In spite of advancement in the development of materials with remarkable strength and toughness HE remains one of the most severe and controversial types of failure to affect metals whose conclusive mechanism is yet to be understood. The detrimental effect of HE on applications such as the energy sector, water handling applications etc. have led to various experimental studies to understand hydrogen degradation in metals. These range from studies on the effects of hydrogen on slip localization [84], hardening [85], softening [85], hydrogen-dislocation interactions [80] and fatigue [86]. But these experimental results are occasionally conflicting and contradictory. For instance, in $\mathrm{Fe}, \mathrm{Al}$ and $\mathrm{Ni}$, hydrogen has been critiqued to reduce the flow stress [85] while some studies have shown an increase in the flow stress for the same materials [87]. hydrogen has also been linked to the degradation of fatigue properties and fatigue crack growth in steels [86] which is in conflict with more recent results that hydrogen produces fatigue crack growth resistance and can enhance the material's properties [88]. Although such experimental investigations can provide insights on the effect of hydrogen on the macroscopic properties of metals, they are highly dependent upon the experimental setup and unintentional damage that can be introduced into the test specimen, thereby making the findings disputable [84].

Supplementing the previous statements, since HE processes occur on an atomic scale (dislocation core, crack tip etc.), the precise mechanisms leading to HE are not easily identified through experimental procedures. Atomistic simulations possess a unique advantage over experiments in capturing details of deformation and fracture processes at the atomic level, enabling a better understanding of microstructural mechanisms. A molecular dynamics study on the effects of hydrogen on metals was performed in 1986 [89] where it was shown that hydrogen can weaken metal bonds and modify the moduli when present in high concentrations. Similar studies involving atomistic simulations have been undertaken to study dislocation emission 
and plasticity around crack tips [59], [90]. Like vacancies, pre-existing dislocations are ubiquitous in the bulk and play a vital role in controlling the plasticity in metals as the hydrogen-dislocation interaction will affect all the stages of plastic deformation i.e. yielding by onset of dislocation,flow by dislocation motion,hardening by dislocation pinning, dynamic recovery by cross slip, etc. Wen et al. [91] performed molecular statics simulation to study effects of hydrogen on kink-pair formation in $\alpha$-Fe where it was shown that the activation energy for kink-pair nucleation would either increase or decrease based on the transition of hydrogen to various binding sites. Lu et al. [92] reported through ab initio calculations that the stacking fault energies both stable and unstable are reduced by $50 \%$ in aluminium when hydrogen atoms occupy octahedral sites. Also Taketomi et al. [59] through molecular statics simulations showed that the stacking fault energy decreases with the increase in hydrogen concentration in $\alpha$-Fe.

\subsection{Theories for Hydrogen Trapping}

Hydrogen trapping mechanisms and zones can have a crucial effect upon the behaviour of metals. The awareness of trap structures and energies bears on the understanding of gas atom transport, accumulation and bubble nucleation in materials. The defects located in the crystal structure can act either as hydrogen traps or hydrogen repellers [93]. Any material is prone to hydrogen embrittlement based upon the characteristics of its trap population. Reversible traps can behave either as a source or sink depending upon factors such as the initial state of the material (internal or external hydrogen)

and mode of transport of hydrogen. A trap population behaving as a sink is beneficial as it deters the congregation of hydrogen at potential flaw zones [93], but a population 
serving as sources will have damaging effects. Extensive reviews on hydrogen trapping mechanisms and favourable sites can be found in the literature [75], [94].

Both experimental and theoretical studies on multiple trapping of deuterium (D) in a monovacancy [95] in iron has reported that there is direct trapping of D (up to 6 atoms) by defects rather than at defect-strain fields. Investigations [96] carried out using density functional theory (DFT) predict that a single vacancy can store between 6 to 12 hydrogen atoms in tungsten (W) with de-trapping energies dependent upon the fill threshold of the monovacancy. Multiple hydrogen occupancy of the vacancy in FCC structures such as $\mathrm{Ni}$ and $\mathrm{Al}$ has been researched theoritically [97] where in it is reported that up to six hydrogen atoms can be trapped by the monovacancy in $\mathrm{Ni}$, and that the binding energy for the last four hydrogen atoms are significantly lower than the first two atoms since the de-trapping stages come into effect with the reduction in the volume of the open defect. The study also provides a brief upon the existence of a probable hierarchy of traps; the strongest binding energy of the hydrogen is believed to occur at monovacanies [98] and vacancy clusters. The single self-interstitials and interstitial clusters have a weaker interaction with hydrogen but can influence the concentration of hydrogen within the metal by behaving as primary traps once the vacancy defects have a saturated level of filled in hydrogen. The effect of dislocations relatively lacks significance since they are weaker and fewer in number.

The trapping of hydrogen to defects in a nickel lattice such as dislocations and grain boundaries is a widely explored matter. Atomistic analysis was undertaken by Angelo et al. [1] to comprehend the energies which characterize the trapping of hydrogen to lattice defects in nickel. For edge and screw dislocations, the maximum trap site energy was computed close to $0.1 \mathrm{eV}$; for the Lomer-Cottrell areas it is found to be $0.33 \mathrm{eV}$ and at the grain boundaries the binding energy is close to 0.25 $\mathrm{eV}$. The hydrogen segregation is observed to be maximum at the Lomer-Cottrell lock 
and grain boundaries in nickel where as lower extent to edge and screw dislocations.

The interaction of deuterium with two kinds of lattice defects in nickel investigated by Besenbacher et al. [99], [100] reported that vacancies have the highest trap-binding energy of $0.43 \mathrm{eV}$ in comparison with the metal self-interstitials having a weaker energy of magnitude $0.24 \mathrm{eV}$. The calculations also indicate that the $\mathrm{D}$ atom is delocalized when trapped within the vacancy, with a maximum displacement along the direction from the vacancy to the octahedral site i.e. the preferred location within the vacancy being very close to the octahedral interstitial site. The trap-strengths are governed mainly by the interstitial electron density and by any open structures within the lattice that leads to a trap. A study on the interaction of hydrogen isotopes with lattice defects in palladium the by Besenbacher et al. [98] revealed that the vacancies and voids tend to act as the strongest traps and up to six hydrogen atoms can be accommodated in a monovacancy. The study also focussed upon change in trap strengths based upon the occupancy. The most open sites are the ones with the lowest energy, octahedral in FCC. Thus the activation energy for the hydrogen atom to move from one site to another reflecting the minimum density change is the highest in close-packed FCC implying easier diffusion in BCC structures. Any open volume defect will act as a trap for hydrogen since the energy is lowered due to a reduction in the electron density i.e. more open the defect, the higher the trapping energy [98]. The density is less than optimum at the centre of the vacancy and hence the hydrogen is displaced from the centre of the defect towards the interstitial position.

Recently, much research has been carried out using atomistic simulation to reveal aspects of the embrittlement mechanisms [59], [90], [91], [101]. Evidence that the passage of hydrogen is hindered by lattice imperfections which tend to attract and bind it (trapping) has been proven by Smith et al. [102]. Due to its small volume and light weight, it is difficult to study in detail the hydrogen atom in steel and only 
a few studies have reported direct observations of hydrogen (deuterium) [103], [104]. Identifying and studying traps can be useful in confining the hydrogen atoms, thereby preventing them from diffusing to stress concentrations to result in damage.

The possible results of hydrogen trapping in point defects include increased solubility and reduced dimensional changes from hydrogen loading. Zirconium (Zr) vacancies can behave as sinks accommodating up to nine hydrogen atoms [105]. The study undertaken by Wolf et al. [105] reported that the binding energy in the vacancy is more stable when compared to a defect free Zr lattice for up to six hydrogen atoms. But at elevated temperatures, the hydrogen atoms tend to be highly distributed on interstitial sites than trapped in local defects. The study also suggests that self-interstitial defects have the potential to trap hydrogen but have weaker bonding when compared to a vacancy [105].

Along with the vacancies behaving as primary traps, studies have indicated dislocations tend to have some role in interacting with hydrogen leading to possible HELP [78] mechanisms independent of their possible roles in embrittlement. Experimental data [106], [107] supports HELP involving enhanced dislocation motion but the authors of Ref. [106] suggested that hydrogen interacts with other defects in turn modifying their properties to impede dislocation motion. Hence HELP mechanisms were investigated by Song and Curtin [4] using atomistic simulations in Fe, primarily addressing the aspect of dislocation mobility in the presence or absence of hydrogen. They observed that under tension, the hydrogen atoms tend to accumulate at the dislocation defect and form highly concentrated clouds as shown in Figure 3.3. The trapping energy in the dislocation core was found to be close to $0.4 \mathrm{eV}$ which is comparable to the trapping energy range of $0.1-0.6 \mathrm{eV}$ for hydrogen neighboring a vacancy. Under applied shear, they observed that the dislocations pile up against the obstacle and the trapped hydrogen atoms diffuse along with the defect exerting 
a significant resistance to the defect motion.

Figure 3.3: (a)Projected atomistic view of dislocation cores surrounded by clouds of hydrogen atoms; (b) Close proximity atomistic view of a dislocation core surrounded by hydrogen atoms. Figure reproduced from [4]

(a)

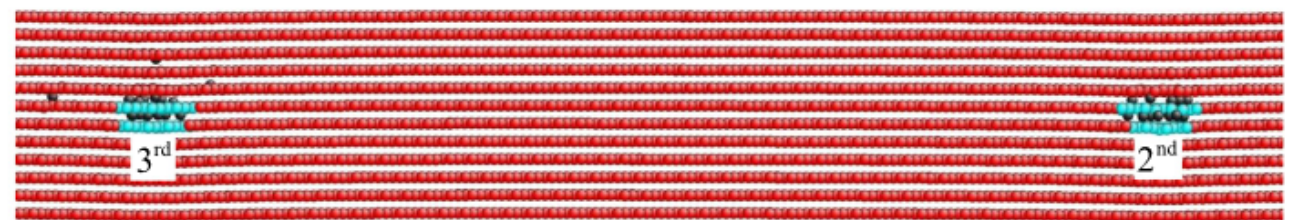

(b)

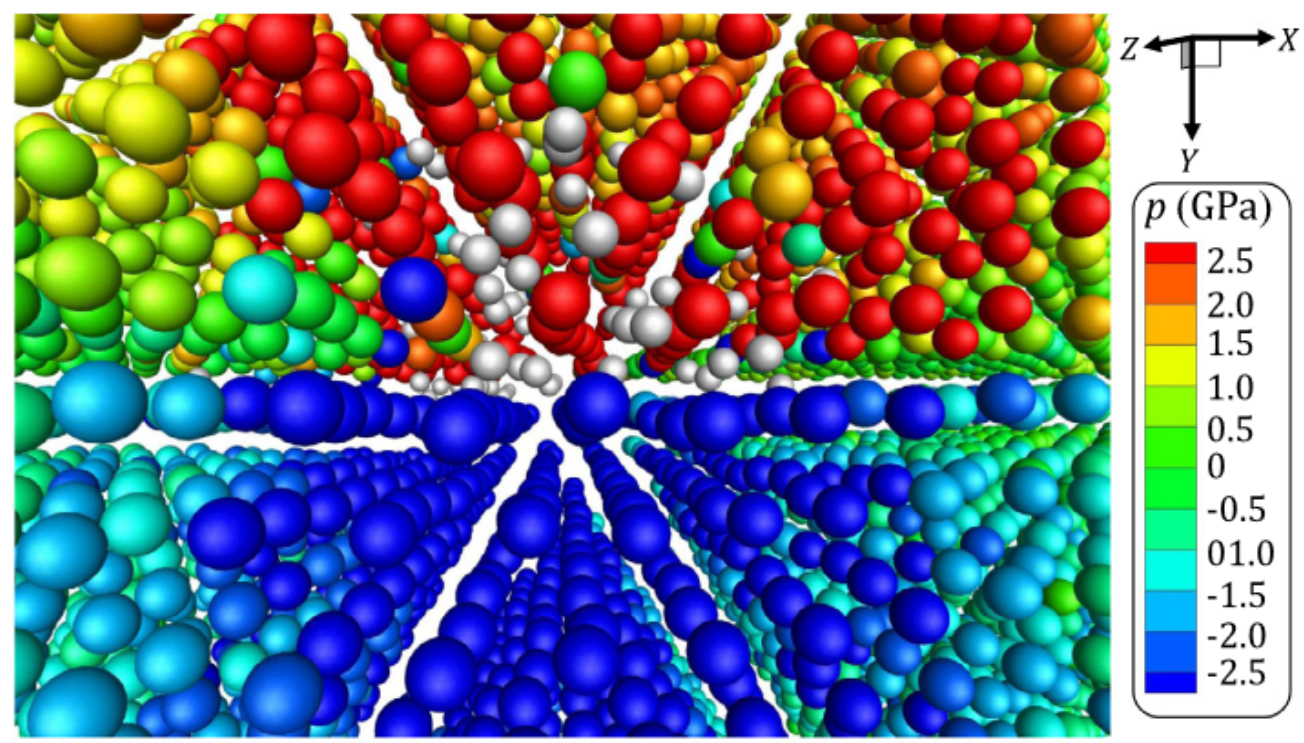

The hydrogen atoms in Figure 3.3 are represented by silver color and the Fe atoms are colored with respect to the local atomic level stress, with positive and negative values indicating tension and compression. The low dislocation velocity due to solute drag suggested its dependence on hydrogen kinetics. Their findings were fairly consistent with solute drag [108], wherein the velocity is controlled by a balance between the applied force and the retarding force of the diffusible hydrogen cloud along with the dislocation. Though the activation energy for hydrogen diffusion to occur in Fe is very low $(\sim 0.04 \mathrm{eV})$, molecular dynamics [2] was implemented to observe the phenomenon. Their other findings included the direct hydrogen effect on the 
dislocation mobility and the no apparent shielding effect of hydrogen on dislocation - dislocation interactions. The findings of this group motivated us to broaden our spectrum of investigation from hydrogen trapping at a vacancy in nickel [2] to the mobility analysis of the hydrogen-vacancy cluster under pressure.

Experimental studies [29], [57] have shown that hydrogen enhances the formation of strain-induced vacancies in body-centered cubic and face-centered cubic metals and alloys. Atomistic simulations of nanoindentation [109] in nickel have reported that vacancies can significantly facilitate the onset of plasticity. Considering that vacancies are the most evident and common defects occurring in crystals, their presence and interaction with other defects can be of interest in the presence of hydrogen.

The findings of various studies made us ponder upon the idea of identifying the optimised hydrogen trapping positions within the vacancy and to analyse accommodation capacity of a monovacancy. Since previous investigations [99] [100] revealed vacancy-interstitial sites are favourable in nickel, further questions originated like what could happen if we continue filling in the all the interstitial sites (octahedral and tetrahedral) within the vacancy, whether the first nearest neighbour interstitial sites are more likely to be favoured, would the vacancy binding energy be lower than the first nearest neighbor sites, does system size have an effect, etc. The research was carried out without the effect of temperature and molecular statics [2] was implemented to obtain the relaxed state of the atomic configuration with the point defects. Since DFT is too computationally expensive to handle large scale systems [23], we investigated the trapping effect with respect to system size using an empirical embedded-atom method potential. The detailed methodology undertaken is explained in Chapter 4 of this thesis. 


\subsection{Activation Energy for Vacancy Migration}

Several micro-structural changes in solids takes place through solid state diffusion, i.e. the movement of atoms in solid phases. Materials science phenomena such as defect diffusion involve stochastic processes associated with the diffusion of atoms over barriers that are high with respect to temperature i.e. the process is slow when the barrier is high compared to temperature $\left(\left(E / k_{b} T\right) \gg 1\right.$, refer Equation 1.1) and hence may occur on time scales that are well beyond the reach of standard molecular-dynamics simulations. At low temperatures, micro-structural dynamic activity in materials occur by activated processes (a sequence of jumps), in which the energy barriers crossed are high. As explained in Chapter 2, on the PEL, the force is negative of the gradient of the potential energy which makes the atomic configuration continuously move towards a nearby local minimum (point from which a minor change increases the energy in all directions on the phase space).

The Figure 3.1 (refer Section 3.1) is suitable in the circumstances of low temperatures, where activated processes dominate relaxation and diffusion. In this state, the lowest-energy paths have barriers many times higher than the average thermal energy and jumps from one local minimum to another are sparse. Establishing the three key points along the path; the minimum, the saddle point and the new minimum provides information about the role of defects, the diffusion mechanism, etc.

By definition, the exploration of the potential energy landscape constitutes a change in configuration which is quite complex especially since the atoms have access to a continuous space. Different methods exist exits to identify transition points and a method which concentrates on vital changes that take place in the microstructure especially under transition state and search for saddle points whilst exploring the 
landscape is the activation-relaxation technique (ART) [110], [111], [112]. The technique is self-evolving in nature wherein it follows the well-defined paths (transition pathways) between local energy minimum.

Starting from a relaxed local energy minimum, the approach treats the minimum energy path as a path following the bottom of a valley to an adjacent saddle point. This comparison is realistic since any displacement away from the minimum energy path increases the energy. The algorithm displaces the first nearest neighbour shell of atoms. The force is the first derivative of the energy and the curvature of the PEL is the second derivative of the energy as a function of the coordinates. ART works by displacing the atoms until a negative second order derivative i.e. negative curvature is detected. It then moves the configuration in the direction of the negative curvature and away from the original minimum while minimizing the forces in the normal direction to the displacement. This implies that the configuration is pushed away from the initial minimum and then relaxed. This action ensures that we do not fall back into the original minimum by accident. In this study we have employed the kinetic activation-relaxation technique ( $k$-ART) [113], [114], [115] which applies the ART algorithm to find the activated mechanisms.

The ART technique has been applied to study self-interstitial clusters [116] ranging from monointerstitial to quadri-interstitial in iron where interstitial self-diffusions have been examined to determine low energy configurations. Investigations carried out on the neutral vacancy diffusion in bulk silicon [117] at zero pressure using ART reported the activation energy for the diffusion mechanism in Si to be $0.32 \mathrm{eV}$, which is in good agreement with previous studies [118], [119]. The $k$-ART package has proven its reliabilty in studying point defects and vacancy diffusion pathways in $\mathrm{Si}$ [120]. The diffusion mechanisms and pathways for one to four carbon interstitials in Fe and a monovacancy coupled with several carbon interstitials has been researched [121] 
using the $k$-ART package.

In this study we utilize ART in order to examine the diffusion energetics of the vacancy and hydrogen-vacancy cluster in nickel. We investigate the effect of the hydrogen cloud upon the vacancy defect during diffusion and try to understand if there is any effect on the diffusion process when the system is loaded or stressed. Essentially we aim to simulate a situation where the interstitial hydrogen atoms if clouded around the vacancy have any tendency to migrate towards a stressed zone in $\mathrm{Ni}$ as per reported findings in $\mathrm{Al}$ [4]. The results and analysis are presented in Chapter 5 of this thesis. 


\section{Chapter 4}

\section{Methodology}

Understanding the hydrogen-defect interaction in crystals is important for evaluating whether it carries the potential to affect the embrittlement process. The prior chapters provide the necessary background information with regard to the importance of studying crystal defects, the role of atomistic modeling in understanding microscopic effects (which in turn have influence on macroscopic properties), the effect of hydrogen in metals, and theories relevant to hydrogen trapping by point defects. The purpose of this study is to research the affinity of interstitial hydrogen to the vacancy defect in bulk nickel, and to evaluate the possibility of hydrogen-vacancy cluster formation. We then study the diffusion of the cluster under applied loads. The study builds upon the findings of previous studies which have been covered in chapters 2 and 3 . The exploration of the potential energy landscape, implementing a suitable energy minimization technique, choosing a robust interatomic potential and applying these concepts first on a pilot study to then broaden the horizon of our study forms the backbone of the methodology adopted.

This chapter includes all the techniques, calculations and software details. How to reproduce the simulations performed in this thesis, should be clear after reviewing this part. 


\subsection{Simulation Setup and Design}

The central tool in this thesis is the Large-scale Atomic/Molecular Massively Parallel Simulator (LAMMPS) software [122] which is a freely distributed classical molecular dynamics code developed by Sandia National Laboratories. The parallel Message Passing Interface (MPI) and the Serial executables were downloaded from the LAMMPS webpage [123]. The simulations have been done in a parallel environment on one central cluster, the High Performance Computing Virtual Laboratory (HPCVL) located at Queen's University. Post processing of LAMMPS output data is handled by Python and MATLAB scripts we wrote to compute the physical properties of interest. Additionally, the Open Visualization Tool-Ovito [124] provided a means to visualize the LAMMPS output data and debug any problems in LAMMPS input scripts. In our simulations, multicore processors were used to take advantage of the parallel processing capabilities of LAMMPS.

The properties predicted by an atomistic simulation are only as reliable as the quality of the underlying interatomic potential. The embedded atom method (refer Section 1.5) is a recently developed form of interatomic potential that has lead to considerable improvement in the quality of prediction for metallic and intermetallic properties.

\subsubsection{Interatomic Potential}

In classical simulations, the atoms are represented by point-like centers, which interact though many-body interactions defined by the interatomic potential. In this manner, the highly complex description of electron dynamics is replaced by an effective model whose main features such as the hard core of the atoms and internal degrees of freedom are described by a set of parameters and analytical functions, which depend on the 
mutual positions of the atoms in the configuration. These parameters and functions give complete information about the system energy, as well as about the forces acting on each particle.

The best choice of a potential for simulations of metals is a many-body potential, since pair-wise potentials such as the Lennard-Jones (LJ) [125] potentials do not provide sufficient description of all properties of metals. For example, the LJ potential imposes the Cauchy relation $\frac{C_{12}}{C_{44}}=1$ on the elastic constants; but the ratio of the elastic constants is far from unity for most cubic crystals. Metallic bonding, like covalent bonds, arises from the sharing of electrons, which implies that an accurate description is required where many-body effects are to be considered. Pair-wise potentials fail to estimate the structural relaxation and reconstruction around point defects in metals. A good example being the vacancy formation energy obtained by means of pair-wise potentials is overestimated, and is found to be almost equal to the bulk cohesive energy.

Daw et al. [26] reported that by adding the embedded energy term for every atom to the pairwise potential an improvement in simulation results is observed over pair potentials. Using a similar approach, Angelo et al. [1] fit an interatomic potential adopting the embedded atom method (EAM) to study the interaction of hydrogen with various lattice defects in nickel. This potential can treat FCC Ni, FCC Al and $\mathrm{H}$ [1]. Details of the fitting procedure are presented in the corresponding embedded atom method paper published by Angelo et al. [1]. The potential gives an accurate fit to the experimental values of lattice constant $\left(a_{o}\right)$, cohesive energy $\left(E_{c o h}\right)$ and bulk modulus $(B)$ for any choice of pair potential $(\Phi(r))$ and electron density $(\rho(r))$. Other material properties such as elastic constants $\left(C_{11}, C_{12}, C_{44}\right)$, vacancy formation energy $\left(E_{v a c}\right)$, the bond length and migration energy show good agreement with experimental results [1]. This EAM potential is considered to be an accurate potential available 
in literature for nickel-hydrogen $(\mathrm{Ni}-\mathrm{H})$ systems [90] [126]. It has been successfully employed in molecular statics using LAMMPS for the study of hydrogen interaction with dislocations in nickel metal [71].

Table 4.1: Comparison of the calculated values of nickel from the EAM potential with the experimental values (Table reproduced from [1])

\begin{tabular}{|c|c|c|}
\hline Parameter & Calc. & Experimental \\
\hline$a_{o} \AA$ & 3.52 & 3.52 \\
\hline$E_{\text {coh }} \mathrm{cm}^{-2}$ & 4.45 & 4.45 \\
\hline$C_{11} 10^{12} \mathrm{dyn} \mathrm{cm}^{-2}$ & 2.464 & 2.465 \\
\hline$C_{12} 10^{12} \mathrm{dyn} \mathrm{cm}^{-2}$ & 1.473 & 1.473 \\
\hline$C_{44} 10^{12} \mathrm{dyn} \mathrm{cm}^{-2}$ & 1.248 & 1.247 \\
\hline$E_{\text {vac }} \mathrm{eV}$ & 1.59 & 1.6 \\
\hline$E_{\text {mig }} \mathrm{eV}$ & 0.46 & 0.41 \\
\hline
\end{tabular}

In this work, the $\mathrm{Ni}-\mathrm{H}$ interactions were modeled using the EAM potential (Ni-AlH) developed by Angelo et al [1]. The potential was downloaded from the OpenKIM Knowledge for Interatomic Potentials [127] website.

Table 4.1 adopted from [1] shows the experimental data used in the fitting parameter along with the calculated values from the EAM potential for nickel.

\subsubsection{Simulation Technique and Methology}

In this study, we employ a molecular statics (MS) technique for atomistic simulation. Using the MS technique with the EAM potential, it is possible to address questions about the relative stabilities and structures of $\mathrm{Ni}-\mathrm{H}$ interactions. As explained in Chapter 2, MS refers to the mechanism of finding the atomic geometry that corresponds to the lowest energy for a given defect specification. These energies and structures thus correspond to the classical zero-temperature system, representing a 
good approximation at low temperatures (one-third melting temperature).

This section is organized as follows: In the first section, the method is exposed. Then, preliminary calculations are performed with two goals: prepare configurations for hydrogen trapping at a monovacancy, and study hydrogen in solution in the perfect bulk, since it is the reference state for trapping. In this stage we research several sites that are in competition for trapping the hydrogen. The objective is to reproduce some key quantities that appeared in literature to firmly establish the agreement between our molecular statics calculations and those done in the previous studies. Some of the preliminary findings have been reported in this chapter which were necessary for progression of this thesis study methodology. Later comprehensive calculations of $m$ hydrogen atoms in a vacancy are presented where we try to investigate the hydrogenvacancy interaction and cluster formation. The goal is to establish the maximum trapping capacity of the vacancy and to extract the typical trapping energy value that can be compared to other studies. In the last stage we research the effect of stress upon the hydrogen-vacancy cluster.

Initially, properties of defect free bulk $\mathrm{Ni}$ are calculated and compared with those in literature. In order to verify that the $\mathrm{Ni}-\mathrm{Al}-\mathrm{H}$ potential accurately describes the $\mathrm{Ni}-\mathrm{Ni}$ interactions, the cohesion energy $\left(E_{c o h}\right)$ and lattice parameter (a) were calculated for bulk $\mathrm{Ni}$ by implementing the EAM potential in LAMMPS. To accomplish this, a simulation cell containing a FCC Ni lattice was defined. The size of the cell spanned 5 lattice units in each direction $(5 \times 5 \times 5)$ and contained $500 \mathrm{Ni}$ atoms. All the ground state properties of FCC nickel and preliminary calculations in this study were undertaken on this supercell first. The bulk simulation is periodic in all three directions at 0 Kelvin. The lattice parameter is expressed in terms of atomic volume. The simple cubic super cell has the volume $V=a^{3}$ and this volume divided by the number of atoms gives the atomic volume. The nickel FCC cell contains four atoms 
in each supercell (refer Section 1.3.1).

From the literature we were aware that the lattice constant fit into the potential was $3.52 \AA$. By varying the lattice parameter and calculating the energy of the system using LAMMPS, an energy-volume curve is generated. This curve is fit to an analytic form (Birch-Murnaghan Equation of State [128]) from which the equilibrium lattice parameter and equilibrium cohesive energy can be directly estimated. Both the values are vital for calculating the formation energy of defects in bulk $\mathrm{Ni}$ and in estimating the trapping energy of hydrogen at a monovacancy. Their relevance in implementation will be brought in the further sections of this chapter. The results from this stage of study has been exposed in Section 5.1 of Chapter 5 in this thesis.

\subsubsection{Preliminary Calculations}

In this section, we present the main properties of vacancies and hydrogen in solution.

\section{Single vacancy}

First, a single vacancy is studied. Using the equilibrium lattice parameters calculated from the analysis of the defect free $\mathrm{Ni}$, a single atom is removed from the supercell and energy minimization is performed. Figure 4.1 schematically shows the open-volume (space within the dotted circle) created in the absence of a regular $\mathrm{Ni}$ atom in the bulk lattice. The formation enthalpy of an $n$-vacancy $\left(H_{n v}^{f}\right.$, where $n=1,2$, etc.) is calculated. Since the supercell is relaxed (the pressure on the supercell is equal to zero), the formation enthalpy is equal to the formation energy of the vacancy $\left(E_{n v}^{f}\right)$.

The $E_{n v}^{f}$ was calculated as follows:

$$
E_{n v}^{f}=E_{0}[N \cdot N i+n V]-E_{0}[N \cdot N i]
$$




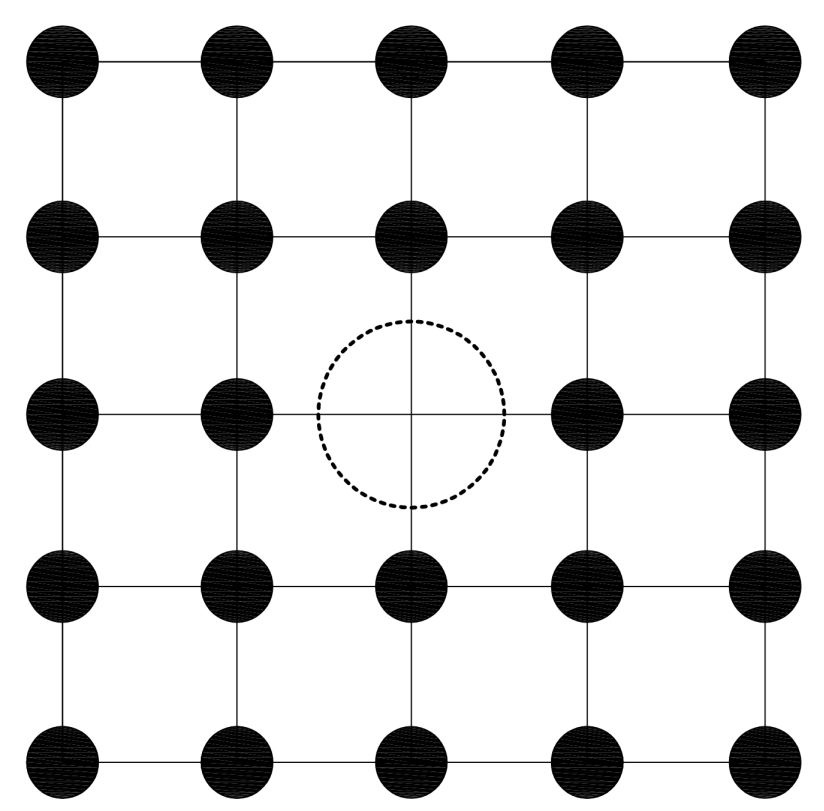

Figure 4.1: Illustration of the formation of a single vacancy in a perfect lattice configuration

where $E_{0}[N \cdot N i+n V]$ and $E_{0}[N \cdot N i]$ correspond to the energy of the relaxed super cell of a system with $n$-vacancies and that of the relaxed supercell of a system without any vacancies (i.e. $N$ nickel atoms).

\section{Hydrogen in solution}

Our main objective is to understand the atomistic mechanisms of hydrogen-vacancy interaction in $\mathrm{Ni}$. In order to compute the solubility of $m$ hydrogen atoms in $\mathrm{Ni}$, we need to study the difference between two states; first, where the hydrogen atom is inserted in the bulk lattice and the second where the hydrogen atom is under the influence of the vacancy. In both the states we address the relative site preference of the hydrogen atom towards the interstitial sites in competition i.e. the octahedral 
and the tetrahedral. The equation for the insertion $\left(E_{i}[H]\right)$ energies according to the site being evaluated is given by:

$$
E_{i}[H]=E_{o}[N \cdot N i+m H]-E_{o}[N \cdot N i]
$$

where $E_{o}[N \cdot N i+m H]$ corresponds to the energy of the system with $m$ interstitial hydrogen atoms in the lattice.

The Figure 4.2 (a) shows the schematic insertion of the hydrogen atom $(H)$ as an interstitial into a 2-dimensional perfect atomic configuration. The Figure 4.2 (b) is an illustration of the interstitial hydrogen (smaller size atom) at the octahedral $(O)$ and the tetrahedral $(T)$ sites of the FCC Ni lattice.
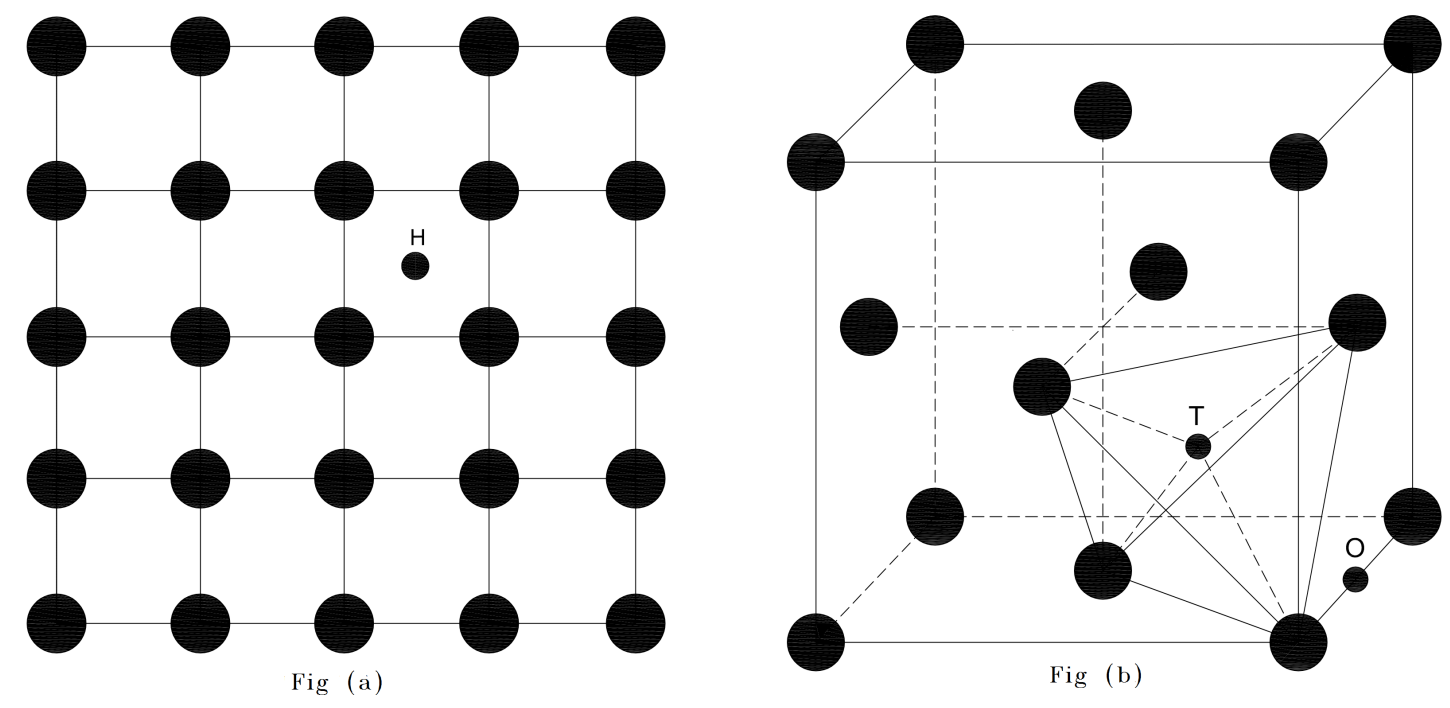

Figure 4.2: Figure (a) Schematic of a hydrogen interstitial in perfect 2D lattice; Figure (b) Hydrogen interstitial at the octahedral (O) and tetrahedral (T) sites in an FCC lattice

Table 4.2 shows the results for a single hydrogen atom in solution in the perfect bulk. It can be inferred that the hydrogen atoms are preferentially located in the 
octahedral sites as the site is approximately $0.41 \mathrm{eV}$ lower in energy than the tetrahedral site. This site preference is in agreement with previous findings of Wimmer et al. [129] and experimental observations [21].

Table 4.2: Hydrogen interstitial formation energy

\begin{tabular}{|c|c|}
\hline Interstitial site & $E_{i}[\mathrm{H}] \mathrm{eV}$ \\
\hline Octahedral & -2.19 \\
\hline Tetrahedral & -1.78 \\
\hline
\end{tabular}

Adding to our findings of interstitial hydrogen in the bulk lattice, the second stage of this work was to investigate the influence of a monovacancy upon the interstitial hydrogen. Establishing the stability of a single hydrogen atom at a monovacancy was a vital goal for us in order to proceed with our main scope of work i.e. to calculate the trapping capacity of a monovacancy (hydrogen-vacancy interaction). This calculation would aid us in identifying the possible sites of hydrogen preference within the vacancy defect.

Primarily we calculate the formation energy of the vacancy and interstitial defects completely unrelated to each other. Next we position the hydrogen atom at the sites in the vacancy. The difference of energies between these two states gives the trapping energy of the vacancy. This has been illustrated in Figure 4.3

For the monovacancy, three configurations were considered: the first two being the hydrogen atom at the octahedral and tetrahedral interstitial sites of the vacancy defect and the last being a hydrogen atom in substitution (i.e. positioned exactly at the centre of the vacancy). These configurations were studied to basically check the affinity of the hydrogen atom to the vacancy and the interstitial sites in competition. We then proceeded to fill up the vacancy with the hydrogen atoms in an efficient 

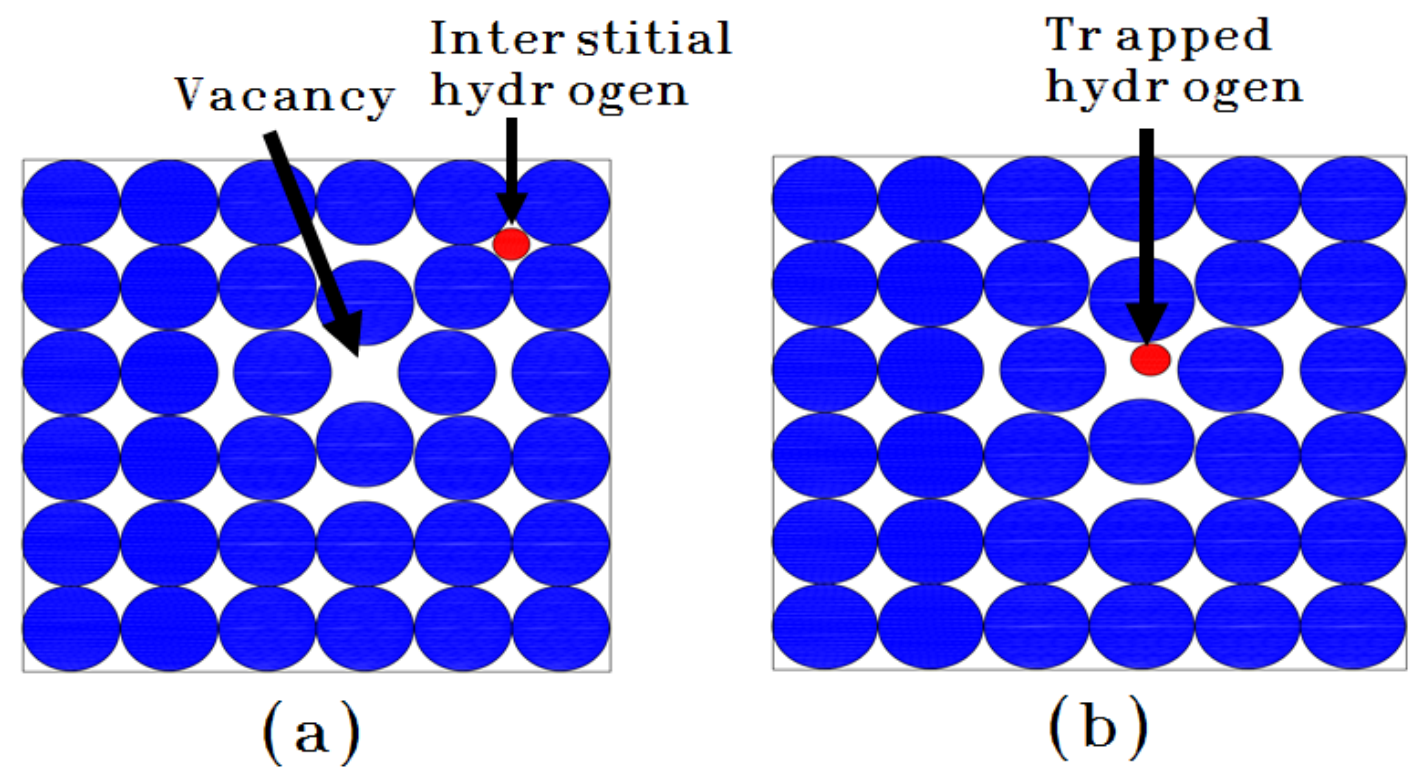

Figure 4.3: Figure (a) represents the cell with vacancy and interstitial defects unrelated each other; Figure (b) represents the trapped hydrogen interstitial at a vacancy

manner and further investigate the trapping effect i.e. whether the hydrogen atoms diffusing through the bulk Ni lattice would prefer to cluster around a vacancy or stay as interstitials. The trapping may lead to a reduction in the the defect formation energy itself [3].

The trapping or solubility energy at the vacancy is given by:

$$
E_{\text {trap }}(H)=\frac{1}{m}\left(E_{o}[(N-1) \cdot N i+m \cdot H]+E_{o}[(N-1) \cdot N i]-E_{n v}^{f}\right)-E_{i}[H]
$$

where $E_{o}[(N-1) \cdot N i+m \cdot H]$ corresponds to the energy of the system containing a vacancy and $m$ hydrogen atoms and $E_{o}[(N-1) \cdot N i]$ is the energy of the system with a single vacancy. 


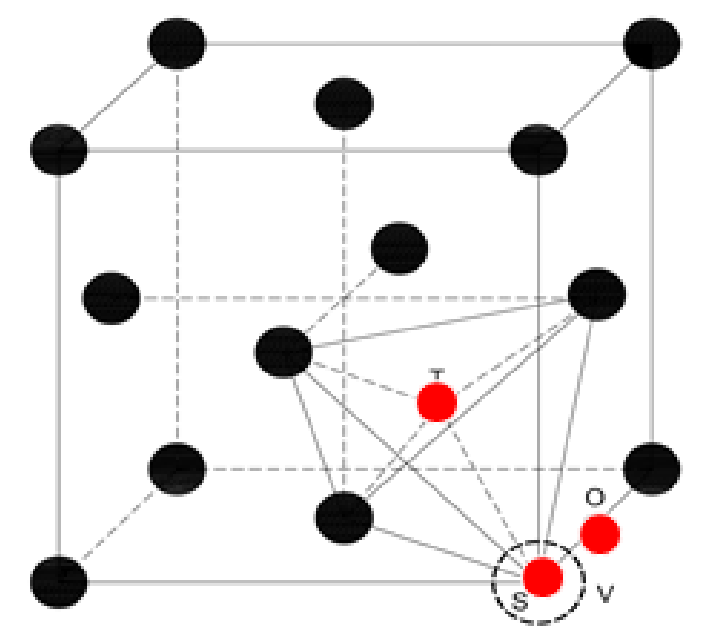

Figure 4.4: Hydrogen-vacancy (V) configurations at the substitution (S), octahedral $(\mathrm{O})$ and tetrahedral $(\mathrm{T})$ sites in an FCC lattice

The hydrogen trapping energy calculated by Equation 4.3 determines the preferential location of the interstitial hydrogen among the competing sites i.e. the center of the vacancy, the octahedral and tetrahedral positions. This is a situation where in the single vacancy concentration $C_{V}$ is greater than the hydrogen concentration $C_{H}$.

\subsubsection{Investigation of the Hydrogen-Vacancy Cluster}

On the other hand, if the $C_{H}$ is greater than $C_{V}$, the question arises as to where the excess hydrogen atoms be located, at the bulk interstitial sites or at the vacancy sites? Based on our findings from the previous section, we proceeded in simulating the hydrogen-vacancy cluster formation. This was done in order to investigate the trapping capacity of a single vacancy. Primarily, after creating a vacancy defect, the six octahedral interstitial sites of the vacancy were filled with hydrogen atoms. Energy minimization was undertaken and the trapping energy for each of the added hydrogens was calculated. A negative value of the trapping energy represented the energy gained by the system when the hydrogen atoms are trapped at a single vacancy 
site relative to be dispersed in the bulk at different independent (non-interacting) octahedral interstitial sites.

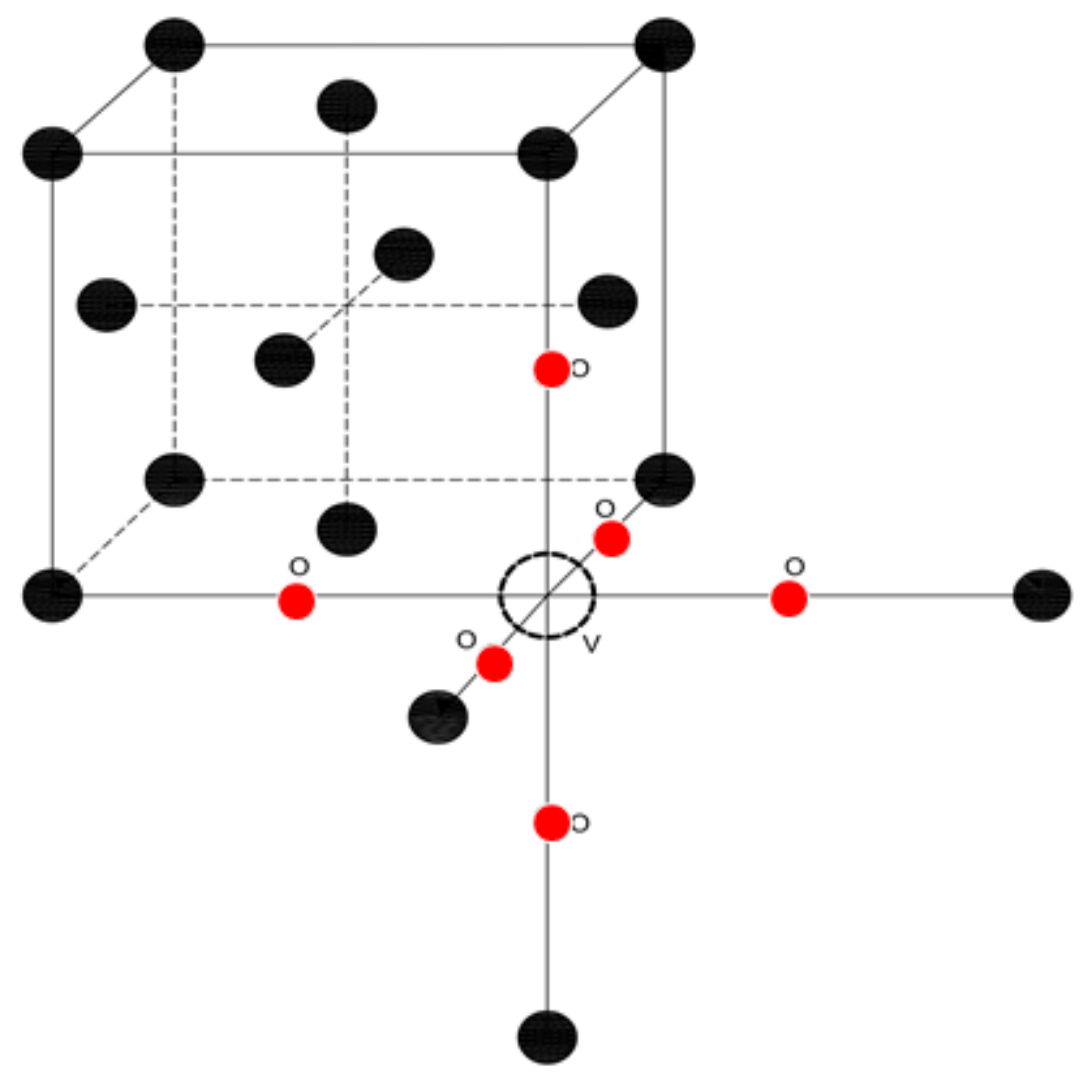

Figure 4.5: Schematic representation of a hydrogen-vacancy cluster

The Figure 4.5 indicates the environment of a vacancy $(V)$ in $\mathrm{Ni}$ with the hydrogen atoms (in red) located at the octahedral sites $(O)$. Once this operation was carried out, we had two options to pursue. One to continue filling the tetrahedral interstitial sites of the monovacancy and second to study the trapping effect when the hydrogen atoms were filled in the octahedral sites of the first-nearest-neighbour Ni atoms next to the vacancy. The motivation for pursuing this step was the result of the study by 
Gang et al. [3], wherein they perform similar calculations using DFT on first-nearestneighbour $\mathrm{Al}$ atoms. The results and analysis of this exercise have been covered in detail in Chapter 5.

Initially during the course of our study, we limited the computation to be performed on a simulation cell of size $5 \times 5 \times 5$ with $500 \mathrm{Ni}$ atoms. From the literature we did notice that most of the studies performed to evaluate the hydrogen-vacancy interactions were carried out using DFT [3], [28], [62]. DFT limits the size of the cell being studied to a few atoms. Using empirical potentials we can overcome this limitation and we could also evaluate if varying cell sizes can have an influence upon the computed trapping energy of a vacancy. Essentially, as we already know, a defect free crystal is infinite in nature and impossible to create in reality. By sequentially increasing the size of the simulation cell we tried to imitate partially a real crystal by, say, for example creating a large simulation cell of size $15 \times 15 \times 15$ containing about 13500 atoms. This study involving a step by step increase in cell size was done for range from $3 \times 3 \times 3$ to $15 \times 15 \times 15$ for $m$-hydrogen atoms around one vacancy. From

this study we could arrive at the difference in trapping energy of a vacancy between the smallest and largest cell sizes, and study the degree of energy variation. From this we can conclude whether a small size cell such as the ones used in DFT is robust enough to provide us with the correct result.

\subsubsection{Hydrogen-Vacancy Complex Migration}

At this point, we were convinced of the stability of the hydrogen-vacancy complex. The terminal scope of our study was to research the effect of hydrogen on the vacancy mobility. The stable hydrogen concentration around the point defect provided a configuration which we could subject to loads and investigate the vacancy migration pathway. 
The size of the cell spanned 10 lattice units in each direction $(10 \times 10 \times 10)$. Two configurations of the cell were considered: First a relaxed configuration with one vacancy, $3999 \mathrm{Ni}$ atoms and 12 interstitial hydrogen atoms clustered around the point defect; and second with $3999 \mathrm{Ni}$ atoms and a single vacancy with no hydrogen atoms, again in the relaxed configuration. Both the cells were subjected to tensile and compressive pressures. We were primarily interested in the results for the pressure range of $20 \mathrm{MPa}$ to $10000 \mathrm{MPa}$; but extended the range over the course of our study to evaluate the limit of the EAM potential we employed in this research.

The cells were individually subjected to a tensile-hydrostatic stress range of 20 $\mathrm{MPa}$ to $25000 \mathrm{MPa}$. The compressive load range varied from $20 \mathrm{MPa}$ to $65000 \mathrm{MPa}$. The upper limit of the load ranges were set by the EAM potential as any values beyond these upper limits, the atoms were either extremely close or distant from each other to have any kind of interaction based on the parameters that were fit into the potential during its development.

The primary energy minimization was carried out in LAMMPS which was followed by straining the cells by varying the lattice constant. ART was then applied successively to every cell until the configuration reached a stable energy. The activation energy for vacancy migration at every loaded state was thereby calculated. The results from this stage have been discussed in Section 5.4 of Chapter 5 . 


\section{Chapter 5}

\section{Results and Analysis}

The simulations were carried out in three stages; In the first stage equilibrium parameters of bulk $\mathrm{Ni}$ were calculated in order to verify the selection of the Ni-Al-H EAM potential for this study, the energetics behind the hydrogen-vacancy complex was investigated in the second stage and in the last stage, the diffusion of the vacancy under the influence of the interstitial hydrogen atoms and pressure were researched. The results of them are presented in this chapter together with discussions. The methodology details and some important preliminary findings have been already discussed in Chapter 4.

\subsection{Verification of the Bulk Properties of Defect Free Nickel}

The properties of the bulk material will have considerable influence upon the behaviour of a particular material. The starting point of any investigation is to determine the stability of the bulk crystal by calculating the theoretical lattice parameter and cohesive energy i.e. the properties of defect free nickel and comparing the same 
with the literature. The detailed procedure can be found in Section 4.1.2. The cohesive energy, defined as the energy required to separate the atoms composing a solid, varies with the distance between the atoms of a solid (unit cell volume) and reaches a minimum. This stage is termed as the equilibrium cohesive energy, i.e. the point where the pressure is zero.

The total energy curve as a function of atomic volume of nickel (refer Figure 5.1) was generated, allowing the estimation of the equilibrium parameters. The volume corresponding to the minimum energy identifies the equilibrium lattice parameter. The lattice parameter and the cohesive energy per atom for the Ni crystal were directly estimated from the Figure 5.1.

Table 5.1: The properties of bulk nickel: lattice parameter and cohesive energy per atom

\begin{tabular}{|c|c|}
\hline \multicolumn{2}{|c|}{ Parameter } \\
\hline Lattice parameter & $3.52 \AA$ \\
\hline Cohesive energy per atom & $4.45 \mathrm{eV}$ \\
\hline
\end{tabular}

Table 5.1 shows the properties of defect free nickel by implementing the Ni-AlH EAM potential. They are in agreement with theoretical values fit into the EAM potential [1] and with the experimental literature [130] [131]. This step verified the robustness of the $\mathrm{Ni}-\mathrm{Ni}$ interaction fit into the potential and justified its selection for this study. With this conclusion we could proceed in using the parameters fit into the potential (refer Table 4.1) in our further calculations as described in Section 4.1.3. 


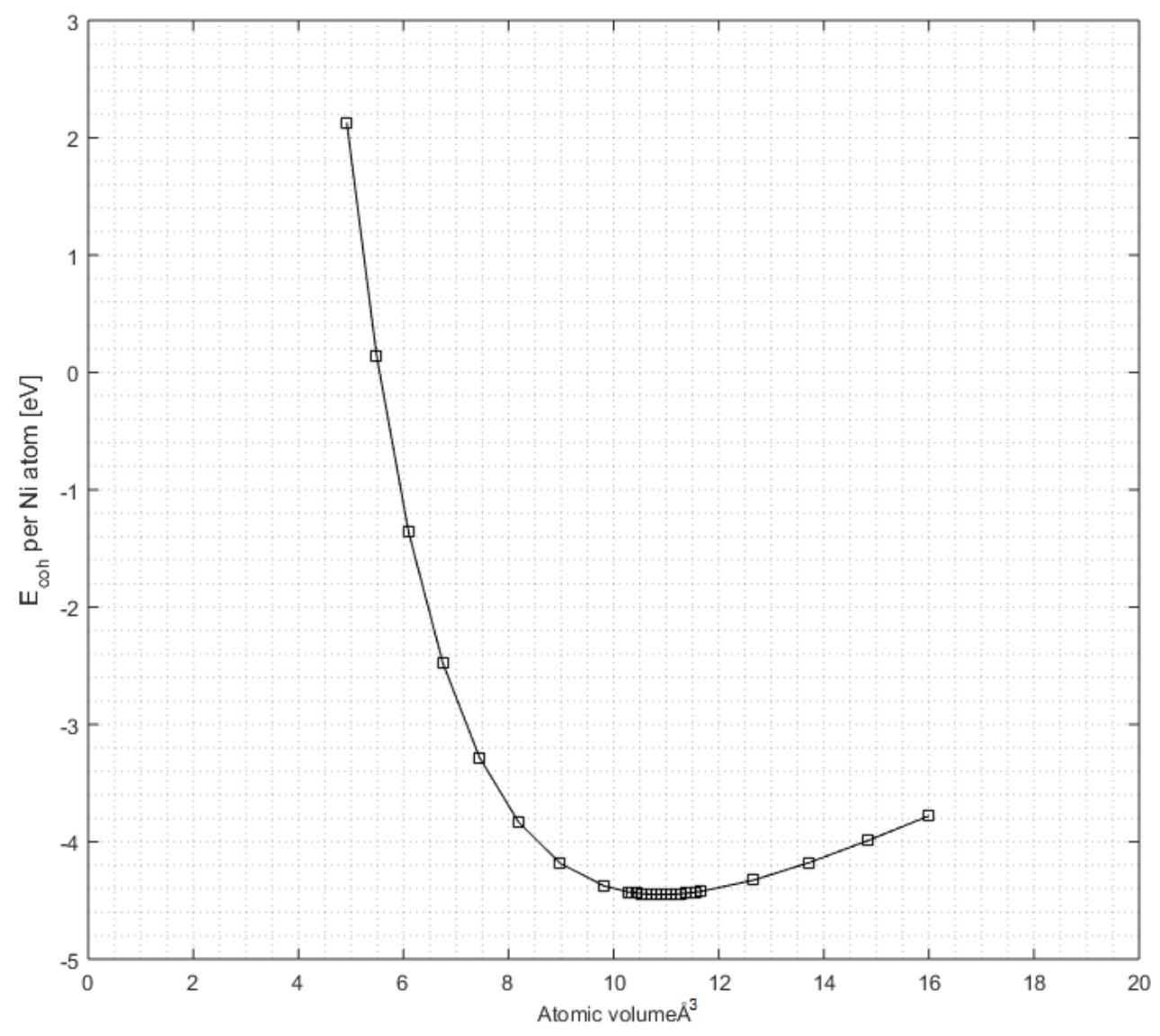

Figure 5.1: The total energy curve of nickel as a function of atomic volume

\subsection{Interactions between Hydrogen and a Mono- vacancy}

In our study, we try to understand first the hydrogen-vacancy interactions in bulk nickel. For this we had to start off by studying the two point defects in question: the vacancy and the interstitial defects. The initial calculation that we performed was to find the vacancy formation energy $E_{n v}^{f}$ using Equation 4.1. This value was essential in assessing the hydrogen trapping capacity of a monovacancy and in investigating if the point defect formation energy reduces due to interaction with interstitial hydrogen [3]. 
The vacancy formation energy in bulk nickel was found to be $1.59 \mathrm{eV}$ which is in agreement with the EAM potential fitting value [1] and is close agreement with the experimental value of $1.58 \pm 0.01 \mathrm{eV}[132]$.

The hydrogen interstitial formation energy $\left(E_{i}[H]\right)$ was calculated (refer Table 4.2) in bulk nickel using the Equation 4.2. The hydrogen atoms are located preferentially in the octahedral sites with the site being $0.41 \mathrm{eV}$ lower in energy when compared to the tetrahedral position. The $E_{i}[H]$ would be implemented in separating out the trapping energy of a monovacancy in Equation 4.3 thereby eliminating the point defect formation energy whist studying the hydrogen-vacancy cluster.

Next we proceeded in evaluating the site preference of interstitial hydrogen in the presence of a monovacancy. The trapping energy $\left(E_{\text {trap }}[H]\right)$ was calculated using the Equation 4.3 and the results have been tabulated in the Table 5.2 below.

Table 5.2: Hydrogen trapping energy as per respective configurations

\begin{tabular}{|c|c|}
\hline Site & $E_{\text {trap }}[H] \mathrm{eV}$ \\
\hline Centre of the vacancy & 0.20 \\
\hline Octahedral & -0.18 \\
\hline Tetrahedral & -0.036 \\
\hline
\end{tabular}

Table 5.2 shows the trapping energy of a single hydrogen atom under the influence of a vacancy. The difference in energy between a hydrogen atom in substitution and at the octahedral site confirms that the interstitial prefers to be located at an off-center position rather than the geometric centre of the vacancy site. The study is similar to the one performed by Gang et al. [3] where they try to find the site preference of hydrogen in bulk aluminium before evaluating the trapping capacity of a vacancy. The conclusion of our study is quite similar to the ones reported by Besenbacher et al. in nickel [99], [100]. Furthermore, the octahedral 
site is approximately $0.15 \mathrm{eV}$ lower in energy than the tetrahedral site which further adds to the fact that the stable hydrogen-vacancy configuration exists when the interstitial is located at the octahedral site. These values are in good agreement with the study performed on the stability of vacancy-hydrogen clusters in nickel by Tanguy et al. [61]. Hence the lowest energy position for the hydrogen atom in the presence of a vacancy is the octahedral interstitial position.

\subsection{Trapping Effect of Multiple Hydrogen Inter- stitials at a Monovacancy}

\subsubsection{Hydrogen-Vacancy Energetics}

Having established the stability of a single hydrogen atom at a monovacancy in nickel, the emerging question was whether multiple hydrogen atoms would be stable at this defect. This phenomenon resembles a situation wherein the single vacancy concentration $\left(C_{V}\right)$ is lower than the interstitial hydrogen concentration $\left(C_{H}\right)$. The results for

$E_{\text {trap }}[H]$ as a function of $m$-hydrogen atoms in interstitial positions of a monovacancy are summarized in Figure 5.2.

The Figure 5.2 shows that it is energetically favorable for multiple hydrogen atoms (12) to be trapped at a single vacancy site relative to being dispersed at the interstitial sites as independent atoms. The first six hydrogen atoms are located in the preferential octahedral interstitial sites and the remaining 6 hydrogen atoms are located in the tetrahedral sites of the vacancy. It can be seen that the $E_{\text {trap }}[H]$ is approximately $-0.18 \mathrm{eV}$ for 6 hydrogen atoms and it rises up to $-0.095 \mathrm{eV}$ when the $7^{\text {th }}$ is trapped at the tetrahedral site; stabilizing at approximately $-0.055 \mathrm{eV}$ for 12 


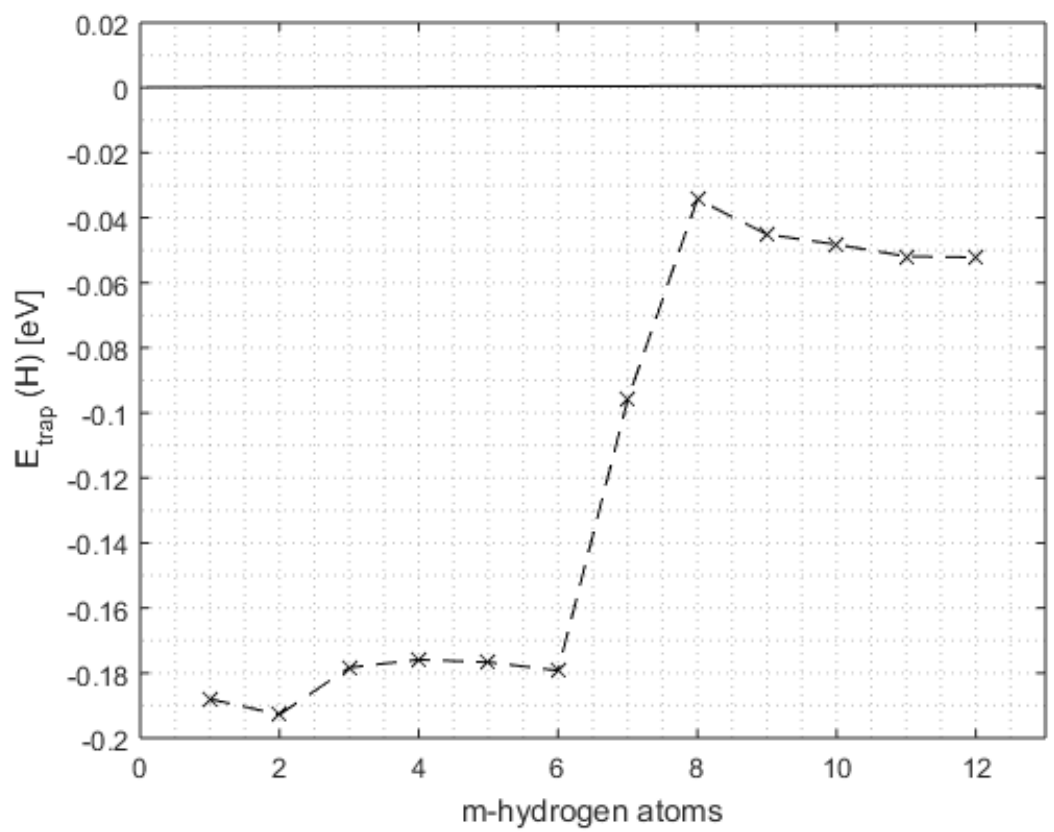

Figure 5.2: Trapping energy per hydrogen atom in $\mathrm{eV}$ as a function of the number of hydrogen atoms being trapped at the octahedral and tetrahedral sites of a vacancy

hydrogen atoms. This upward trend reconfirms our initial findings that the octahedral sites in the vacancy are preferred by interstitial hydrogen atoms in comparison with tetrahedral sites. We also observe that the tetrahedral sites do serve as trapping sites but as more hydrogen atoms are filled in the vacancy, the $E_{\text {trap }}[H]$ tends to be move towards the positive value (closer to $0 \mathrm{eV}$ ); meaning the hydrogen atoms would rather be displaced as interstitial atoms in the bulk, than being trapped by a vacancy.

\subsubsection{Hydrogen-Vacancy Energetics involving First-Nearest- Neighbor Sites}

The surge in $E_{\text {trap }}[H]$ with the trapping of the $7^{\text {th }}$ hydrogen atom in the tetrahedral site and the findings reported by Gang et al. [3] led us to investigate the trapping energetics of the monovacancy, where interstitial hydrogens are positioned in the 




Figure 5.3: Trapping energy per hydrogen atom in $\mathrm{eV}$ as a function of the number of hydrogen atoms being trapped at the octahedral sites

octahedral sites of the first-nearest atoms. The results for $E_{\text {trap }}[H]$ as a function of $m$-hydrogen atoms in octahedral interstitial positions of a vacancy and first-nearestneighbour Ni atoms of the vacancy are summarized in the Figure 5.3.

The Figure 5.3 represents a $E_{\text {trap }}[H]$ value of $-0.14 \mathrm{eV}$ for the $12^{\text {th }}$ hydrogen atom. It is observed that the hydrogen atoms would preferentially be located at octahedral sites of the first-nearest-neighbor $\mathrm{Ni}$ atoms of the vacancy rather than being located at the tetrahedral sites (in comparison to Figure 5.2). These results are similar to the trapping of up to 12 hydrogen atoms at a vacancy in $\mathrm{Al}$ [3] and twice or more the highest number of hydrogen atoms (six) that can be trapped in Fe [28]. The plot also shows that the number of hydrogen atoms trapped under the influence of the vacancy can exceed 12 .

In a FCC cell, each atom has 12 nearest-neighbors, each with six octahedral 
sites. The greater the number of hydrogen atoms, the more interstitial site combinations that need to be studied through energy minimizations. Therefore, it becomes more and more complicated to determine a true minimum. The total number of arrangements required is a function of the nearest-neighbors and their octahedral sites. Figures 5.4 and 5.5 provide an example of two of the many possible arrangements of hydrogen interstitials around the same vacancy that would need to be computed in order to find the true minima. Different arrangements lead to different trapping energies, the Figure 5.4 arrangement has a trapping energy of $-0.058 \mathrm{eV}$ and Figure 5.5 of $-0.06 \mathrm{eV}$. The true minimum can therefore only be established when all arrangement simulations are exhausted, a task that grows significantly with the number of interstitials. This is the main reason why a maximum of 12 atoms were studied in this research.

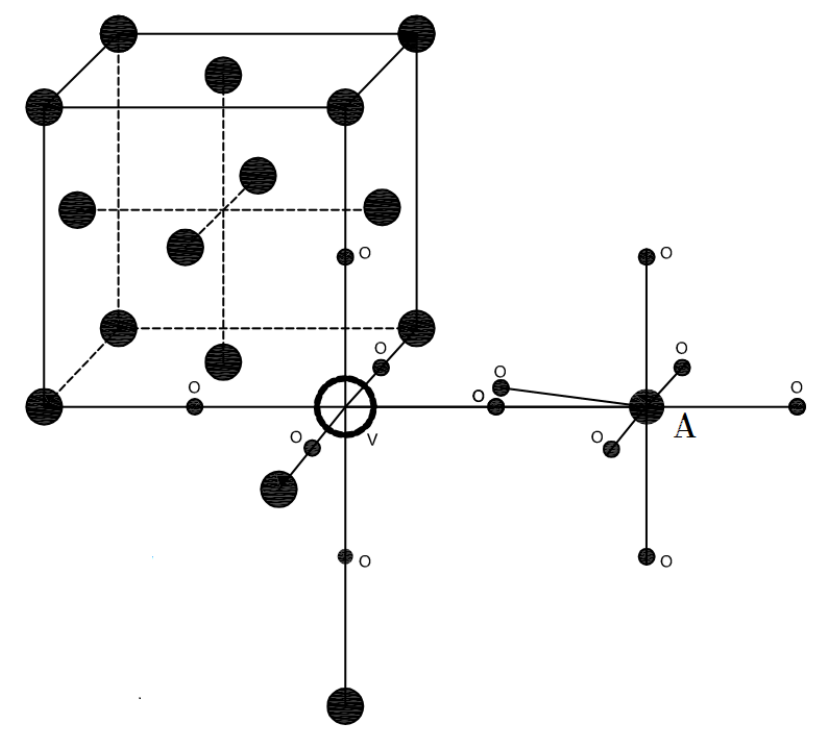

Figure 5.4: Schematic representation of hydrogen interstitials around a vacancy $(\mathrm{V})$. The figure illustrates 12 hydrogen atoms at octahedral (O) sites around the vacancy and first-nearest-neighbor $\mathrm{Ni}$ atom $\mathrm{A}$ 


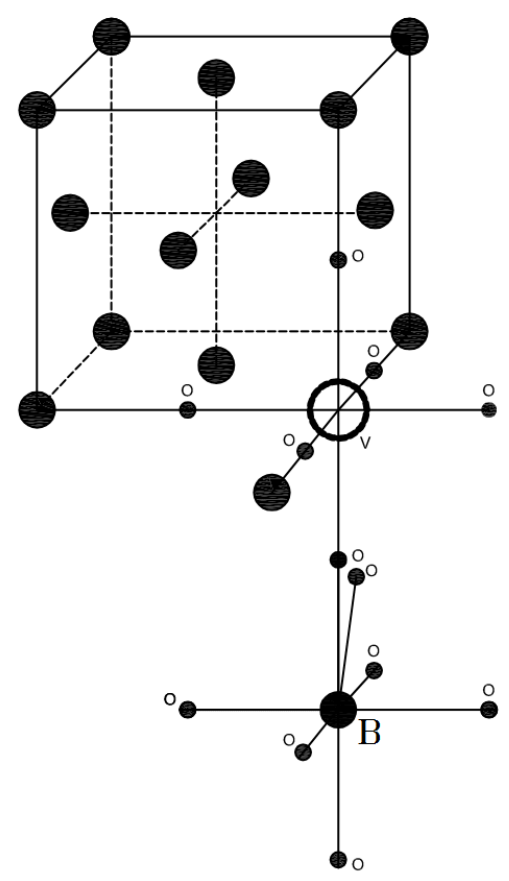

Figure 5.5: Schematic representation of hydrogen interstitials around a vacancy $(\mathrm{V})$. The figure illustrates 12 hydrogen atoms at octahedral (O) sites around the vacancy and first-nearest-neighbour Ni atom B

\subsubsection{Interstitial Hydrogen Complex Energetics}

We have brought out earlier in our study (refer Section 4.1.3, Table 4.2) that as independent interstitials, the hydrogen atoms tend to prefer the octahedral interstitial sites in the bulk lattice. But owing to our findings in the previous section, in order to validate the clustering effect of the hydrogen atoms around a single vacancy we simulated the clustering of the same number of hydrogen atoms around a $\mathrm{Ni}$ atom in the absence of a vacancy. The results for $E_{\text {trap }}[H]$ as a function of $m$-hydrogen atoms in octahedral interstitial positions clustered around a $\mathrm{Ni}$ atom and a single vacancy are summarised in the Figure 5.6.

From Figure 5.6 it is evident that the hydrogen atoms prefer to the cluster around the vacancy but also show a tendency to cluster in the defect free bulk Ni lattice. 


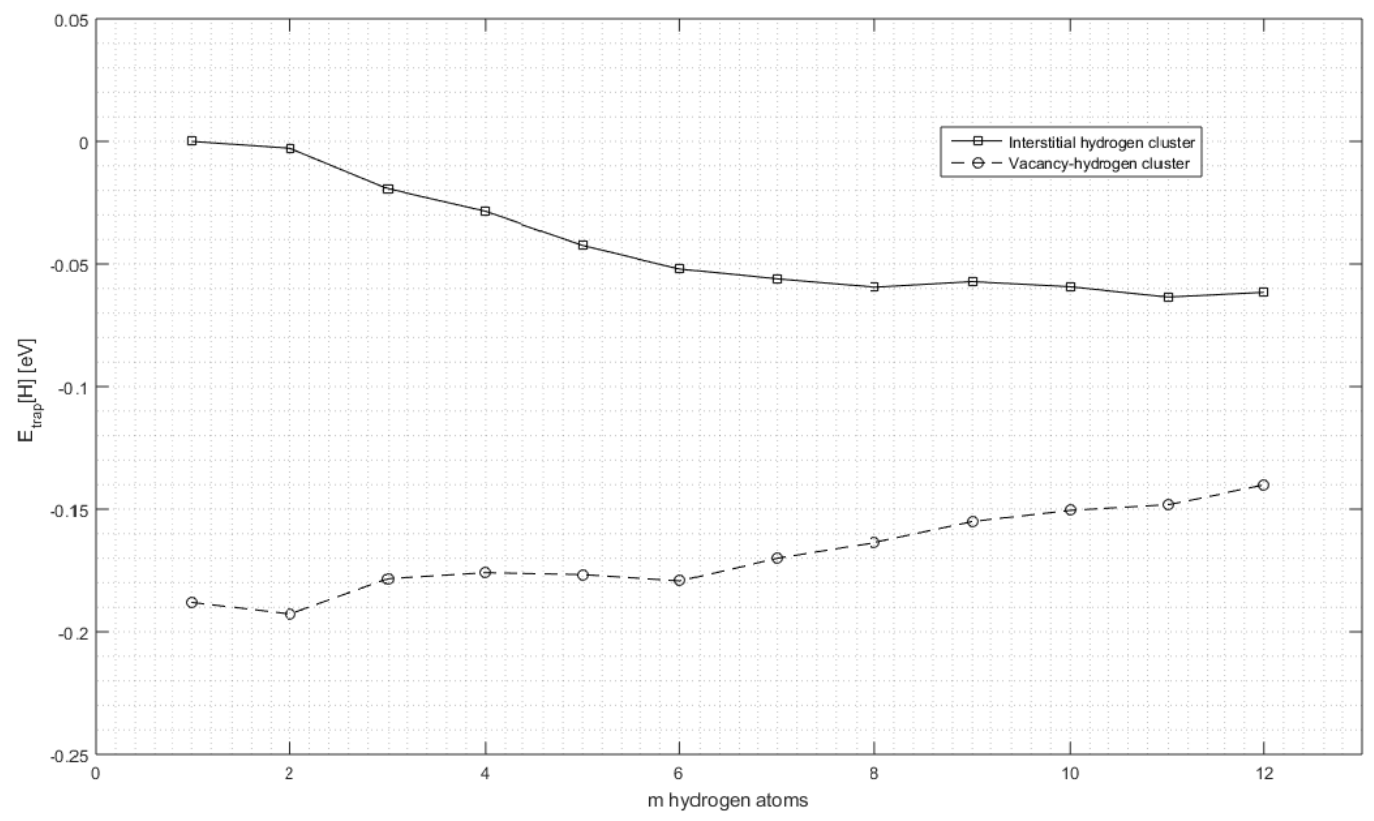

Figure 5.6: Trapping energy per hydrogen atom in $\mathrm{eV}$ as a function of the number of hydrogen atoms being trapped (in the vicinity of a $\mathrm{Ni}$ atom and a monovacancy)

The difference in the trapping energy for 12 hydrogen atoms between the two systems under study is $0.07 \mathrm{eV}$ which is significant. From this we understand that the hydrogen atoms would be present in octahedral interstitial sites in the absence of a vacancy. This study further adds weight on our findings in the previous sections and we can conclude here that the hydrogen atoms will cluster in and around the vacancy defect rather than existing as interstitials at octahedral sites in the bulk lattice.

\subsubsection{Maximum Trapping Capacity of a Monovacancy}

The findings from our previous simulations led us to investigate the maximum number of interstitial hydrogens that can be trapped by a vacancy until the $E_{\text {trap }}[H]$ value exceeds $0 \mathrm{eV}$. This study could enable us to preliminarily investigate if the vacancy has a bigger influence on the nearest-neighbour sites and to check if these findings from empirical potentials could lay the ground work for more detailed analysis of 
hydrogen-vacancy clusters using $k$-ART or $D F T$ techniques. Energy minimization simulations were undertaken wherein we continued filling in the octahedral sites of the first-nearest-neighbour $\mathrm{Ni}$ atoms of the vacancy. We did however retain the first 12 hydrogen atoms to be positioned within the octahedral and tetrahedral interstitial positions of the monovacancy based on our previous results (refer Section 5.3.1). The outcome for $E_{\text {trap }}[H]$ as a function of $m$-hydrogen atoms (exceeding 12 ) in a combination of interstitial positions within the monovacancy and first-nearest-neighbour $\mathrm{Ni}$ atoms are summarized in the Figure 5.7.

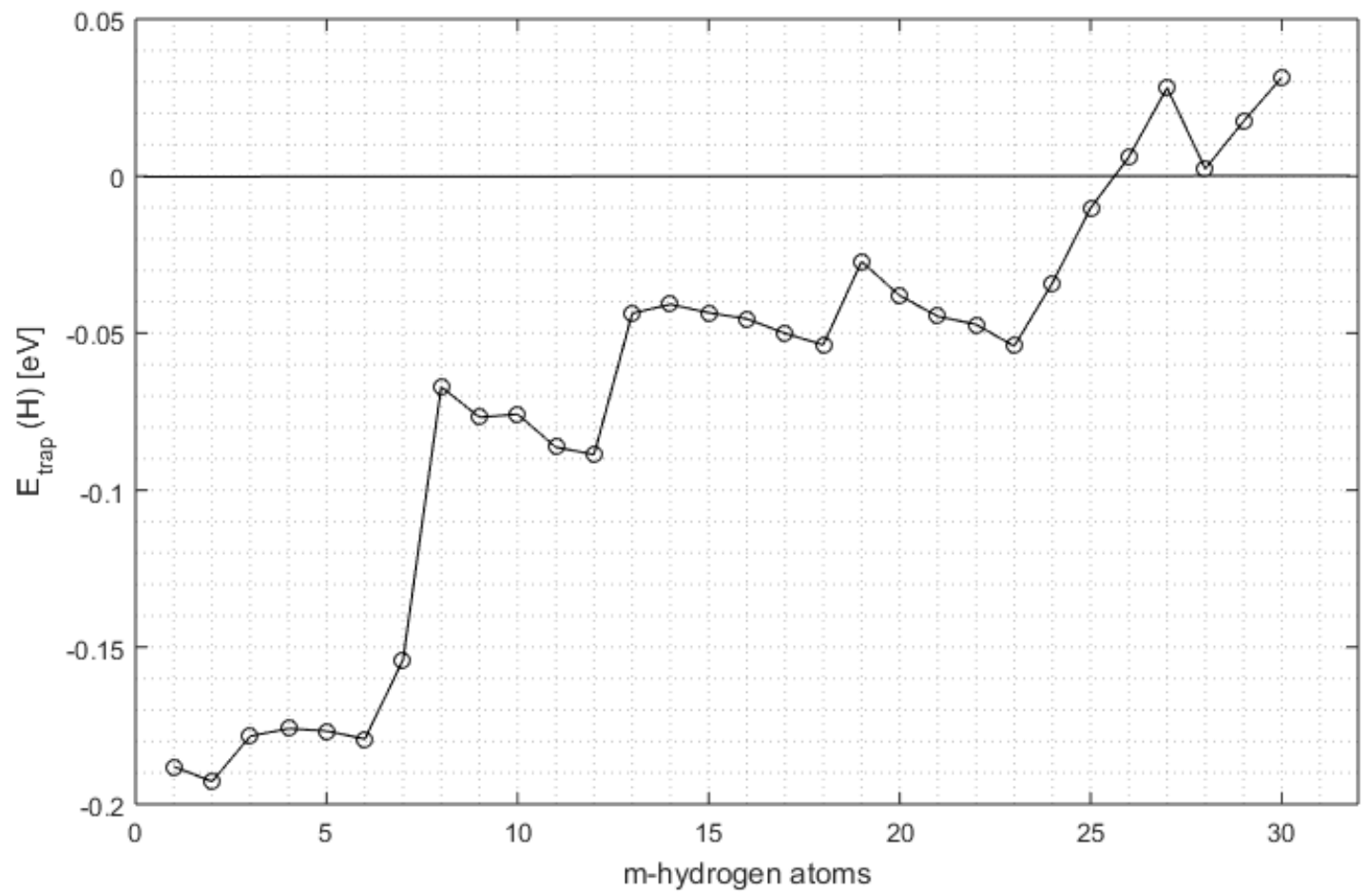

Figure 5.7: Trapping energy per hydrogen atom in $\mathrm{eV}$ as a function of the number of hydrogen atoms (exceeding 12) being trapped in and around a vacancy

The Figure 5.7 shows that up to 25 hydrogen atoms can be trapped in and around the vacancy, beyond which they prefer to be located in octahedral interstitial positions of $\mathrm{Ni}$ atoms in the bulk lattice. The vacancy does exert a trapping effect on the 
interstitial sites beyond its volume. The plot has significant high and low points as a result of the high number of octahedral site combinations (six per $\mathrm{Ni}$ atom) that exist at each first-nearest-neighbour atoms of the monovacancy. The result from this particular study is an unrefined estimate of the trapping capacity. But it throws light on a situation where we observe the potential for hydride formation around the vacancy when the $C_{V}<C_{H}$, which might lead to embrittlement and ductile failure of the metal. This kind of situation may arise when nickel is being charged with hydrogen atoms and high diffusion of the interstitial atoms through the bulk occurs in the absence of bigger defects like voids or dislocations.

\subsubsection{Hydrogen-Vacancy Interaction based on Simulation Cell Size}

The hydrogen-vacancy complex interaction was primarily investigated on a simulation cell of size $5 \times 5 \times 5$ containing $499 \mathrm{Ni}$ atoms (refer Section 4.1.4). Once we were convinced about the trapping capacity of a monovacancy for 12 hydrogen atoms along with their locations, we simulated the same set of energy minimization techniques and calculations on range of cell sizes from $3 \times 3 \times 3$ with 107 atoms to $15 \times 15 \times 15$ with 13499 atoms, with all the cells containing a single vacancy. This study was undertaken with large cell sizes to simulate the effect of a real crystal, ideally infinite in nature but impossible to create. The effect of cell size upon the trapping energy is evaluated here. The results of $E_{\text {trap }}[H]$ as a function of hydrogen atoms at interstitial sites around a vacancy for the cell range implemented in this study is summarized in the Figure 5.8.

The trend for all the cell sizes in Figure 5.8 is consistent with each other when the first 6 hydrogen atoms are located in the octahedral sites of the monovacancy. 
However for the $12^{\text {th }}$ hydrogen atom the difference in the trapping energy between the $3 \times 3 \times 3$ and $15 \times 15 \times 15$ cell sizes is approximately $0.0145 \mathrm{eV}$. From the figure, it is evident that the trapping energy is essentially converged at cell size $6 \times 6 \times 6$ beyond which the variation in trapping energy is minor between subsequent larger cell sizes. The Figure 5.8 thereby illustrates a convergence study where we consider the $15 \times 15 \times 15$ cell size to represent a infinite cell wherein the interaction of the defect being researched under periodic boundary conditions is negligible. The percent error of the $3 \times 3 \times 3$ cell in comparison to the $15 \times 15 \times 15$ cell is approximately $10 \%$, which is a error estimate of small sized DFT calculations if used to calculate the trapping effect. The equilibrium density of the vacancy defect in $\mathrm{Ni}$ at ambient conditions is much lower than the lowest case studied here in the $15 \times 15 \times 15$ cell i.e. one defect for $13500 \mathrm{Ni}$ atoms.

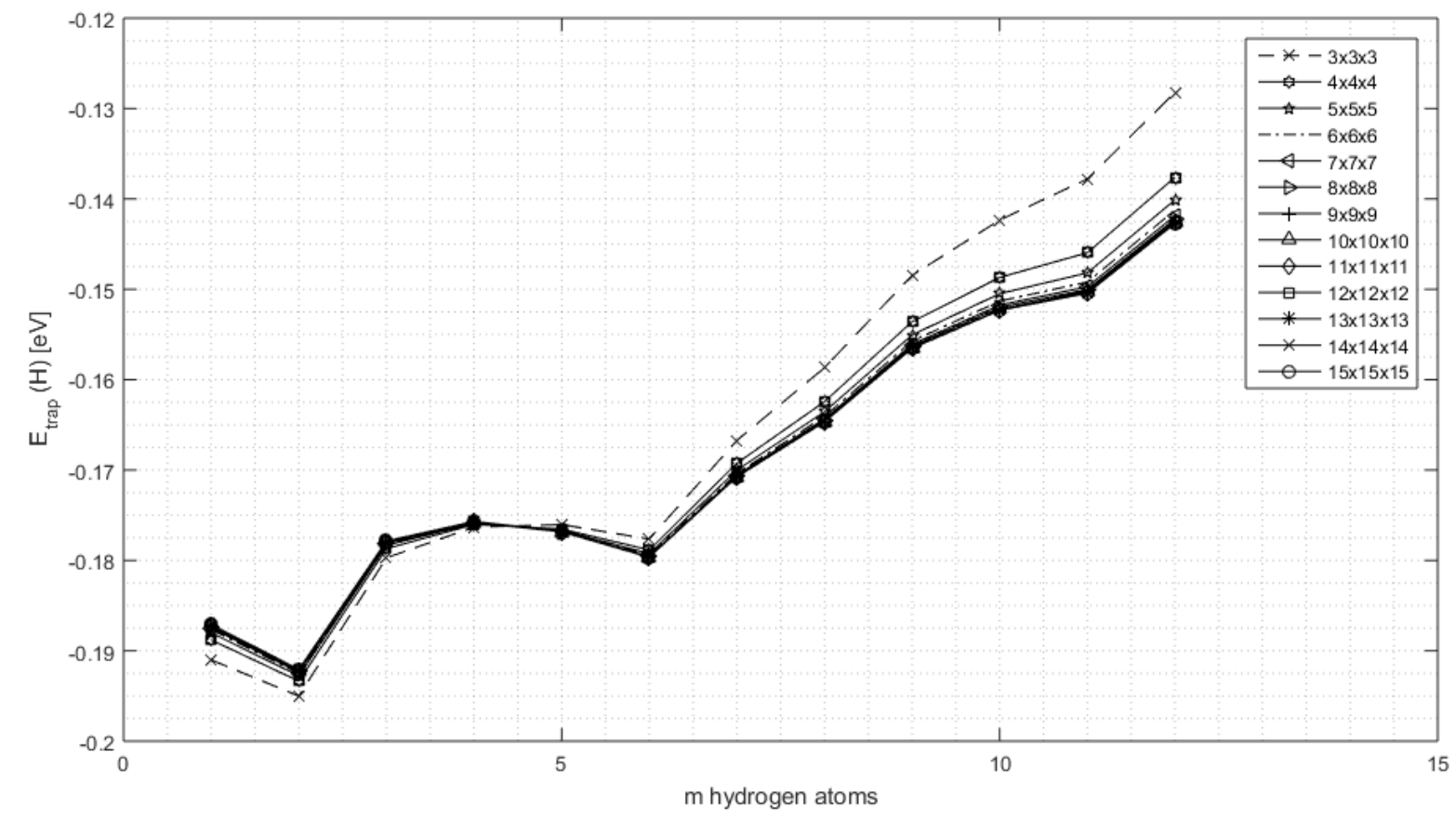

Figure 5.8: Summary of trapping energy as a function of a trapped hydrogen atoms for a range of cell sizes 
The graphical representation of trapping energy for 1 to 12 hydrogen atoms as function of system size has been covered in Appendix A. All the plots effectively prove that the empirical potential used in this study has significant effect upon the trapping energy for cell sizes having up to 4000 atoms. The visible effect tends to become very minor and aims to settle down by taking a linear profile with systems containing large number of atoms, say in the range between 4000 to 13500 atoms. However, between the smallest and the largest cell sizes, the difference in trapping energy for the range of hydrogen atoms 1 to 12 is significant. This effect is not really accounted in DFT studies due to their inherent limitations. Hence empirical potentials have an upper hand while accounting large cell sizes and are able to capture this effect while studying defects.

\subsection{Effect of Pressure on the Hydrogen-Vacancy Complex}

\subsubsection{Activation Energy for Vacancy Migration}

Atomistic simulations of the effects of hydrogen on vacancy mobility and pile-ups were performed to investigate possible nanoscale mechanisms for hydrogen embrittlement by hydrogen enhanced local plasticity. We first examined the pure Ni system, which served as a reference for the behavior in the Ni-hydrogen system. The hydrogenvacancy cluster serves as a system pre-charged with hydrogen, where the interstitial hydrogen atoms form highly concentrated clouds around the monovacancy after relaxation. Song and Curtin [4] reported that hydrogen clouds around dislocations in alpha iron tend to follow the mobile defects under strained conditions and exert resistance to the defect motion. Based on the findings from this study, we researched 
the effect of tensile and compressive loads on the mobility of the hydrogen-vacancy complex. The detailed methodology can be found in Section 4.1.5 of Chapter 4 .

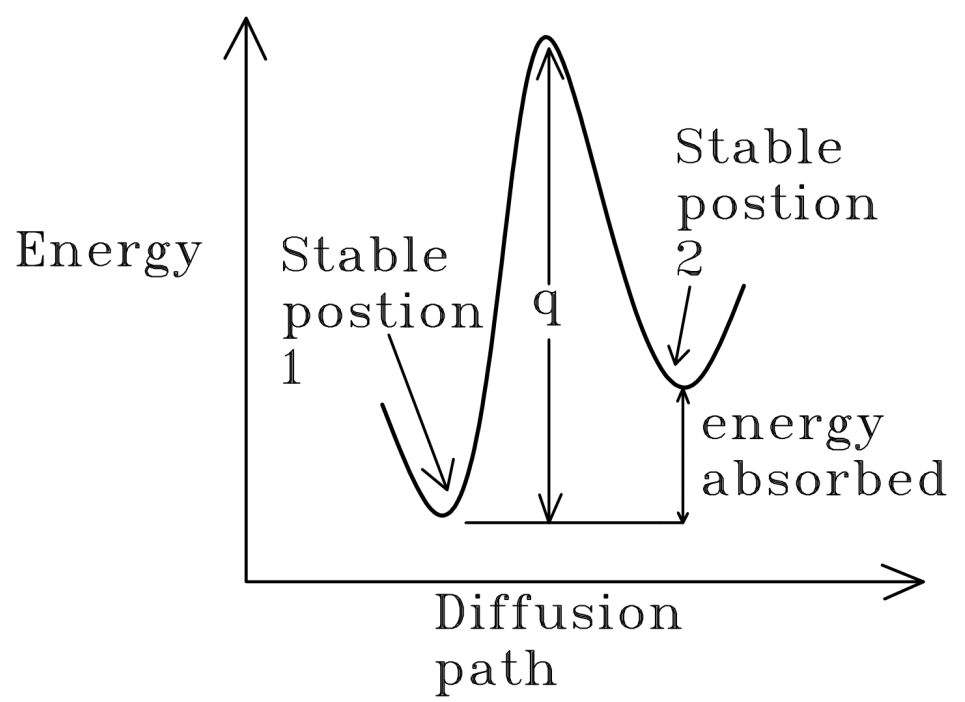

Figure 5.9: Illustration of activation energy for vacancy migration under the infuence of a hydrogen cloud

The Figure 5.9 illustrates the energetics behind this study. The hydrogen-vacancy complex at a relaxed minimum (stable position 1) has to overcome the activation energy $(q)$ to diffuse and arrive at a new minimum (stable position 2). Post analysis of the simulation data, we noticed that the new configuration exists at a higher energy state.

The plot of activation energy required for vacancy migration as a function of pressure on the simulation cells is shown in Figure 5.10, with the tensile pressures shown as negative and compressive pressures as positive.

The first thing that is evident from the Figure 5.10 is that the activation energy is high for compressive loads because the energy barrier to overcome for diffusion or migration of the vacancy is high. At tensile loads, the energy barrier is lower, due to creation of space by increase in lattice constant between atoms. Also evident from 


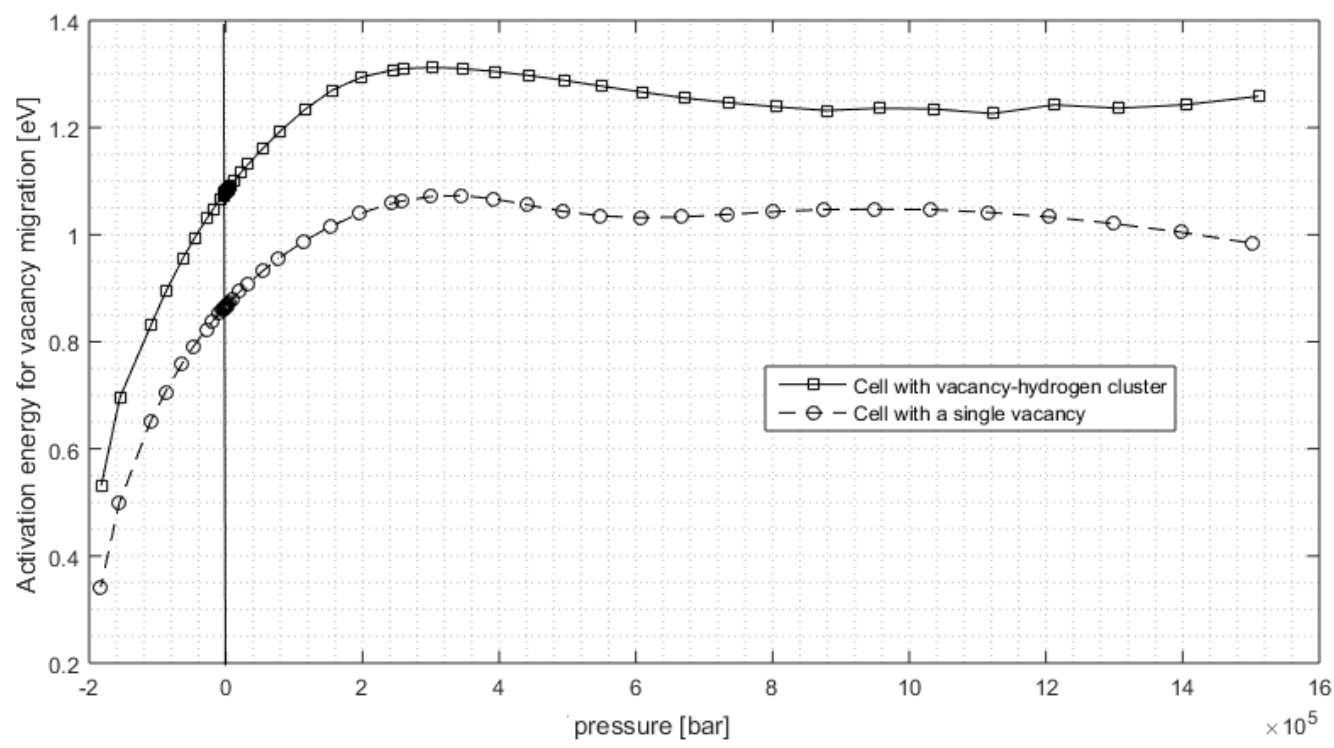

Figure 5.10: Comparison of activation energy for vacancy migration as function of pressure for a hydrogen-vacancy complex and a single vacancy

the curve is that the vacancy is capable of migrating with a lower activation energy in comparison to the migration of the vacancy under the infuence of a hydrogen complex. This implies, the hydrogen cloud around the vacancy offers some amount of drag against the vacancy diffusion leading to a higher activation energy at this state. This result is in agreement with similar findings by Song and Curtin [4] which reported drag exerted by the hydrogen cloud around dislocation defects. The curves also indicate a slight variation in energy at higher compressive loads. We estimate this may be a result of the fit of parameters structured within the EAM potential. The maximum difference between the activation energies of the two combinations considered in this study is approximately $0.3 \mathrm{eV}$, representing an estimate of the effective drag on vacancies due to the hydrogen clusters. 


\subsubsection{Trapping Energy}

The final part of this study is the investigation of the effect of pressure upon the trapping energy of the vacancy. Two states were examined here. First, the hydrogenvacancy complex at the original position and second the complex at the new position after vacancy migration. The Figure 5.11 is a summary of the trapping energy as a function of pressure.

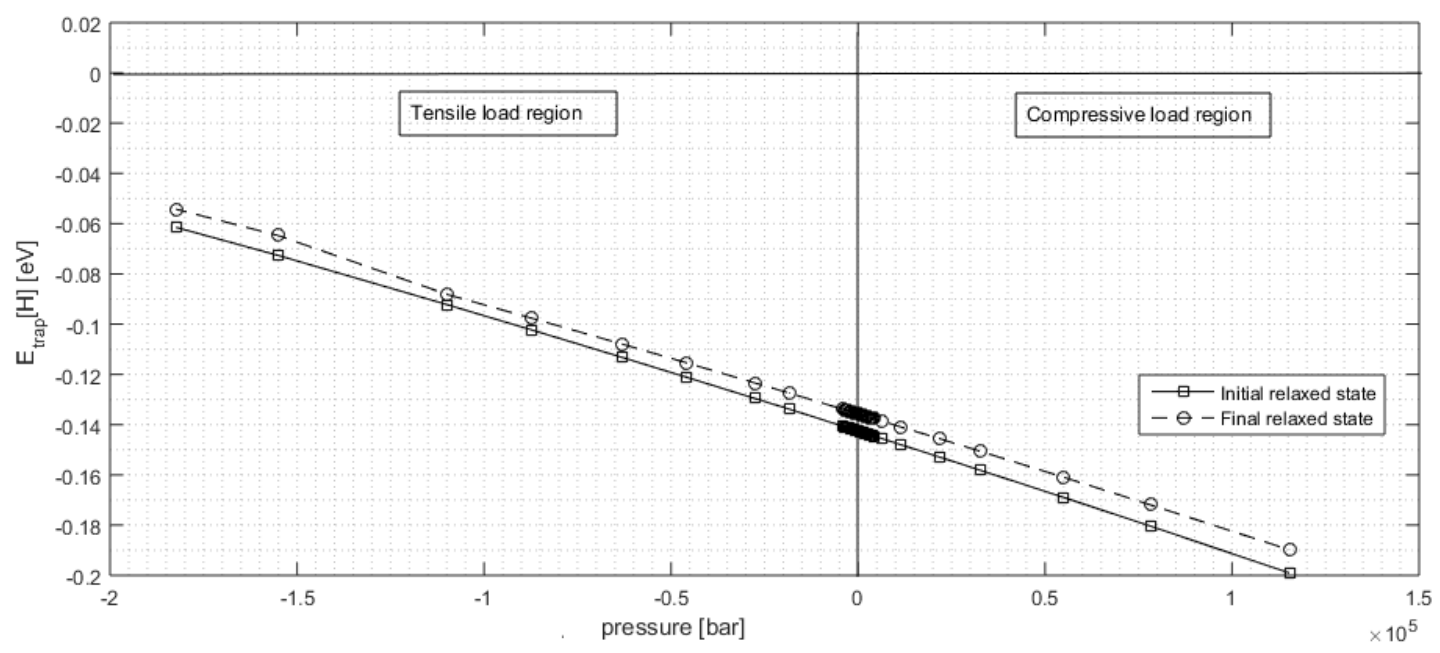

Figure 5.11: Comparison of trapping energy of a vacancy as a function of pressure for a hydrogen-vacancy complex

In Figure 5.11, it can be observed that the vacancy after migrating to a new position still has the ability to trap the interstitial hydrogen atoms i.e. the trapping energy is well below the zero energy line. This implies that with the increase in the local atom strain, the hydrogen cloud around the vacancy does not move towards to interstitial sites of the $\mathrm{Ni}$ atoms and prefers to be positioned with the vacancy. The difference in the trapping energy capacity under tensile load conditions is approximately $0.007 \mathrm{eV}$ and under compressive loads is approximately $0.01 \mathrm{eV}$. The vacancy at the new position however contains a slightly lower trapping capacity owing to the energy lost to overcome the drag exerted by the hydrogen cloud (refer Figure 
5.9. From our results, we have shown that the trapping energy is strongly negative. The hydrogen-vacancy complex is stable under strained conditions post migration. This implied that the interstitial hydrogen atoms will follow the vacancy under the conditions studied instead of being dissipated into the lattice configuration. The conclusion being the vacancy retains its ability to remain as a effective trapping zone under loaded conditions for interstitial hydrogen. 


\section{Chapter 6}

\section{Conclusions and Future Recommendations}

Atomistic modeling and simulations are gaining popularity among researchers and scientists for investigating material properties with a view of enhancing their desirability in many engineering applications. The purpose of this thesis has been to give a better understanding of the underlying mechanisms of hydrogen interstitial trapping in nickel at the vacancy point defect which may lead to hydrogen embrittlement. Configurations were investigated through energy minimization using LAMMPS and the ART feature in k-ART by implementing the Ni-Al-H embedded atom method potential. Several important bulk material properties for nickel are reproduced that

agree well with literature. The vacancy formation energy agrees well with previously published computational and experimental work. Formation energies for various interstitial configurations were also calculated which show that the octahedral site is the prevalent occupation site in the lattice configuration. Under the influence of the vacancy, the octahedral site retains its dominant state over the substitutional site. Twelve or more hydrogen atoms can be trapped at a single vacancy site. The vacancy has an influence over the octahedral sites of its first-nearest-neighbour $\mathrm{Ni}$ atoms and over 20 hydrogen atoms can be trapped under this influence. This may lead to the formation of a hydride under high local concentrations of hydrogen. 
We have shown that the vacancy is capable of trapping multiple hydrogen atoms with local interstitial concentrations much higher than the average bulk value. The strong binding between the hydrogen and vacancy defect together with the stability and mobility of the cluster may lead to the formation of hydrogen enriched microvoids by the coalescence of vacancies with trapped hydrogens. The lattice mobility of hydrogen atoms is also enhanced due to this multiple trapping at a single vacancy. This notable trapping at vacancies can contribute to a chain of events by which a substantial increase of local hydrogen concentration may occur without significant pile up at bulk interstitial sites. These vacancy based mechanisms may lead to loss in ductility as it is generally believed that hydrogen induced embrittlement in metals is through plastic rupture.

Our study on the effect of cell size upon trapping energy has shown considerable variation between the smallest and largest cell size studied (up to $0.014 \mathrm{eV}$ ); which indicates empirical potentials are capable of capturing the complete mechanics at the atomistic level, while DFT may be less accurate due to the limitation of small system sizes. Cell sizes containing upto 13500 atoms were investigated in this research, showing that the results had essentially converged to a value independent of the system size.

We have investigated the possible role of hydrogen in influencing vacancy mobility and pile-up in $\mathrm{Ni}$, as a means of understanding the whether all these vacancy-based mechanisms contribute to the hydrogen embrittlement. Our atomistic simulations show that hydrogen interstitials can form clusters around the moving vacancy, causing solute drag and thereby resisting rather than enhancing vacancy migration. Adding to this, our simulations show that the hydrogen-vacancy cluster, under the applied pressures remains stable.

Future work should first include the calculation of the maximum trapping 
capacity of a single vacancy, wherein detailed hydrogen configurations need to be studied accounting the large number of first-nearest-neighbour octahedral sites around the vacancy. The interaction of two or more hydrogen enriched vacancies amongst themselves would be interesting to investigate if they form micro-voids. The binding between the clusters and dislocation cores in the bulk might lead to a better understanding of hydrogen embrittlement through plastic rupture. Whether the hydrogen cloud provides measurable shielding of vacancy interaction or against other defects is another point to research. 


\section{List of References}

[1] J. E. Angelo, N. R. Moody, and M. I. Baskes, "Trapping of hydrogen to lattice defects in nickel," Modelling and Simulation in Materials Science and Engineering, vol. 3, no. 3, pp. 289-307, 1995.

[2] E. B. Tadmor and R. E. Miller, Modeling materials: continuum, atomistic and multiscale techniques. Cambridge University Press, 2011.

[3] G. Lu and E. Kaxiras, "Hydrogen embrittlement of aluminum: the crucial role of vacancies," Physical review letters, vol. 94, no. 15, p. 155501, 2005.

[4] J. Song and W. Curtin, "Mechanisms of hydrogen-enhanced localized plasticity: an atomistic study using $\alpha$-fe as a model system," Acta Materialia, vol. 68, pp. 61-69, 2014.

[5] J. W. Gibbs, Elementary principles in statistical mechanics. Courier Corporation, 2014.

[6] J. W. Gibbs, Scientific Papers: Thermodynamics, vol. 1. Dover Publications, 1961.

[7] R. J. Haüy, Traité de minéralogie, vol. 1. chez Louis, 1801.

[8] W. D. Callister and D. G. Rethwisch, Materials science and engineering: an introduction, vol. 7. Wiley New York, 2007.

[9] T. Sinno and R. A. Brown, "Modeling microdefect formation in czochralski silicon," Journal of The Electrochemical Society, vol. 146, no. 6, pp. 2300-2312, 1999.

[10] J. Joannopoulos and M. L. Cohen, "Electronic properties of complex crystalline and amorphous phases of ge and si. ii. band structure and optical properties," Physical Review B, vol. 8, no. 6, p. 2733, 1973. 
[11] H. Maryon, R. Organ, O. Ellis, R. Brick, R. Sneyers, E. Herzfeld, and F. Naumann, "Early near eastern steel swords," American Journal of Archaeology, vol. 65, no. 2, pp. 173-184, 1961.

[12] C. T. Sims, N. S. Stoloff, and W. C. Hagel, "superalloys ii," 1987.

[13] A. Fasching, D. Norwich, T. Geiser, and G. W. Paul, "An evaluation of a nitico alloy and its suitability for medical device applications," Journal of Materials Engineering and Performance, vol. 20, no. 4, pp. 641-645, 2011.

[14] S. Banerjee and P. Mukhopadhyay, Phase transformations: examples from titanium and zirconium alloys, vol. 12. Elsevier, 2010.

[15] S. Dushman, Scientific foundations of vacuum technique. New York: Wiley, 2d ed., 1962.

[16] R. McLellan and C. G. Harkins, "Hydrogen interactions with metals," Materials Science and Engineering, vol. 18, no. 1, pp. 5-35, 1975.

[17] T. Graham, "On the absorption and dialytic separation of gases by colloid septa," Philosophical transactions of the Royal Society of London, vol. 156, pp. 399-439, 1866.

[18] Y. Tang and J. A. El-Awady, "Atomistic simulations of the interactions of hydrogen with dislocations in fcc metals," Physical Review B (Condensed Matter and Materials Physics), vol. 86, no. 17, 2012.

[19] R. Oriani, "Hydrogen degradation of ferrous alloys," Noyes Publications, Mill Rd. at Grand Ave, Park Ridge, New Jersey 7656, USA, 1985. 886, 1985.

[20] H. Birnbaum, "Hydrogen embrittlement," Encyclopedia of Materials Science and Engineering, 1986.

[21] Y. Fukai, The metal-hydrogen system: basic bulk properties, vol. 21. Springer Science \& Business Media, 2006.

[22] A. Nakano, M. E. Bachlechner, T. J. Campbell, R. K. Kalia, A. Omeltchenko, K. Tsuruta, P. Vashishta, S. Ogata, I. Ebbsjö, and A. Madhukar, "Atomistic simulation of nanostructured materials," IEEE Computational Science $\&$ Engineering, vol. 5, no. 4, pp. 68-78, 1998. 
[23] N. D. Hine, P. D. Haynes, A. A. Mostofi, C.-K. Skylaris, and M. C. Payne, "Linear-scaling density-functional theory with tens of thousands of atoms: Expanding the scope and scale of calculations with onetep," Computer Physics Communications, vol. 180, no. 7, pp. 1041-1053, 2009.

[24] A. F. Voter, "Interatomic potentials for atomistic simulations.," MRS bulletin, vol. 21, no. 02, pp. 17-19, 1996.

[25] R. E. Miller, On the generalization of continuum models to include atomistic features. 1998.

[26] M. S. Daw and M. I. Baskes, "Embedded-atom method: Derivation and application to impurities, surfaces, and other defects in metals," Physical Review B, vol. 29, no. 12, p. 6443, 1984.

[27] S. Lynch, "A commentary on mechanisms of environmentally assisted cracking," Institute of Materials, vol. 684, pp. 206-219, 1997.

[28] Y. Tateyama and T. Ohno, "Stability and clusterization of hydrogen-vacancy complexes in $\alpha$ - fe: An ab initio study," Physical Review B, vol. 67, no. 17, p. 174105, 2003.

[29] K. Takai, H. Shoda, H. Suzuki, and M. Nagumo, "Lattice defects dominating hydrogen-related failure of metals," Acta Materialia, vol. 56, no. 18, pp. 51585167, 2008.

[30] S. Lynch, "A fractographic study of hydrogen-assisted cracking and liquid-metal embrittlement in nickel," Journal of Materials science, vol. 21, no. 2, pp. 692$704,1986$.

[31] F. H. Stillinger and T. A. Weber, "Dynamics of structural transitions in liquids," Physical Review A, vol. 28, no. 4, p. 2408, 1983.

[32] F. H. Stillinger and T. A. Weber, "Hidden structure in liquids," Physical Review $A$, vol. 25, no. 2, p. 978, 1982.

[33] F. H. Stillinger and T. A. Weber, "Packing structures and transitions in liquids and solids," Science(Washington, DC), vol. 225, no. 4666, pp. 983-9, 1984.

[34] F. H. Stillinger and T. A. Weber, "Computer simulation of local order in condensed phases of silicon," Physical review B, vol. 31, no. 8, p. 5262, 1985. 
[35] V. Shastry and D. Farkas, "Molecular statics simulation of fracture in-iron," Modelling and Simulation in Materials Science and Engineering, vol. 4, no. 5, p. $473,1996$.

[36] B. Wirth, G. Odette, D. Maroudas, and G. Lucas, "Energetics of formation and migration of self-interstitials and self-interstitial clusters in $\alpha$-iron," Journal of nuclear materials, vol. 244, no. 3, pp. 185-194, 1997.

[37] T. Parthasarathy, S. Rao, and D. Dimiduk, "Molecular statics simulations of core structures and motion of dislocations in nial," Philosophical Magazine A, vol. 67, no. 3, pp. 643-662, 1993.

[38] J. Nocedal and S. J. Wright, "Springer series in operations research. numerical optimization," 1999.

[39] T. Schlick, Molecular modeling and simulation: an interdisciplinary guide: an interdisciplinary guide, vol. 21. Springer Science \& Business Media, 2010.

[40] E. Bitzek, P. Koskinen, F. Ghler, M. Moseler, and P. Gumbsch, "Structural relaxation made simple," Physical review letters, vol. 97, no. 17, p. 170201, 2006 .

[41] D. Bachurin and P. Gumbsch, "Accommodation processes during deformation of nanocrystalline palladium," Acta Materialia, vol. 58, no. 16, pp. 5491-5501, 2010 .

[42] Z. Chen, M. Mrovec, and P. Gumbsch, "Atomistic aspects of screw dislocation behavior in $\alpha$-iron and the derivation of microscopic yield criterion," Modelling and Simulation in Materials Science and Engineering, vol. 21, no. 5, p. 055023, 2013.

[43] Z. Chen, M. Mrovec, and P. Gumbsch, "Dislocation-vacancy interactions in tungsten," Modelling and Simulation in Materials Science and Engineering, vol. 19, no. 7, p. 074002, 2011.

[44] T. Graham, "On the occlusion of hydrogen gas by metals," Proceedings of the Royal Society of London, vol. 16, pp. 422-427, 1867.

[45] N. F. Mott and H. Jones, The theory of the properties of metals and alloys. Courier Corporation, 1958. 
[46] G. Alefeld, J. Vlkl, and S. O. service), Hydrogen in Metals II: ApplicationOriented Properties, vol. 29.;29;. Berlin, Heidelberg: Springer Berlin Heidelberg, 1978.

[47] J. W. Haus and K. W. Kehr, "Diffusion in regular and disordered lattices," Physics Reports, vol. 150, no. 5, pp. 263-406, 1987.

[48] R. Oriani, "The physical and metallurgical aspects of hydrogen in metals," Fusion Technology, vol. 26, no. 4, pp. 235-266, 1994.

[49] W. Zhong, Y. Cai, D. Toma, et al., "Mechanical stability of pd-h systems: A molecular-dynamics study," Physical Review B, vol. 46, no. 13, p. 8099, 1992.

[50] R. Messmer and C. Briant, "The role of chemical bonding in grain boundary embrittlement," Acta Metallurgica, vol. 30, no. 2, pp. 457-467, 1982.

[51] R. Kirchheim, "Interaction of hydrogen with external stress fields," Acta Metallurgica, vol. 34, no. 1, pp. 37-42, 1986.

[52] J. Mao and R. McLellan, "The thermodynamics of hydrogen-vacancy interactions in aluminum," Journal of Physics and Chemistry of Solids, vol. 63, no. 11, pp. 2029-2036, 2002.

[53] S. M. Myers, M. Baskes, H. Birnbaum, J. W. Corbett, G. DeLeo, S. Estreicher, E. E. Haller, P. Jena, N. M. Johnson, R. Kirchheim, et al., "Hydrogen interactions with defects in crystalline solids," Reviews of Modern Physics, vol. 64, no. 2, p. 559, 1992.

[54] Y. Fukai, "Superabundant vacancies formed in metal-hydrogen alloys," Physica Scripta, vol. 2003, no. T103, p. 11, 2003.

[55] C. Budin, A. Lucasson, and P. Lucasson, "Études isochrones du cuivre trempé en atmosphère d'hydrogène," Journal de Physique, vol. 25, no. 7, pp. 751-756, 1964.

[56] G. Gaskey and R. Derrick, "Trapping of deuterium during permeation through gold," Scripta Metallurgica, vol. 10, no. 5, pp. 377-380, 1976.

[57] M. Nagumo, M. Nakamura, and K. Takai, "Hydrogen thermal desorption relevant to delayed-fracture susceptibility of high-strength steels," Metallurgical and Materials Transactions A, vol. 32, no. 2, pp. 339-347, 2001. 
[58] H. Birnbaum and P. Sofronis, "Mechanics of the hydrogen-dislocation-impurity interactions-increasing shear modulus j," Mech. Phys. Solids, vol. 43, pp. 49-90, 1995.

[59] S. Taketomi, R. Matsumoto, and N. Miyazaki, "Atomistic simulation of the effects of hydrogen on the mobility of edge dislocation in alpha iron," Journal of Materials Science, vol. 43, no. 3, pp. 1166-1169, 2008.

[60] Y. Fukai, M. Mizutani, S. Yokota, M. Kanazawa, Y. Miura, and T. Watanabe, "Superabundant vacancy-hydrogen clusters in electrodeposited ni and cu," Journal of alloys and compounds, vol. 356, pp. 270-273, 2003.

[61] D. Tanguy, Y. Wang, and D. Connétable, "Stability of vacancy-hydrogen clusters in nickel from first-principles calculations," Acta Materialia, vol. 78, pp. 135-143, 2014.

[62] D. Connétable, Y. Wang, and D. Tanguy, "Segregation of hydrogen to defects in nickel using first-principles calculations: The case of self-interstitials and cavities," Journal of Alloys and Compounds, vol. 614, pp. 211-220, 2014.

[63] Y. Fukai and N. Ōkuma, "Evidence of copious vacancy formation in ni and pd under a high hydrogen pressure," Japanese journal of applied physics, vol. 32, no. 9A, p. L1256, 1993.

[64] M. Iwamoto and Y. Fukai, "Superabundant vacancy formation in iron under high hydrogen pressures - thermal desorption spectroscopy," JIM, Materials Transactions, vol. 40, no. 7, pp. 606-611, 1999.

[65] Y. Shirai, H. Araki, T. Mori, W. Nakamura, and K. Sakaki, "Positron annihilation study of lattice defects induced by hydrogen absorption in some hydrogen storage materials," Journal of alloys and compounds, vol. 330, pp. 125-131, 2002 .

[66] K. Wilson and M. Baskes, "Deuterium trapping in irradiated 316 stainless steel," Journal of Nuclear Materials, vol. 76, pp. 291-297, 1978.

[67] R. G. Hennig, D. R. Trinkle, J. Bouchet, S. G. Srinivasan, R. C. Albers, and J. W. Wilkins, "Impurities block the $\alpha$ to $\omega$ martensitic transformation in titanium," Nature materials, vol. 4, no. 2, pp. 129-133, 2005.

[68] D. Westlake, "A geometric rationale for hydrogenated pd 3 p 1- x," Journal of the Less Common Metals, vol. 107, no. 2, pp. 189-202, 1985. 
[69] I. Jacob, O. Beeri, and E. Elish, "Interstitial sites in the hydrogen-inactive intermetallic compounds zrpd 2 and upd 3," Journal of alloys and compounds, vol. 221, no. 1, pp. 129-132, 1995.

[70] C. Jiang, S. Maloy, and S. Srinivasan, "A computational method to identify interstitial sites in complex materials," Scripta Materialia, vol. 58, no. 9, pp. 739 742, 2008.

[71] Y. Tang and J. A. El-Awady, "Atomistic simulations of the interactions of hydrogen with dislocations in fcc metals," Physical Review B, vol. 86, no. 17, p. 174102, 2012.

[72] D. Hull and D. J. Bacon, Introduction to dislocations, vol. 257. Pergamon Press Oxford, 1984.

[73] R. Oriani, "Hydrogen embrittlement of steels," Annual review of materials science, vol. 8, no. 1, pp. 327-357, 1978.

[74] C. Beachem, "A new model for hydrogen-assisted cracking (hydrogen embrittlement)," Metallurgical transactions, vol. 3, no. 2, pp. 441-455, 1972.

[75] J. P. Hirth, "Effects of hydrogen on the properties of iron and steel," Metallurgical Transactions A, vol. 11, no. 6, pp. 861-890, 1980.

[76] M. Nagumo, "Hydrogen related failure of steels-a new aspect," Materials Science and Technology, vol. 20, no. 8, pp. 940-950, 2004.

[77] A. R. Troiano, "The role of hydrogen and other interstitials in the mechanical behavior of metals," trans. ASM, vol. 52, no. 1, pp. 54-80, 1960.

[78] A. Tetelman and W. Robertson, "The mechanism of hydrogen embrittlement observed in iron-silicon single crystals," tech. rep., DTIC Document, 1961.

[79] S. Gahr, M. Grossbeck, and H. Birnbaum, "Hydrogen embrittlement of nb imacroscopic behavior at low temperatures," Acta Metallurgica, vol. 25, no. 2, pp. 125-134, 1977.

[80] I. Robertson, "The effect of hydrogen on dislocation dynamics," Engineering Fracture Mechanics, vol. 68, no. 6, pp. 671-692, 2001.

[81] H. K. Birnbaum, "Hydrogen effects on deformation and fracture: Science and sociology," MRS Bulletin, vol. 28, no. 7, pp. 479-485, 2003. 
[82] R. P. Gangloff and B. P. Somerday, Gaseous Hydrogen Embrittlement of Materials in Energy Technologies, vol. 1. Woodhead Publishing, 2012.

[83] A. Kimura and H. K. Birnbaum, "Hydrogen induced grain boundary fracture in high purity nickel and its alloysenhanced hydrogen diffusion along grain boundaries," Acta Metallurgica, vol. 36, no. 3, pp. 757-766, 1988.

[84] H. K. Birnbaum and P. Sofronis, "Hydrogen-enhanced localized plasticitya mechanism for hydrogen-related fracture," Materials Science 8 Engineering A, vol. 176, no. 1, pp. 191-202, 1994.

[85] S. Asano and R. Otsuka, "The lattice hardening due to dissolved hydrogen in iron and steel," Scripta Metallurgica, vol. 10, no. 11, pp. 1015-1020, 1976.

[86] T. Marrow, C. Hippsley, and J. King, "Effect of mean stress on hydrogen assisted fatique crack propagation in duplex stainless steel," Acta metallurgica et materialia, vol. 39, no. 6, pp. 1367-1376, 1991.

[87] D. P. Abraham and C. J. Altstetter, "The effect of hydrogen on the yield and flow stress of an austenitic stainless steel," Metallurgical and Materials transactions $A$, vol. 26, no. 11, pp. 2849-2858, 1995.

[88] Y. Murakami, T. Kanezaki, and Y. Mine, "Hydrogen effect against hydrogen embrittlement," Metallurgical and Materials Transactions A, vol. 41, no. 10, pp. 2548-2562, 2010.

[89] M. S. Daw, M. Baskes, C. Bisson, and W. Wolfer, "Application of the embedded atom method to fracture, dislocation dynamics, and hydrogen embrittlement.," in Metallurgical Soc of AIME, 1986.

[90] J. Song and W. Curtin, "A nanoscale mechanism of hydrogen embrittlement in metals," acta materialia, vol. 59, no. 4, pp. 1557-1569, 2011.

[91] M. Wen, S. Fukuyama, and K. Yokogawa, "Atomistic simulations of effect of hydrogen on kink-pair energetics of screw dislocations in bcc iron," Acta materialia, vol. 51, no. 6, pp. 1767-1773, 2003.

[92] G. Lu, D. Orlikowski, I. Park, O. Politano, and E. Kaxiras, "Energetics of hydrogen impurities in aluminum and their effect on mechanical properties," Physical Review B, vol. 65, no. 6, p. 064102, 2002. 
[93] G. Pressouyre, "Trap theory of hydrogen embrittlement," Acta Metallurgica, vol. 28, no. 7, pp. 895-911, 1980.

[94] S. Picraux, "Defect trapping of gas atoms in metals," Nuclear Instruments and Methods, vol. 182, pp. 413-437, 1981.

[95] F. Besenbacher, S. Myers, P. Nordlander, J. No, et al., "Multiple hydrogen occupancy of vacancies in fe," Journal of applied physics, vol. 61, no. 5, pp. 1788$1794,1987$.

[96] K. Schmid, U. von Toussaint, and T. Schwarz-Selinger, "Transport of hydrogen in metals with occupancy dependent trap energies," Journal of Applied Physics, vol. 116, no. 13, p. 134901, 2014.

[97] S. Myers, P. Nordlander, F. Besenbacher, J. No, et al., "Theoretical examination of the trapping of ion-implanted hydrogen in metals," Physical Review B, vol. 33, no. 2, p. 854, 1986.

[98] F. Besenbacher, B. B. Nielsen, J. Nørskov, S. Myers, and P. Nordlander, "Interaction of hydrogen isotopes with metals: Deuterium trapped at lattice defects in palladium," Journal of Fusion Energy, vol. 9, no. 3, pp. 257-261, 1990.

[99] F. Besenbacher, H. Bogh, A. Pisarev, M. Puska, S. Holloway, and J. Nørskov, "Interaction of deuterium with lattice defects in nickel," Nuclear Instruments and Methods in Physics Research Section B: Beam Interactions with Materials and Atoms, vol. 4, no. 3, pp. 374-387, 1984.

[100] F. Besenbacher, J. Bo, S. Myers, et al., "Defect trapping of ion-implanted deuterium in nickel," Journal of Applied Physics, vol. 53, no. 5, pp. 3536-3546, 1982.

[101] W.-S. Ko, J. B. Jeon, J.-H. Shim, and B.-J. Lee, "Origin of hydrogen embrittlement in vanadium-based hydrogen separation membranes," international journal of hydrogen energy, vol. 37, no. 18, pp. 13583-13593, 2012.

[102] L. S. Darken and R. P. Smith, "Behavior of hydrogen in steel during and after immersion in acid," Corrosion, vol. 5, no. 1, pp. 1-16, 1949.

[103] J. Takahashi, K. Kawakami, Y. Kobayashi, and T. Tarui, "The first direct observation of hydrogen trapping sites in tic precipitation-hardening steel through atom probe tomography," Scripta Materialia, vol. 63, no. 3, pp. 261-264, 2010. 
[104] J. Takahashi, K. Kawakami, and T. Tarui, "Direct observation of hydrogentrapping sites in vanadium carbide precipitation steel by atom probe tomography," Scripta Materialia, vol. 67, no. 2, pp. 213-216, 2012.

[105] M. Christensen, W. Wolf, C. Freeman, E. Wimmer, R. Adamson, L. Hallstadius, P. Cantonwine, and E. Mader, "Effect of hydrogen on dimensional changes of zirconium and the influence of alloying elements: First-principles and classical simulations of point defects, dislocation loops, and hydrides," in Zirconium in the Nuclear Industry: 17th Volume, ASTM International, 2015.

[106] P. Ferreira, I. Robertson, and H. Birnbaum, "Hydrogen effects on the interaction between dislocations," Acta materialia, vol. 46, no. 5, pp. 1749-1757, 1998.

[107] P. Ferreira, I. Robertson, and H. Birnbaum, "Hydrogen effects on the character of dislocations in high-purity aluminum," Acta materialia, vol. 47, no. 10, pp. 2991-2998, 1999.

[108] A. H. Cottrell, Dislocations and plastic flow in crystals. Clarendon Press, 1965.

[109] I. Salehinia and S. Medyanik, "Effects of vacancies on the onset of plasticity in metalsan atomistic simulation study," Metallurgical and Materials Transactions A, vol. 42, no. 13, pp. 3868-3874, 2011.

[110] N. Mousseau and G. T. Barkema, "Exploring high-dimensional energy landscapes," Computing in Science \& Engineering, vol. 1, no. 2, pp. 74-80, 1999.

[111] N. Mousseau and G. T. Barkema, "Event-based relaxation of continuous disordered systems," Physical review letters, vol. 77, no. 21, pp. 4358-4361, 1996.

[112] R. Malek and N. Mousseau, "Dynamics of lennard-jones clusters: A characterization of the activation-relaxation technique," Physical Review E, vol. 62, no. 6, p. $7723,2000$.

[113] F. El-Mellouhi, N. Mousseau, and L. Lewis, "Kinetic activation-relaxation technique: An off-lattice self-learning kinetic monte carlo algorithm," PHYSICAL REVIEW B, vol. 78, no. 15, 2008.

[114] L. K. Béland, P. Brommer, F. El-Mellouhi, J.-F. Joly, and N. Mousseau, "Kinetic activation-relaxation technique," Physical Review E, vol. 84, no. 4, p. 046704, 2011. 
[115] J.-F. Joly, L. K. Béland, P. Brommer, F. El-Mellouhi, and N. Mousseau, "Optimization of the kinetic activation-relaxation technique, an off-lattice and selflearning kinetic monte-carlo method," in Journal of Physics: Conference Series, vol. 341, p. 012007, IOP Publishing, 2012.

[116] M.-C. Marinica, F. Willaime, and N. Mousseau, "Energy landscape of small clusters of self-interstitial dumbbells in iron," Physical Review B, vol. 83, no. 9, p. 094119, 2011.

[117] E. Machado-Charry, L. K. Béland, D. Caliste, L. Genovese, T. Deutsch, N. Mousseau, and P. Pochet, "Optimized energy landscape exploration using the ab initio based activation-relaxation technique," The Journal of chemical physics, vol. 135, no. 3, p. 034102, 2011.

[118] K. Z. Rushchanskii, P. Pochet, and F. Lançon, "Stress enhanced self-diffusion in si: Entropy effect in anisotropic elastic environment," Applied Physics Letters, vol. 92, no. 15, p. 152110, 2008.

[119] D. Caliste, K. Z. Rushchanskii, and P. Pochet, "Vacancy-mediated diffusion in biaxially strained si," Applied Physics Letters, vol. 98, no. 3, p. 031908, 2011.

[120] N. Mousseau, L. K. Béland, P. Brommer, F. El-Mellouhi, J.-F. Joly, G. K. NTsouaglo, O. Restrepo, and M. Trochet, "Following atomistic kinetics on experimental timescales with the kinetic activation-relaxation technique," Computational Materials Science, vol. 100, pp. 111-123, 2015.

[121] O. A. Restrepo, N. Mousseau, F. El-Mellouhi, O. Bouhali, M. Trochet, and C. S. Becquart, "Diffusion properties of fe-c systems studied by using kinetic activation-relaxation technique," Computational Materials Science, vol. 112, pp. 96-106, 2016.

[122] S. Plimpton, "Fast parallel algorithms for short-range molecular dynamics," Journal of Computational Physics, vol. 117, no. 1, pp. 1-19, 1995.

[123] "Lammps www site." lammps.sandia.gov/index.html. Accessed: 2014-10-03.

[124] A. Stukowski, "Visualization and analysis of atomistic simulation data with ovito-the open visualization tool," Modelling and Simulation in Materials Science and Engineering, vol. 18, no. 1, p. 015012, 2009.

[125] J. E. Lennard-Jones, "Cohesion," Proceedings of the Physical Society, vol. 43, no. 5, p. 461, 1931. 
[126] X. Liu, J. Wang, and S. Biner, "Hydrogen and self-interstitial interactions with edge dislocations in ni: atomistic and elasticity comparisons," Modelling and Simulation in Materials Science and Engineering, vol. 16, no. 4, p. 045002, 2008.

[127] "Openkim knowledgebase of interatomic models www site." https://openkim. org/intro-models//. Accessed: 2014-10-03.

[128] F. Birch, "Finite elastic strain of cubic crystals," Physical Review, vol. 71, no. 11, p. 809, 1947.

[129] E. Wimmer, W. Wolf, J. Sticht, P. Saxe, C. B. Geller, R. Najafabadi, and G. A. Young, "Temperature-dependent diffusion coefficients from ab initio computations: Hydrogen, deuterium, and tritium in nickel," Physical Review B, vol. 77, no. 13, p. 134305, 2008.

[130] C. Barrett, "Tb massalski structure of metals," 1966.

[131] W. F. Gale and T. C. Totemeier, Smithells metals reference book. ButterworthHeinemann, 2003.

[132] W. Wycisk and M. Feller-Kniepmeier, "Quenching experiments in high purity ni," Journal of Nuclear Materials, vol. 69, pp. 616-619, 1978. 
Appendix A

Hydrogen trapping energy as a function of system size 


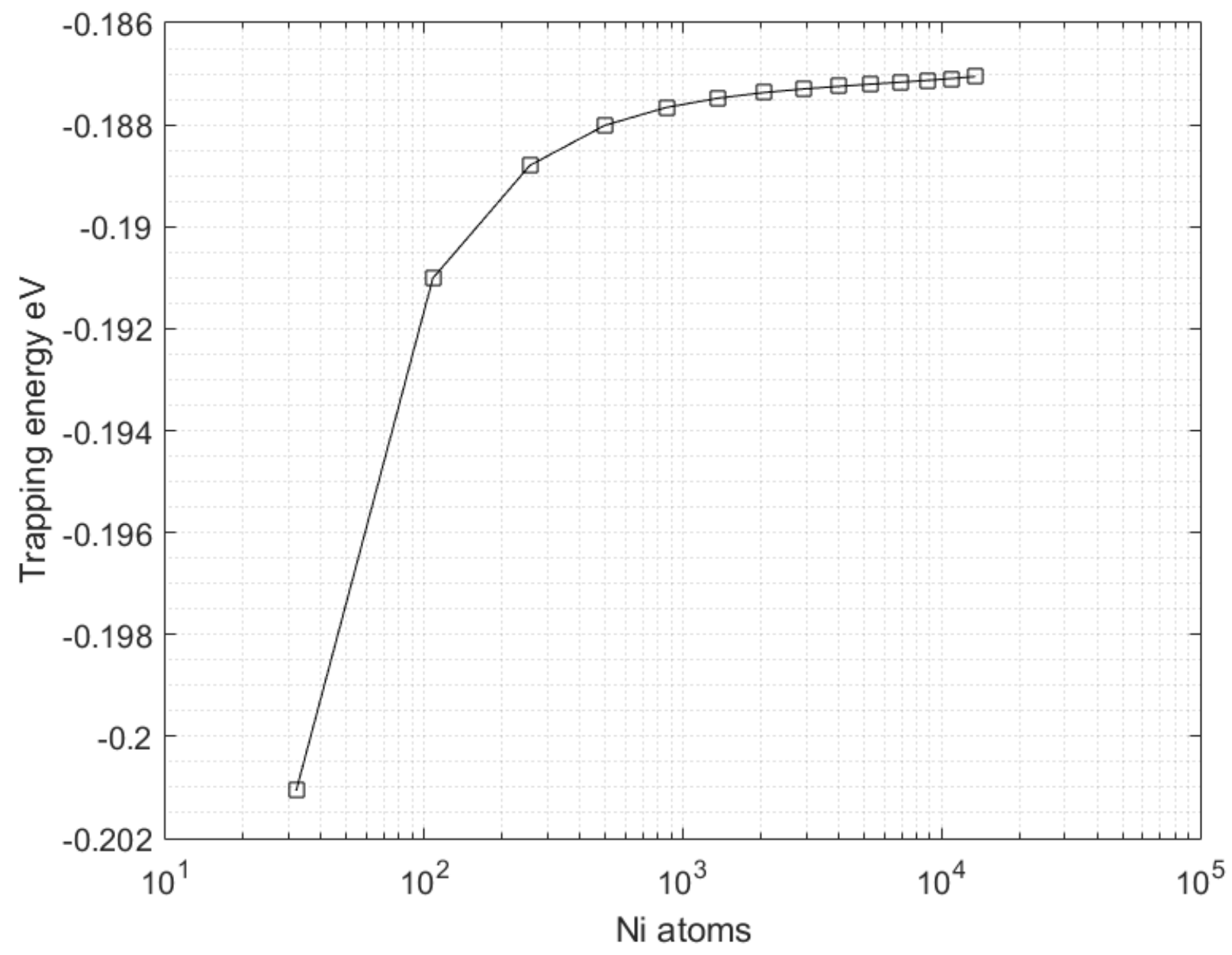

Figure A.1: The trapping energy of 1 hydrogen atom as a function of system size 


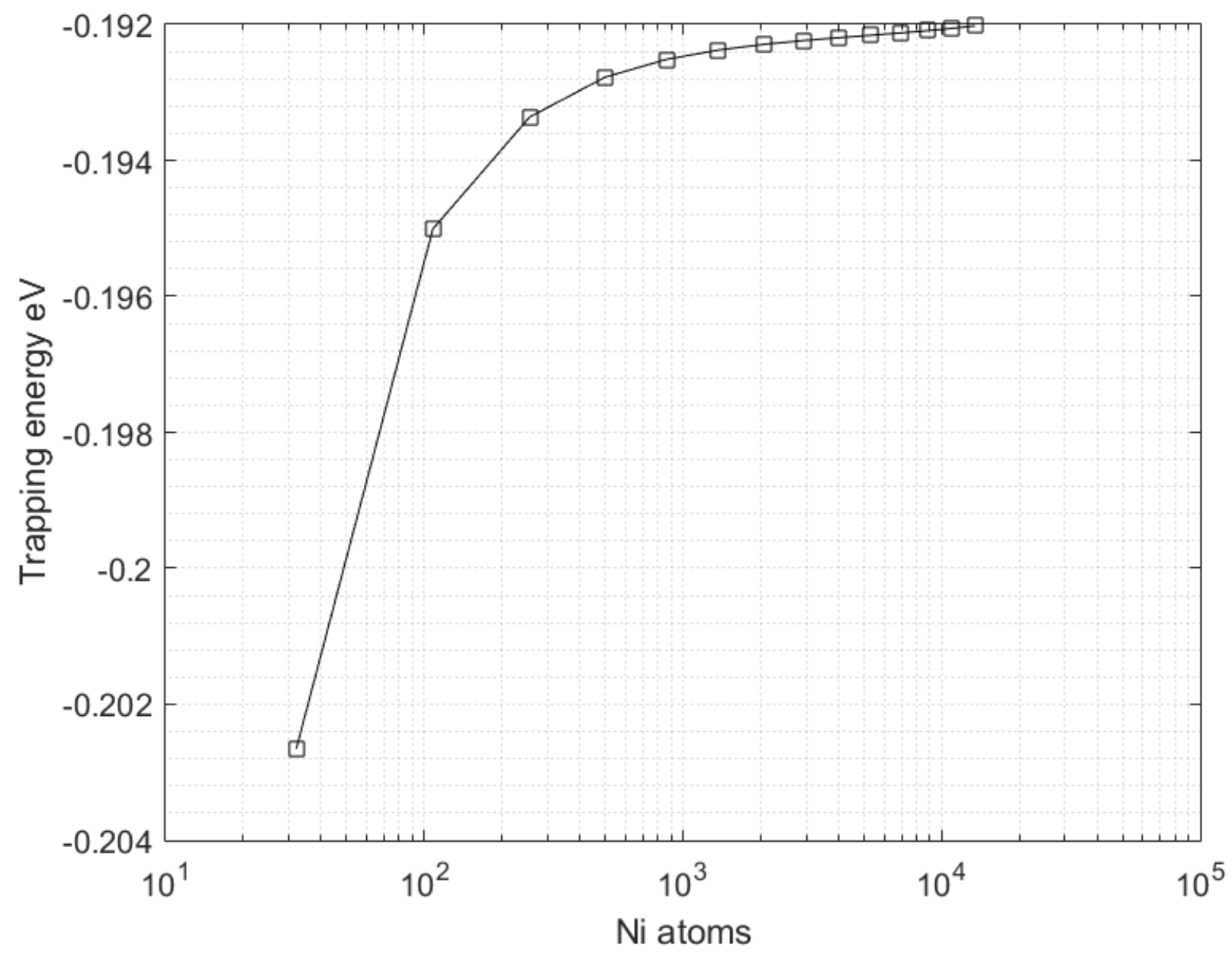

Figure A.2: The trapping energy of 2 hydrogen atoms as a function of system size 


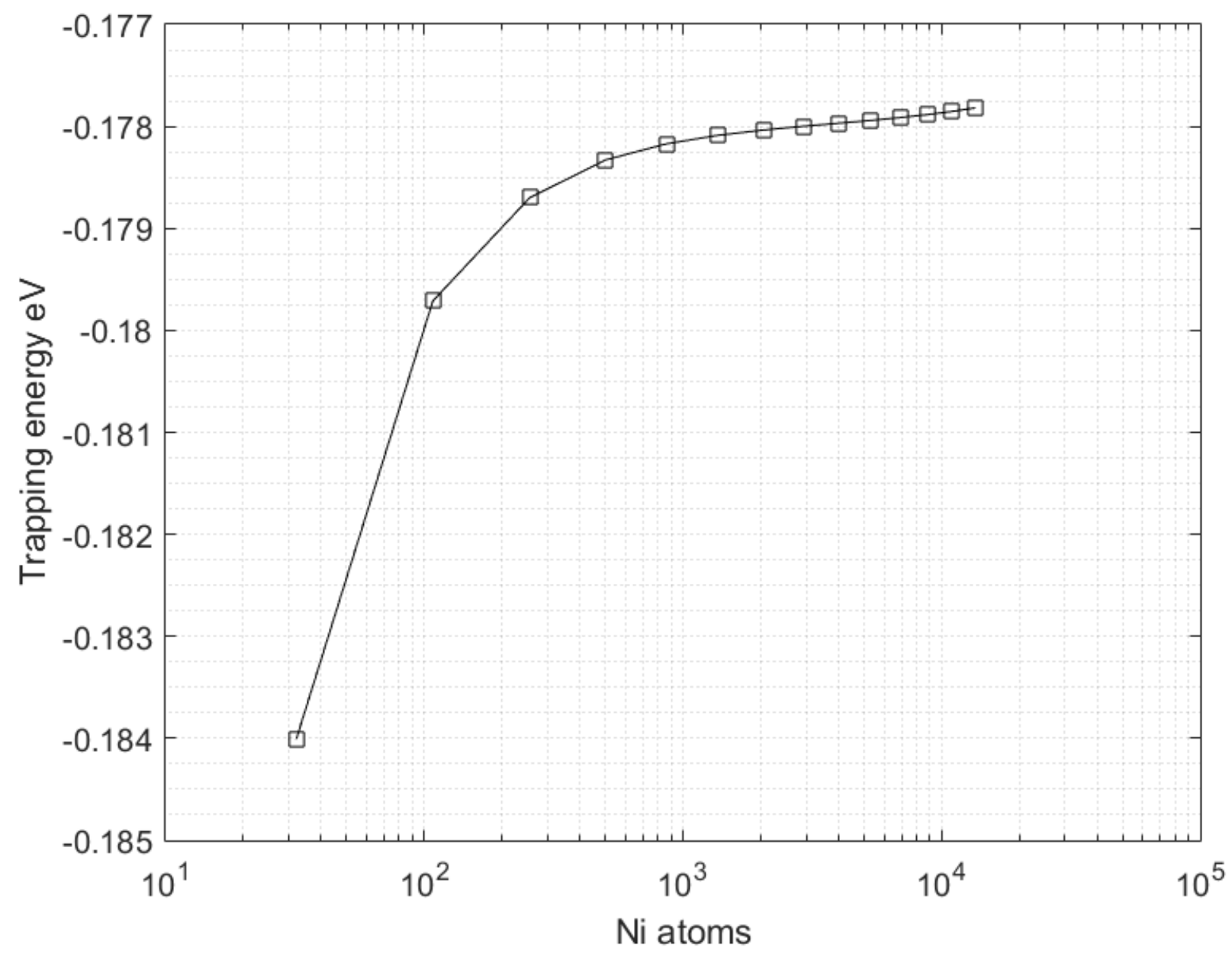

Figure A.3: The trapping energy of 3 hydrogen atoms as a function of system size 


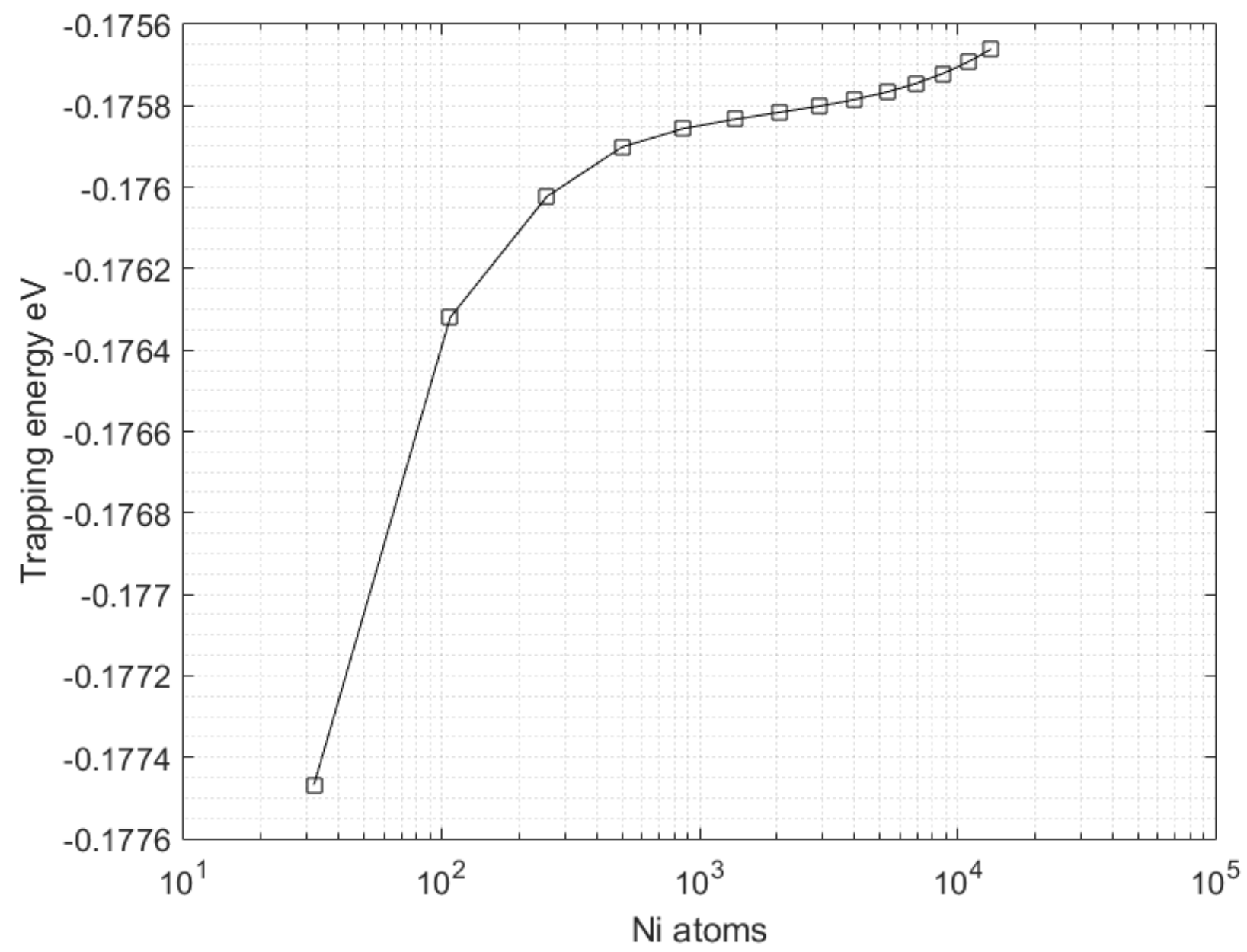

Figure A.4: The trapping energy of 4 hydrogen atoms as a function of system size 


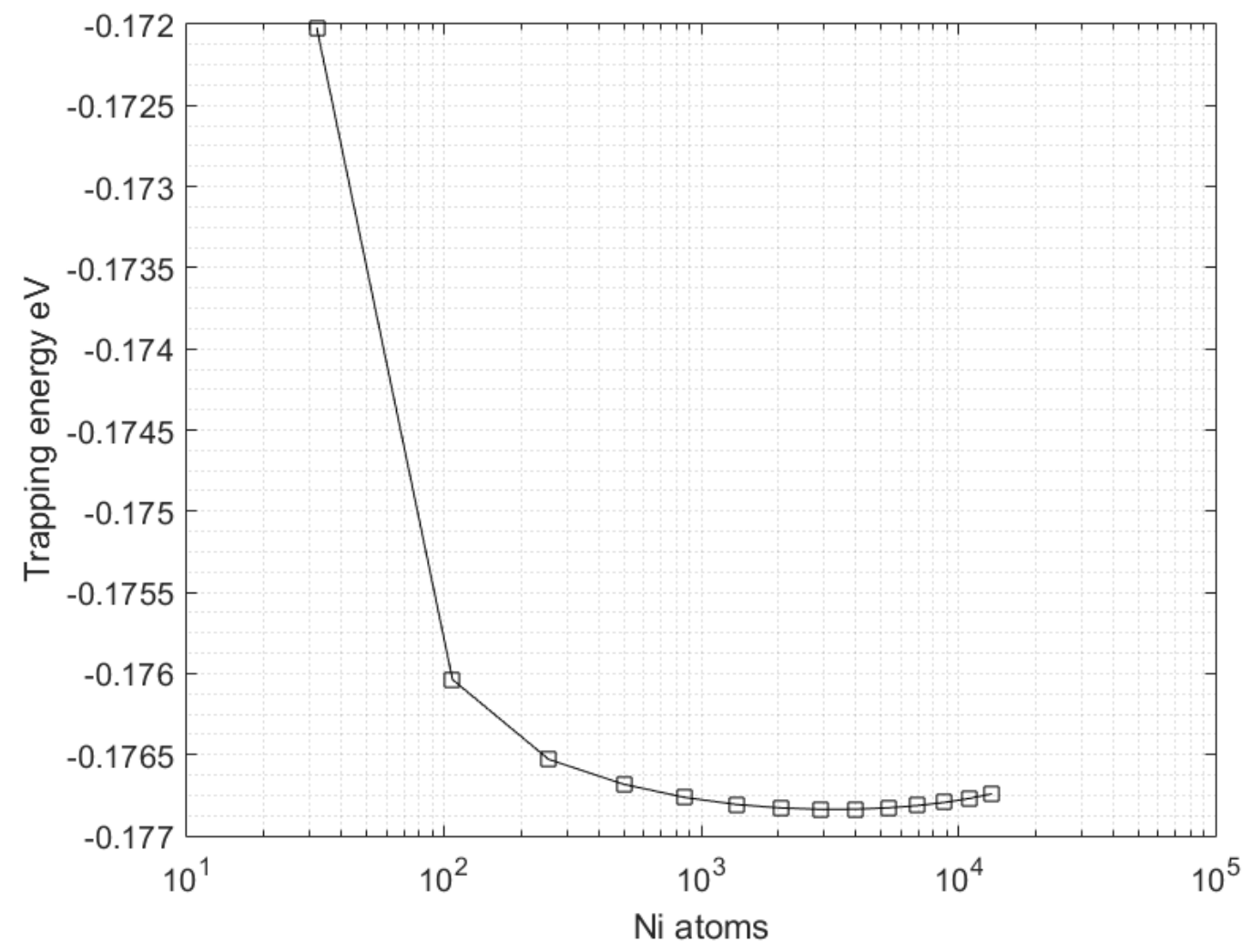

Figure A.5: The trapping energy of 5 hydrogen atoms as a function of system size 


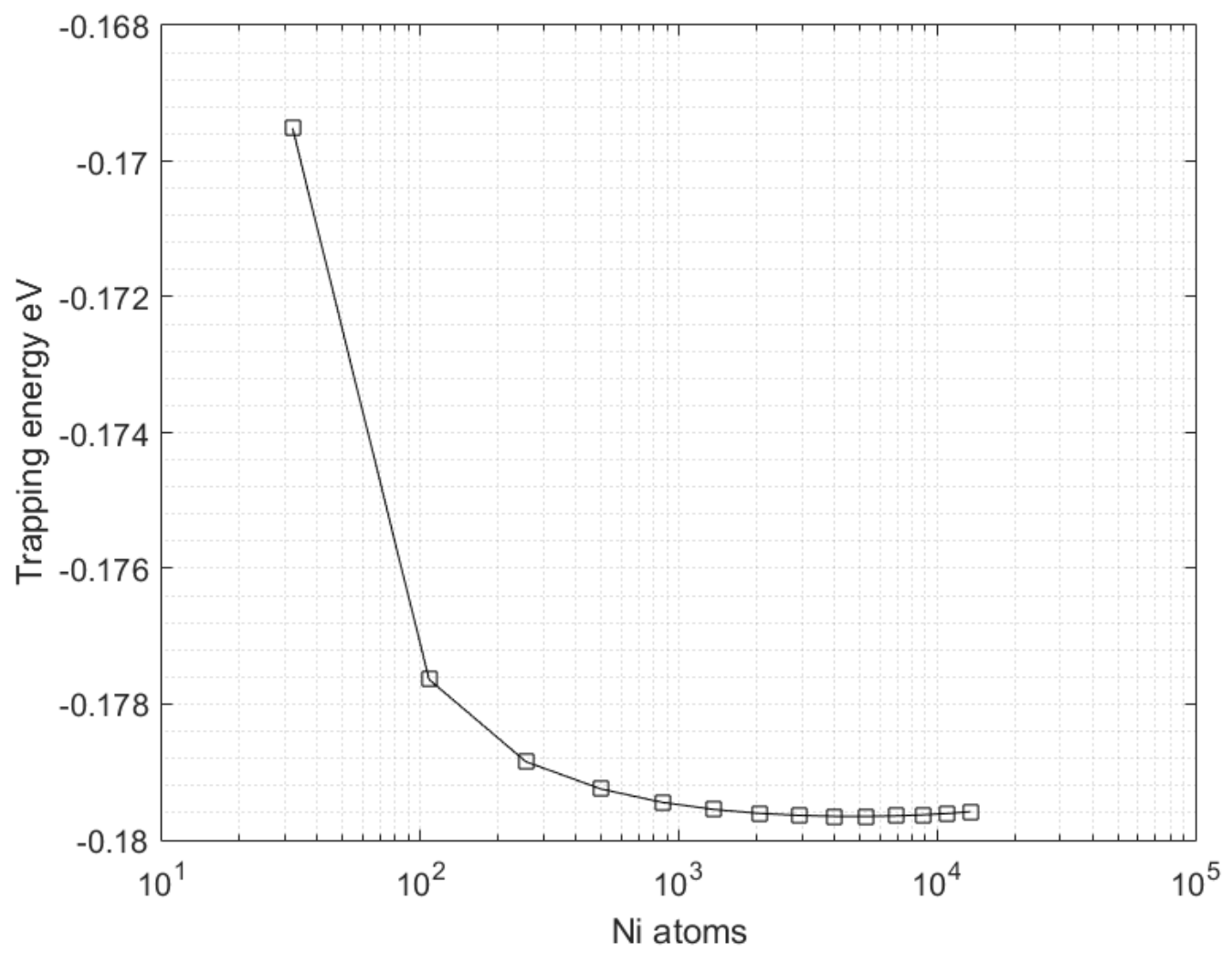

Figure A.6: The trapping energy of 6 hydrogen atoms as a function of system size 


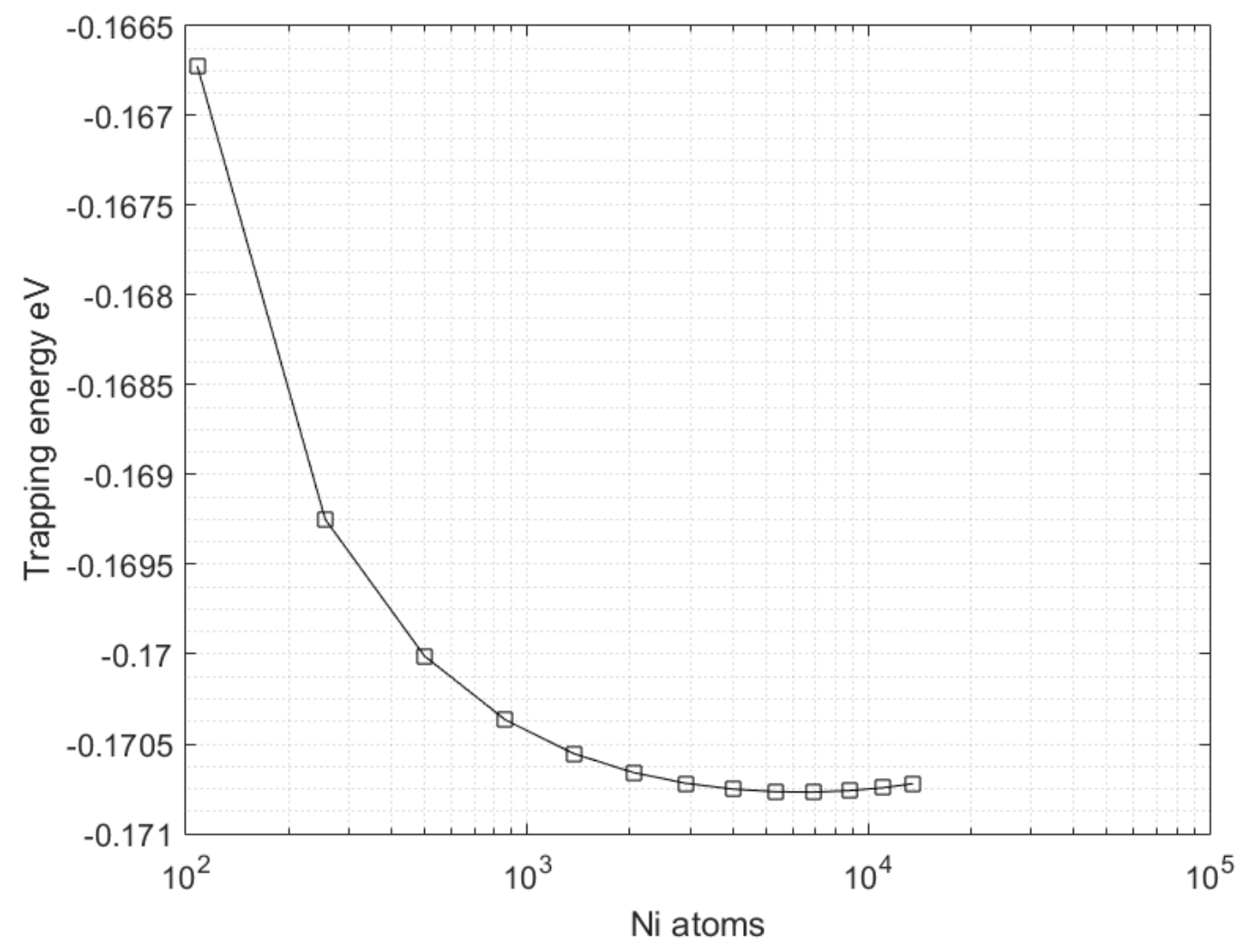

Figure A.7: The trapping energy of 7 hydrogen atoms as a function of system size 


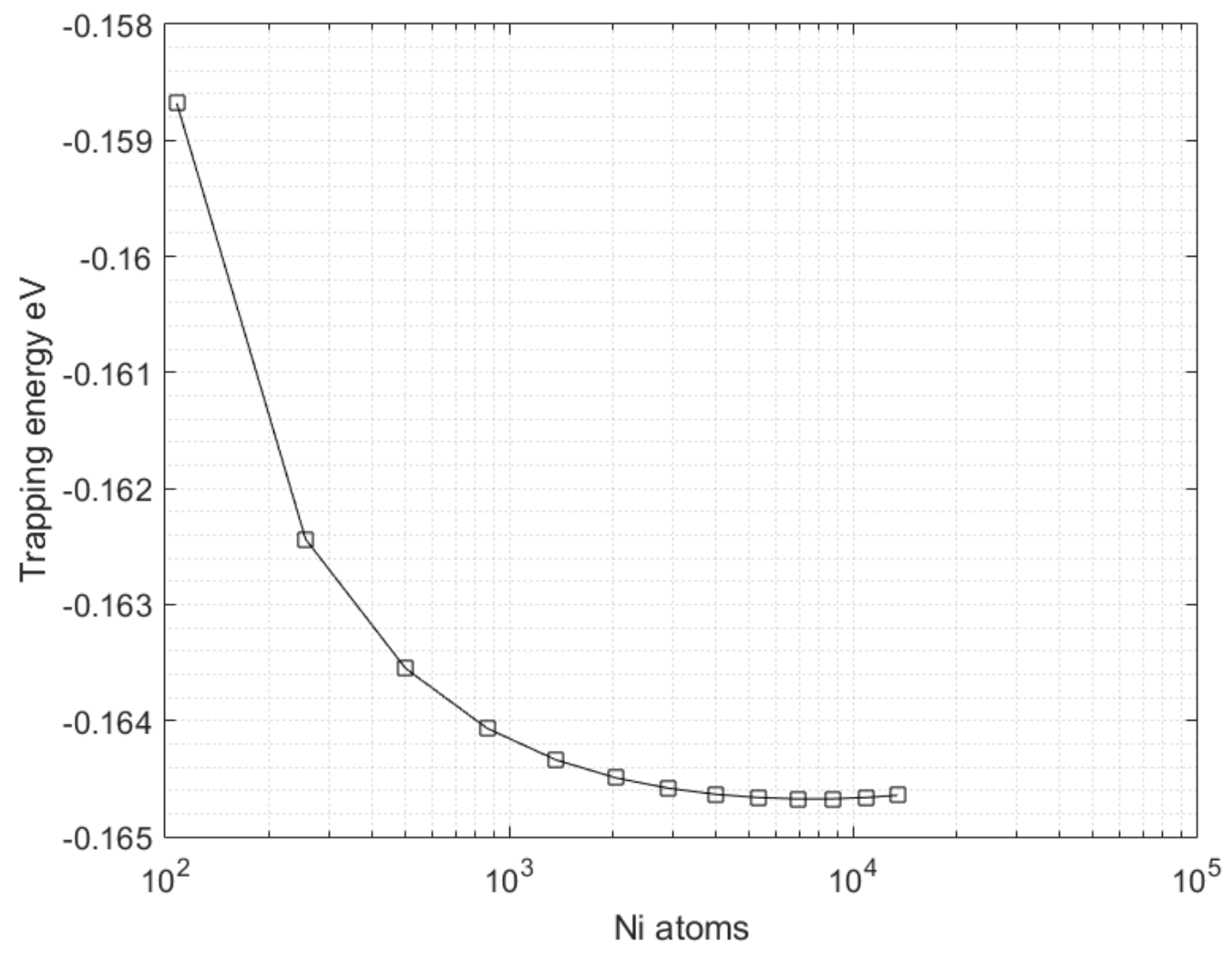

Figure A.8: The trapping energy of 8 hydrogen atoms as a function of system size 


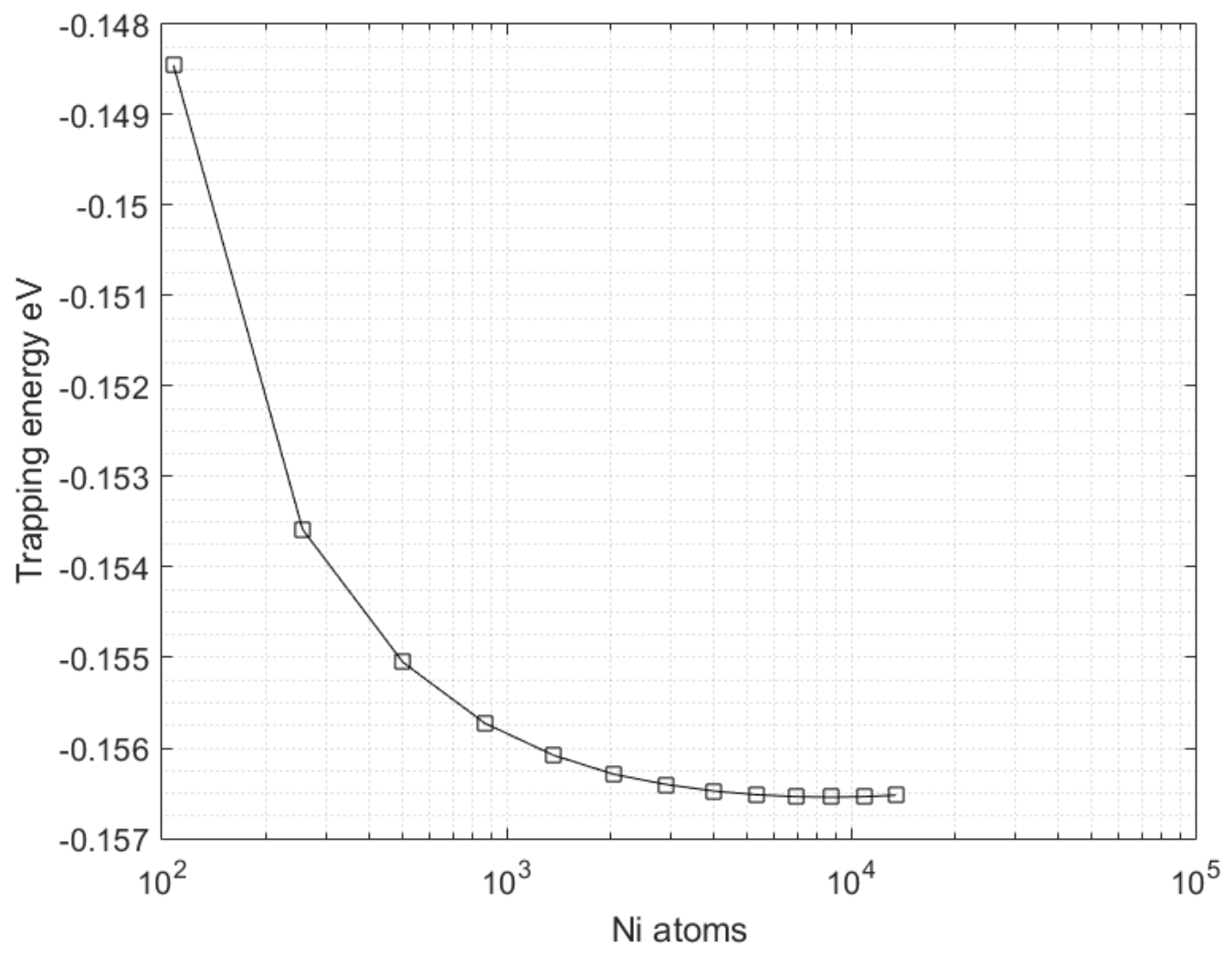

Figure A.9: The trapping energy of 9 hydrogen atoms as a function of system size 


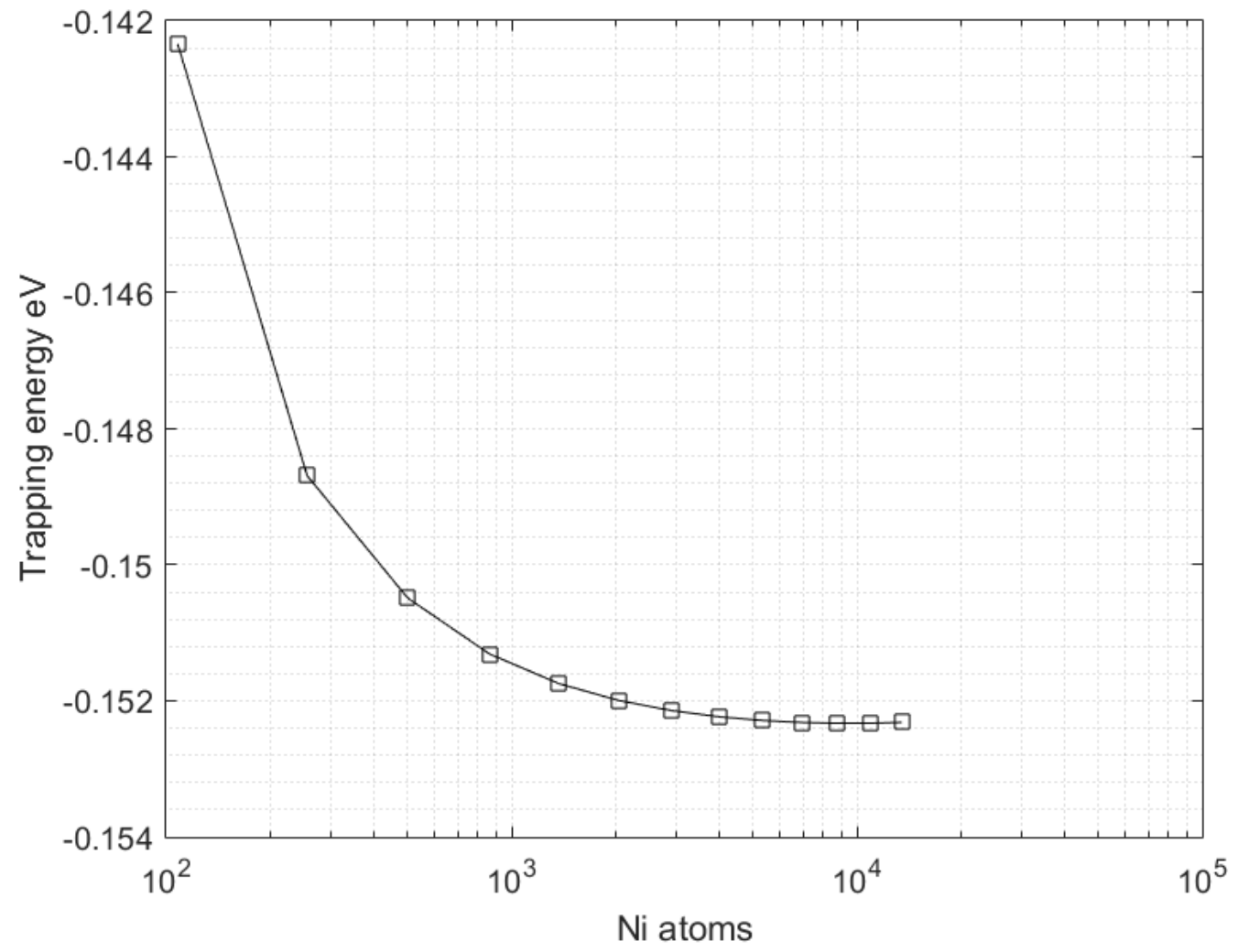

Figure A.10: The trapping energy of 10 hydrogen atoms as a function of system size 


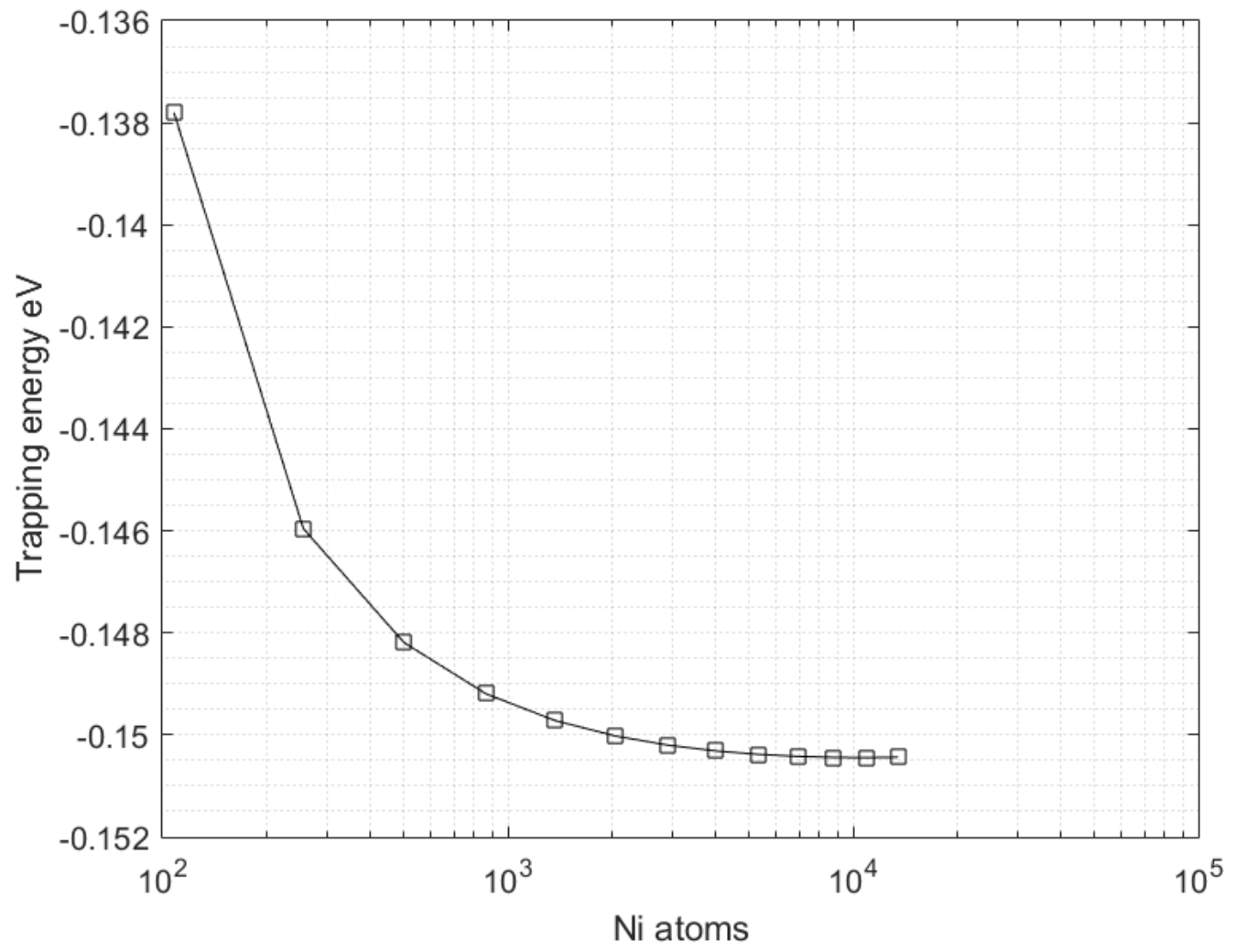

Figure A.11: The trapping energy of 11 hydrogen atoms as a function of system size 


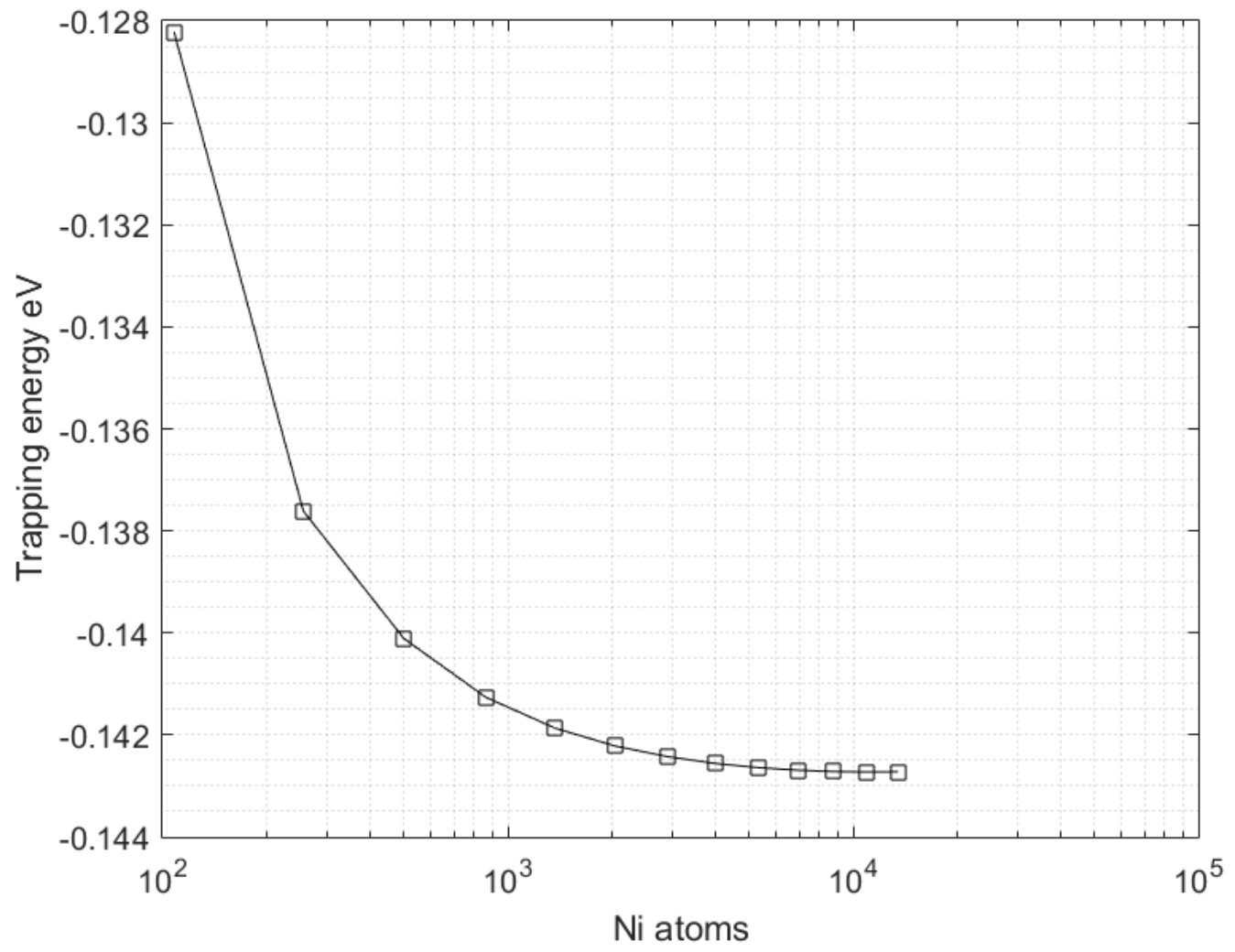

Figure A.12: The trapping energy of 12 hydrogen atoms as a function of system size 